\title{
Binary Galaxies in the Local Supercluster and Its Neighborhood
}

\author{
I. D. Karachentsev ${ }^{1}$ and D. I. Makarov ${ }^{1,2}$ \\ ${ }^{1}$ Special Astrophysical Observatory of the Russian AS, Nizhnij Arkhyz 369167, Russid* \\ ${ }^{2}$ Invited Researcher Observatoire de Lyon, St-Genis Laval Cedex, France 69561
}

(Received May 22, 2008; Revised June 9, 2008)

\begin{abstract}
We report a catalog of 509 pairs identified among 10403 nearby galaxies with lineof-sight velocities $V_{L G}<3500 \mathrm{~km} / \mathrm{s}$. We selected binary systems in accordance with two criteria ("bounding" and "temporal"), which require the physical pair of galaxies to have negative total energy and its components to be located inside the zerovelocity surface. We assume that individual galaxy masses are proportional to their total $K$-band luminosities, $M=L_{K} \times 6 M_{\odot} / L_{\odot}$. The catalog gives the magnitudes and morphological types of galaxies and also the projected (orbital) masses and pair isolation indices. The component line-of-sight velocity differences and projected distances of the binary systems considered have power-law distributions with the median values of $35 \mathrm{~km} / \mathrm{s}$ and $123 \mathrm{kpc}$, respectively. The median mass-to- $K$-band luminosity ratio is equal to $11 M_{\odot} / L_{\odot}$, and its uncertainty is mostly due to the errors of measured velocities. Our sample of binary systems has a typical density contrast of $\delta \rho / \rho_{c} \sim 500$ and a median crossing time of about 3.5 Gyr. We point out the substantial fraction of binary systems consisting of late-type dwarf galaxies, where the luminosities of both components are lower than that of the Small Magellanic Cloud. The median projected distance for 41 such pairs is only $30 \mathrm{kpc}$, and the median difference of their line-of-sight velocities is equal to $14 \mathrm{~km} / \mathrm{s}$ which is smaller than the typical error for radial-velocity $(30 \mathrm{~km} / \mathrm{s})$. This specific population of gasrich dwarf binary galaxies such as I Zw 18 may be at the stage immediately before merging of its components. Such objects, which are usually lost in flux-limited (and not distance-limited) samples deserve a thorough study in the $H I$ radio line with high spatial and velocity resolution.
\end{abstract}

\section{INTRODUCTION}

*Electronic address: ikar@luna.sao.ru

This is the first paper of a series devoted to the study of the visible and dark mat- 
ter within the nearby, but sufficiently representative volume of the Local Supercluster (LS) and its neighborhood, which is comparable to the size of a cosmological homogeneity cell. Over the past decade mass spectroscopic and photometric galaxy surveys-SloanDSS, 2MASS, $2 \mathrm{dF}$, and $6 \mathrm{dF}$ - have been performed, which reshaped and refined our concepts about the large-scale structure of the Universe. However, the surveys performed within certain sky areas or in narrow strips out to redshifts $z \simeq 0.1-1.0$ proved to be insufficient to allow analyses of the structure and kinematics of small- scale features like the Local Group, since they did not include numerous dwarf galaxies because of their low luminosity. For example, in the Sloan Digital Sky Survey (SDSS) the mean distance between galaxies with measured line-of-sight velocities is equal to $9 \mathrm{Mpc}$, which exceeds the diameter of a typical cluster.

In recent years, considerable effort was focused on the study of the most nearby, socalled Local Volume (LV) of radius $10 \mathrm{Mpc}$, where more than 500 galaxies have been found. Most of these objects are dwarf systems with measured line-of-sight velocities, and about half of these galaxies already have individual distance estimates that are accurate to at least $10 \%$, supported by observations made with the Hubble Space Telescope. The detailed 3D pattern of the distri- bution of galaxies in the LV, where the density of galaxies with measured velocities is two orders of magnitude higher than the corresponding density for the SDSS, 2dF, and $6 \mathrm{dF}$ surveys, allowed the structure and kinematics of groupings to be studied on scale lengths $0.1-1 \mathrm{Mpc}[1,2,2]$. The contribution of the virial masses of nearby groups proved to be three-to-four times less than the average cosmological density, $\Omega_{m}=0.27$. This inconsistency between the local and global $\Omega_{m}$ estimates can be due to poor statistics or to some specifics of our immediate neighborhood. An evident way for resolving this paradox consists in increasing the volume studied to make it include the entire Local supercluster and its immediate neighborhood. Tully [4, 5] was the first to successfully undertake such an analysis. He compiled a catalog and an atlas of nearby galaxies with line-of-sight velocities smaller than $3000 \mathrm{~km} / \mathrm{s}$. Tully's catalog contains a total of 2367 galaxies located inside the volume of diameter $82 \mathrm{Mpc}$, which is comparable to the volume of a cosmological homogeneity cell. Tully used the hierarchical dendrogram method to identify in this volume a total of 179 groups, which included $69 \%$ of all the galaxies considered. He then used the virial masses of these groups to infer a lower limit for the local mass density, $\Omega_{v i r}=0.08$, which proved to be three times smaller than the global value of $\Omega_{m}=0.27$. 
The amount of dark matter per unit luminosity of galaxies is known to increase from small groups to rich clusters. However, the virial regions of clusters contain only $5-10 \%$ of all galaxies, and about the same number of galaxies are associated with the unvirialized peripheral regions of these clusters. About half of all galaxies are members of groups like our Local Group, about one fourth of all galaxies reside in dispersed groups (clouds), and a total of $5-10 \%$ of all galaxies are located in the overall field. In such rather arbitrary and coarse partition groups of galaxies are the main contributors to the global mean mass density. However, the characteristic estimates of the masses of groups of galaxies differ by more than one order of magnitude. This circumstance emphasizes the need for further refinement of virial masses of groups of galaxies, which is great importance for cosmology.

Below we consider galaxies with line-ofsight velocities with respect to the Local Group $V_{L G}<3500 \mathrm{~km} / \mathrm{s}$. After excluding the region of strong absorption at Galactic latitudes $|b|<15^{\circ}$ we fixed a total of 10403 galaxies in this volume and applied to them the criterion of identifying multiple systems.

In this paper we consider only binary galaxies, because the corresponding sample illustrates most clearly the specific features of the criterion employed. In our next papers we plan to present the list of galaxy triplets and analyze the properties of groups with four to 400 members, describe our catalog of very isolated LSC galaxies, and specific features of the distribution of voids. Individual TallyFisher distances [6] are already available for about 1700 LSC galaxies [7]. We plan to use these data to analyze non-Hubble motions in the LSC in order to probe the distribution of dark matter on 3-10 Mpc scale lengths.

\section{CRITERIA FOR SELECTING MULTIPLE SYSTEMS OF GALAXIES}

Various algorithms have been suggested to identify groups of galaxies in a magnitudeor distance-limited sample. All these algorithms actually reduce to the following two main ones: percolation (the "friend of friend" method) and taxonometry (construction of a hierarchical tree).

Huchra and Geller [8] used the percolation method by joining galaxies into groups based on the condition that their projected mutual distances and line-of-sight velocity differences should be smaller than certain threshold values $R_{c}$ and $V_{c}$. With $R_{c}=$ $0.52 \mathrm{Mpc}$ and $V_{c}=600 \mathrm{~km} / \mathrm{s}$ the above authors grouped about $74 \%$ CfA galaxies. The resulting groups had a typical size of $R_{H}=$ 1.1 Mpc, line-of-sight velocity dispersion of $\sigma_{v}=208 \mathrm{~km} / \mathrm{s}$, and an average virial mass 
of $\lg \left(M_{v i r} / M_{\odot}\right)=13.5$. Many authors applied this method to different galaxy samples. One of the weak points of the method is freedom in the choice of two percolation parameters, $R_{c}$ and $V_{c}$, whose variation affects substantially the characteristic sizes and masses of the groups and the percentage of galaxies found to be group members. In the percolation algorithm parameters $R_{c}$ and $V_{c}$ trace certain contrast of galaxy number density and overlooks many real groups in low-density regions, while clusterizing small unvirialized aggregates in high-density regions. Another disadvantage of the "friend of friend" methods manifest itself in the form of the strong dependence of group parameters on the distance $D$ to the group. $\mathrm{Nu}-$ merous attempts aimed to reduce this dependence by introducing variable quantities $R_{c}(D)$ and $V_{c}(D)$ resulted in subjectively arbitrary choices. The most recent application of the percolation method to 2MASS [9] galaxies yielded 1258 groups and 1710 pairs of galaxies for a relative density contrast of $\delta \rho / \rho=80$. In general, members of groups and pairs make up for a total of $36 \%$ and $17 \%$ of the entire sample. Groups with $n \geq 5$ elements have a projected radius of about $1 \mathrm{Mpc}$, line-of-sight velocity dispersion on the order of $200 \mathrm{~km} / \mathrm{s}$, and an average virial mass of $\lg \left(M_{v i r} / M_{\odot}\right) \sim 13.5$. At the depth of the 2MASS sample $\left(D_{\max }=140 \mathrm{Mpc}\right)$ the con- tribution of virial masses of groups identified using the percolation method is equal to only $\Omega_{m}=0.10-0.13$. An examination of the list of these groups gives a rise to numerous questions. In particular, we do not understand why Eridanus+Fornax I is the most massive cluster complex instead of Virgo, which we know as the center of the Local Supercluster. Tully [4, 5] and Vennik [10] used another, "taxonometric", method to group galaxies into pairs in accordance with the maximal ratio of luminosity to cubed mutual distance $\left(L_{i k} / R_{i k}^{3}\right)$. The resulting pair was substituted by a "particle" with the luminosity equal to the total of the galaxies and the search for maximal $\left(L_{i k} / R_{i k}^{3}\right)$ was repeated. The process ended by the construction of a single hierarchical "tree" with branches containing the entire galaxy sample considered. Cutting the tree branches at a certain level of the contrast of volume density yielded a set of branches (groups) whose sizes and virial masses depended on the adopted density (luminosity) contrast. Tully [4] used the method of dendrograms to infer a characteristic group radius of $0.32 \mathrm{Mpc}$ and the average line-of-sight velocity dispersion of $M_{v i r} / L_{B}=94 M_{\odot} / L_{\odot}$, which proved to be substantially lower than the average ratio for groups of the Huchra-Geller [8] list. Practical applications of both the percolation and the dendrogram methods ignored individual 
properties of galaxies by viewing them as indistinguishable particles. It is evident that the same threshold values $R_{c}$ and $V_{c}$ would be sufficient (and even redundant) for clustering a pair of dwarf galaxies and, at the same time, evidently insufficient to bind a pair of giant galaxies. Such inadequacy of the criterion distorts the estimates of virial masses.

\section{CLUSTERIZATION ALGORITHM}

Galaxies can be grouped into small systems with their individual properties taken into account by viewing two arbitrary galaxies as a virtual bounded pair [11]. We proceed from this evident premise and require that the difference $V_{i k}$ of the space velocities of galaxies in physical pair and their mutual space distance $R_{i k}$ obey the condition of negative total energy

$$
\frac{V_{i k}^{2} R_{i k}}{2 G M_{i k}}<1,
$$

where $M_{i k}$ is the total mass of the pair and $G$ is the gravitational constant. We correct the squared velocity difference of the pair $V_{i k}^{2}$ for velocity measurement errors. However, observations give us only the line-of-sight projection of velocity $V_{i k}$ and the sky-plane projection of $R_{i k}$. Therefore condition (1) must be supplemented by an additional constraint onto the maximum distance between the components for fixed mass $M_{i k}$. The condition that the components of the pair are located inside the "zero-velocity" sphere [12] has the following form

$$
\frac{\pi H^{2} R_{i k}^{3}}{8 G M_{i k}}<1,
$$

where $H$ is the Hubble constant. Note that both conditions (1) and (2) are conservative with respect to projection factors, i.e., use of projected mutual velocities and distances in formulae (1) and (2) instead of the space velocities and distances does not exclude true (physical) pairs. However, these conditions do not prevent false (optical) pairs from getting into the catalog.

Our algorithm is actually a variant of the percolation method. We first identify all pairs satisfying conditions (1) and (2) and then group all pairs having a common component into a single entity. Finally, if we find a galaxy to be a satellite of several more massive galaxies, we link it to the most massive neighbor. In particular, a group may be a subgroup inside a more massive structure. In this sense, our algorithm combines the advantages of both the "friend of friend" method and hierarchical approach.

We determine the masses of galaxies from their -band IR luminosity assuming that all galaxies have the same "mass-luminosity" ratio:

$$
M / L_{K}=\kappa\left(M_{\odot} / L_{\odot}\right),
$$


where we set $\kappa$ equal to 6 . In our algorithm $\kappa=6$ is actually the only more or less arbitrary quantity. We chose it based on the following assumptions. According to the data of Bell et al. [13], the average cosmic mass-to- $K$-band-luminosity ratio is equal to $0.95 \pm 0.27 M_{\odot} / L_{\odot}$, which agrees well with the results of the computations of Fukijita et al. [14]. According to the data compiled by Karachentsev and Kut'kin [15], for galaxies of the Local Volume the average ratio of the mass inside standard radius $R_{25}$ to the integrated $K$-band luminosity is equal to $\left\langle M_{25} / L_{K}\right\rangle=1.3 \pm 0.2$ in solar units, and this ratio remains almost constant and varies from $1.1 \pm 0.2$ for giant galaxies with the mean luminosity of $1.1 \times 10^{11} L_{\odot}$ to $1.5 \pm 0.2$ for dwarf galaxies with the mean $K$-band luminosity of $1.1 \times 10^{7} L_{\odot}$ (the slight increase of $\left\langle M_{25} / L_{K}\right\rangle$ toward dwarf galaxies is evidently due to higher content of the gaseous component). Flat rotation curves observed for most of galaxies indicate the dominating role of the dark halo beyond the standard radius $R_{25}$. The most extended rotation curves inferred from the data of the 21-cm line emission reach out to $R_{\max }=(3-6) R_{25}[16,17,18]$. These $R_{\max }$ values correspond to the global ratio of $M / L_{K} \simeq 6 M_{\odot} / L_{\odot}$ used in formula (3). Note that we "trained" clusterization algorithm (1-3) by applying it to the de- tailed 3D distribution of galaxies in the Local Volume. Choosing dimensionless parameter $\kappa$ values in the $\kappa<4$ interval drastically reduces the relative number of clusterized galaxies, whereas adopting $\kappa>10$ results in grouping of galaxies into extended and evidently nonvirialized aggregates. With the value $\kappa=6$ adopted in this paper the dwarf companions in known nearby groups are usually located inside the zero-velocity spheres around massive galaxies of the corresponding groups. Note also that the average virial mass-to-luminosity ratio $\left\langle M_{v i r} / L_{K}\right\rangle=17.5 \pm 3.6 M_{\odot} / L_{\odot}$ for eight groups of the Local Volume: Local Group, M81, CenA, M83, IC342, Maffei, LeoI NGC6946 (whose mean luminosity is $\left\langle L_{K}\right\rangle=$ $\left.1.3 \times 10^{11} L_{\odot}\right)$ is almost equal to the typical $M_{v i r} / L_{K}$ ratio that we inferred for small groups of the same luminosity inside the entire volume of the Local Superclusrter. Guzik and Seljak [19] found a similar total mass-to- $K$-band luminosity ratio$\left(17.0 \pm 2.9 M_{\odot} / L_{\odot}\right)$ - for small groups and field galaxies with $\left\langle L_{K}\right\rangle \sim 0.8 \times 10^{11} L_{\odot}$ by analyzing effects of gravitational lensing, and this fact demonstrates the good agreement of the galaxy group mass estimates obtained by two independent methods. 


\section{INITIAL OBSERVATIONAL DATA}

Our main sources of the data on line-ofsight velocities, apparent magnitudes, morphological types, and other parameters of galaxies are the HyperLEDA ${ }^{1}$ [20] and NED ${ }^{2}$ databases. Note that these databases contain a substantial number of objects with erroneous line-of-sight velocities adopted from automatic sky surveys like 6dF. Cases of confusion of coordinates and velocities are rather common for galaxies located closely to each other on the celestial sphere. The apparent magnitudes and line-of-sight velocities in the SDSS survey often correspond to individual knots and associations in bright galaxies. These effects are very important for selecting of true close pairs of galaxies. We took these effects into account and made necessary corrections, where possible. This proved to be most time-consuming part of our work. Because the databases are permanently updated with new (and sometimes erroneous) data, it is necessary to repeat the correction of information. That is why we fixed the sample of initial data as it was in mid-2006 (i.e., June, 2006).

We independently found optical identifications for many $H I$ sources of the HIPASS

\footnotetext{
1 http://leda.univ-lyon1.fr

2 http://nedwww.ipac.caltech.edu/
}

survey by refining their coordinates and determining the apparent magnitudes and morphological types of galaxies [21]. We examined many dwarf galaxies, especially those with low surface brightness, on the DSS digital images in order to find their principal parameters. The typical error of our visual estimates of galaxy magnitudes is about $0.5^{m}$, and the average error of the inferred type is about \pm 2 in the numeric scale employed by de Vaucoulers in the $\mathrm{RC} 2$ catalog [22]. The best indicator of the baryonic mass of a galaxy is known to be its infrared magnitude, which depends only slightly on the amount of dust and the number of young stellar complexes. Thus we adopted the longest-wavelength, $K$-band $(\lambda=2.16 \mu \mathrm{m})$ part of the all-sky 2MASS [23, 24] as our main source of photometry. We converted the estimates of galaxy magnitudes in other optical $(B, V, R, I)$ and near-infrared bands $(J, H)$ into the $K$-band magnitude using syntehtic galaxy colors of Bizzoni [25] and Fukujita et al. [26]. The greatest amount of photometric data is available in the $B$ band. We use the following relations between the $B-K$ color excess and morphological type discussed by Jarett et al. [24] and Karachentsev and Kut'kin [15] :

$\langle B-K\rangle=+4.10$, for galaxies of types $T \leq$ 2 (i.e., E, S0, and Sa), which are dominated by bulges, 
$\langle B-K\rangle=+2.35$ for late-type galaxies $T \geq 9$ (i.e., Sm, Im, Irr), and

$\langle B-K\rangle=4.60-0.25 \times T$ for intermediatetype $(T=3-8)$ objects.

Note that due to the short exposures the 2MASS survey proved to be insensitive to low-surface brightness and blue galaxies. For about one thousand dwarf and spheroidal galaxies recently discovered by Karachentseva et al. [27, 28] in the volume of the Local Supercluster, only eye-estimated $B$ band magnitudes are available, which we converted into the $K$-band magnitudes using the method described above. Despite the lack of good photometry for these objects, gas-rich dIr galaxies have accurate 21-cm line radial velocities and they are important "test particles". Due to the low luminosities of dwarf galaxies, large errors of estimated magnitudes have virtually no effect on the results of clusterization performed using our algorithm.

We collected all the line-of-sight velocity measurements available in the HyperLEDA and NED databases for galaxies in the Local Supercluster and its neighborhood. We excluded unreliable and inaccurate measurements, which velocity-measurement error exceeds $75 \mathrm{~km} / \mathrm{s}$. In automatic surveys (SDSS, $2 \mathrm{dF}$, and $6 \mathrm{dF}$ ) we also excluded the measurements with velocities smaller than $600 \mathrm{~km} / \mathrm{s}$, because they mostly represent Milky-Way stars projected onto distant galaxies. If sev- eral line-of-sight velocity measurements were available for a galaxy, we chose the median one, the velocity error was estimated as the dispersion of all measurements with the exclusion of the outliers.

Our initial sample cleaned from unreliable and doubtful cases contained a total of 10403 galaxies with the line-of-sight velocities $V_{L G}<3500 \mathrm{~km} / \mathrm{s}$, located at the Galactic latitudes $|b|>15^{\circ}$. For all these galaxies the apparent magnitudes and morphological types have been found. To prevent the distortion of the clusterization process at the boundary of the volume considered, we also use the data on the galaxies located in the boundary regions with $10^{\circ}<|b|<15^{\circ}$ and with $3500<V_{L G}<4000 \mathrm{~km} / \mathrm{s}$, because they may contain some of the members of galaxy groups with high virial velocities. Grouping criteria (1-3) allowed us to identify within the Local Supercluster volume a total of 1018 galaxies belonging to binary systems.

\section{THE CATALOG OF 509 NEARBY GALAXY PAIRS}

The Table contains the binary galaxies that we identified using our criteria. The first column gives the number of the pair in the catalog. The second column gives the name of the galaxy or its number in the wellknown NGC, IC, UGC, CGCG, ESO, PGC, 
and DDO catalogs or in the 2MASS, 6dF, APMUKS, SDSS, IRAS, and other sky surveys as given in the NED. Note that for practical reasons we omit the coordinate part of the galaxy name used in surveys. The third column gives the galaxy coordinates for the epoch of 2000.0. The fainter component of the pair follows the primary component and the pairs are sorted in right ascension. Columns (4) and (5) give the line-of-sight velocity of the galaxy (in $\mathrm{km} / \mathrm{s}$ ) with respect to the centroid of the Local group and its standard error, respectively. Columns (6) and (7) give the coded morphological type and the apparent $K$-band magnitude corrected for Galactic extinction according to Schlegel et al. [29], respectively. Column (8) gives the projected separation between the galaxies in kpc. Column (9) gives the logarithm of the total $K$-band luminosity of the pair. Columns (10) and (11) give the orbital mass-to-luminosity ratio with and without velocity measurement correction, respectively. We compute the mass by the following formula [30]

$$
M_{p}=\frac{16}{G \pi} \Delta V^{2} R_{\perp}
$$

using the component line-of-sight velocity difference and projected linear separation. We compute the distance to the pair from the average line-of-sight velocity with respect to the centroiod of the Local group with the adopted Hubble-constant value of $H=73 \mathrm{~km} / \mathrm{s} / \mathrm{Mpc}$. Column (12) gives the logarithm of the smallest of the values given by criteria (1) and (2) for the given pair with respect to the surrounding galaxies. The higher is this quantity, the greater is the degree of isolation of the considered pair. A close-to-zero value implies that the pair is at the threshold of the formation of a bigger structure ("capturing" of a new member or "joining" other groups). We compute the total luminosity of the pair assuming that the absolute magnitude of the Sun is $K_{s}=3.28^{m}$ [31]. We omit the negative unbiased estimated of the orbital mass for the pairs with line-of-sight velocity differences smaller than the corresponding measurement errors.

\section{DISTRIBUTIONS OF THE PRINCIPAL PARAMETERS OF THE PAIRS}

The fraction of galaxies that are members of binary systems in the Local Supercluster and its neighborhood is about $10 \%$, which is somewhat lower than the corresponding values $12-17 \%$, according to the data of Huchra and Geller [8], Crook et al. [9], Magtesyan [32], and Gourgoulhon et al. [33]. Figure 1 shows the distribution of the mean line-of-sight velocity of the pairs. The median 
of this distribution is equal to $2389 \mathrm{~km} / \mathrm{s}$. Figure 2 shows the map of the distribution of pair centers on the sky in equatorial coordinates. The region of strong Galactic absorption is shown by gray color. It is obvious from this map and from 3D distribution (Fig. 3) that the pairs do not reveal clear large scale structure. However, on short scale lengths in the vicinity of $1 \mathrm{Mpc}$, the pairs exhibit excess of mutual association compared to uniform random distribution. The pairs $21+22$ and $194+195$ are examples of such close associations.

Figure 4 shows the distribution of the line-of-sight velocity difference between the satellite and the primary component of the pair. The distribution has quite symmetric shape with a mean difference of $-1 \pm 3 \mathrm{~km} / \mathrm{s}$, which indicates that our sample exhibits no excess of positive velocities discussed by Arp [34] in cases of the companions of M31 and other galaxies. The root-mean-squared velocity difference for the components of 509 pairs is equal to $\sigma_{v}=62 \mathrm{~km} / \mathrm{s}$. Note that in the vicinity of zero the distribution is much sharper than a Gaussian. Note also that for about $60 \%$ of all pairs the velocity difference is smaller than its error of measurement. For such pairs unbiased estimates of orbital masses are negative and we do not give them in Column 11 of the table. Taking into account broadening of distribution by errors, the true distribution in Fig. 4 must have an even sharper peak at zero velocity difference. Note the measurement errors of velocity must be reduced several times to improve the estimation of mean mass of pair.

Figure 5 shows the distribution of the projected linear separation between the components in 509 pairs. The median and mean separations are equal to 123 and $177 \mathrm{Mpc}$, respectively, and the separation in the widest pairs reaches $1 \mathrm{Mpc}$. In general, the population of binary galaxies outlines fairly well the linear size of a typical dark halo in the $\Lambda \mathrm{CDM}$ model. The distribution $N\left(R_{\perp}\right)$ can be fitted by a power law with exponent an $\alpha=-1.1$. Figure 6 shows the two-dimensional distribution of the line-of-sight velocity difference and projected separation between the components of the pairs in logarithmic scale. Despite the strong effect of projection factors, pairs show a tendency toward a decrease of $\Delta V$ with decreasing separation between the components, however, the slope of the regression line (the dashed line) is very close to zero and the line differs significantly from the Keplerian law (the solid line). This envelope corresponds to values $\Delta V=121 \mathrm{~km} / \mathrm{s}$ and $R=750 \mathrm{kpc}$, which is close to Andromeda and Milky Way as wide pair of galaxies. Note that normalization of $\Delta V$ by the total luminosity of the pair makes the slope of the regression line closer to the Keplerian value. 


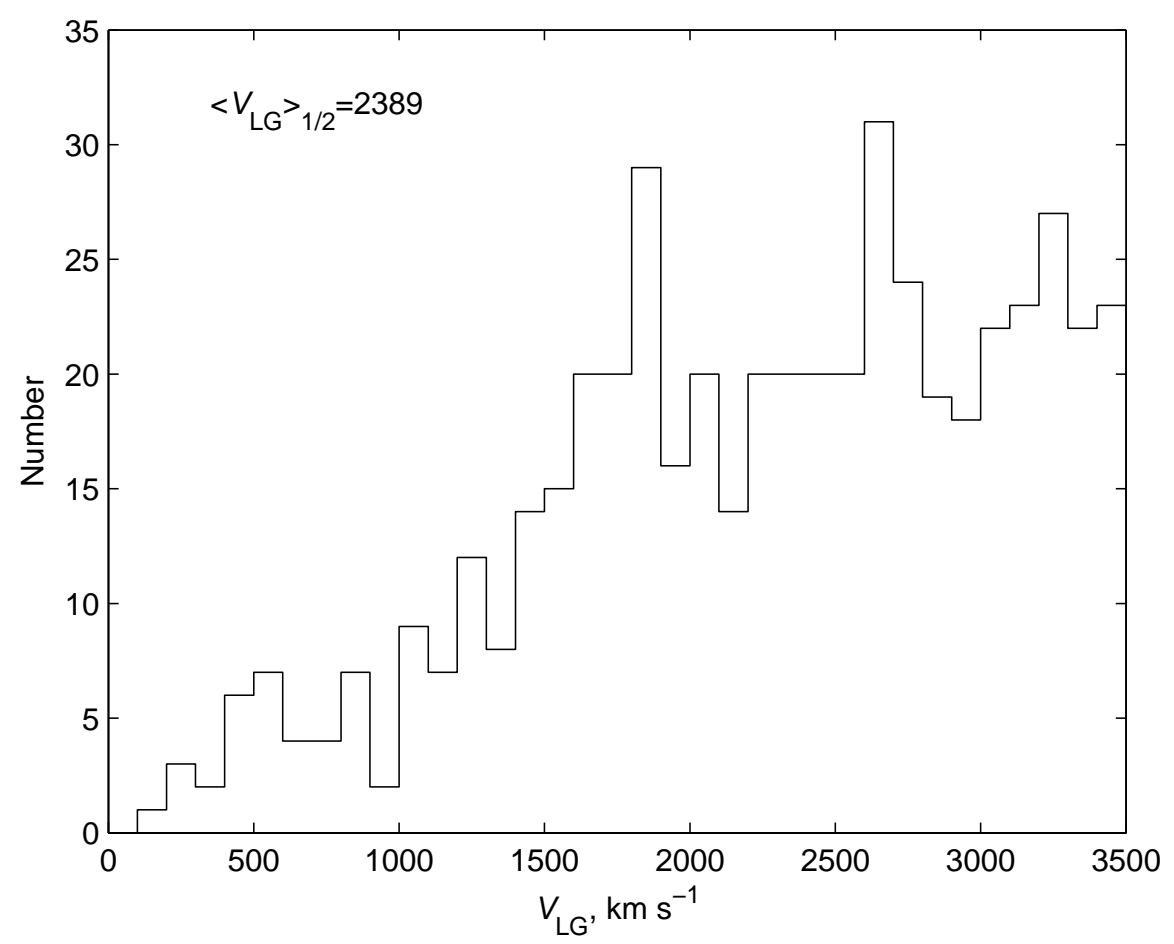

Figure 1: The distribution of the average line-of-sight velocities of pairs with respect to the Local Group.

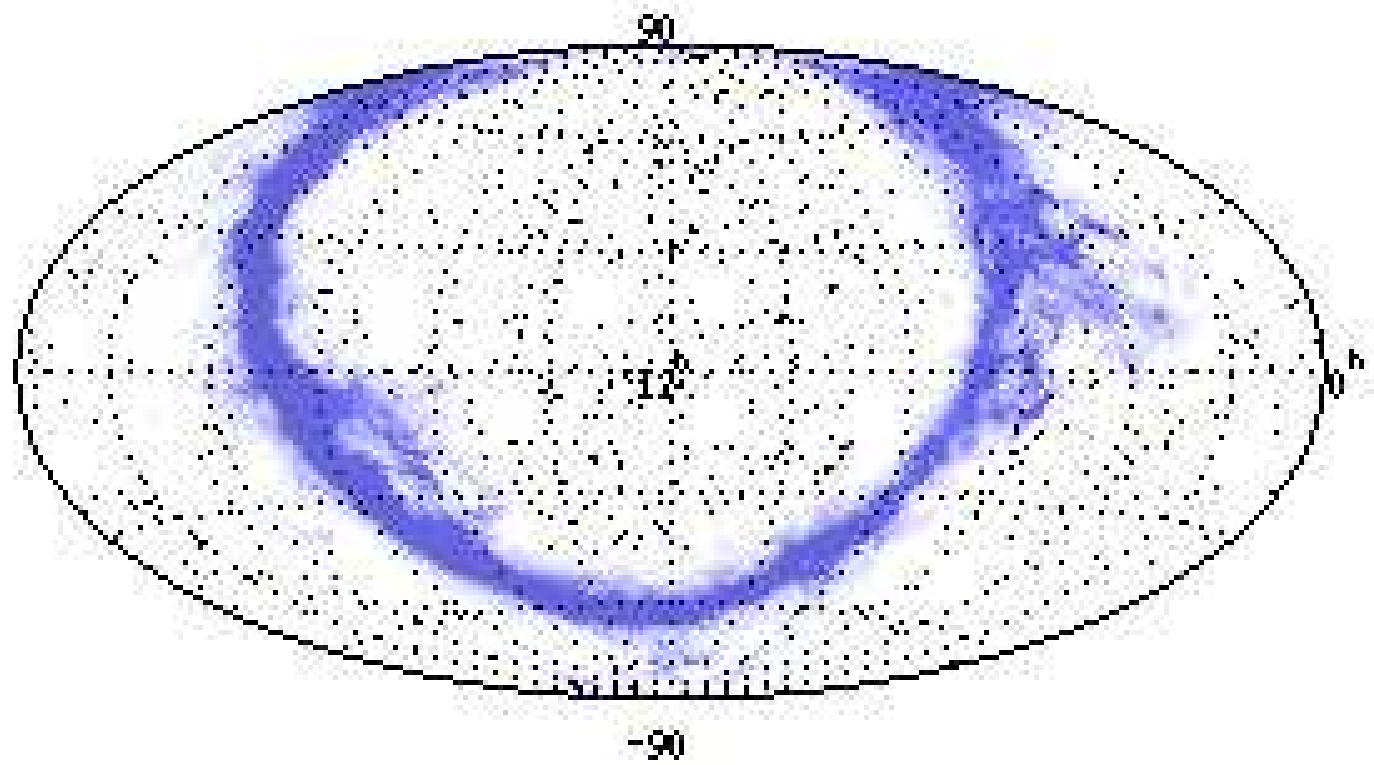

Figure 2: The celestial distribution of pairs in the Local Supercluster in equatorial coordinates. The clumpy gray region indicates the domain of strong Galactic absorption. 

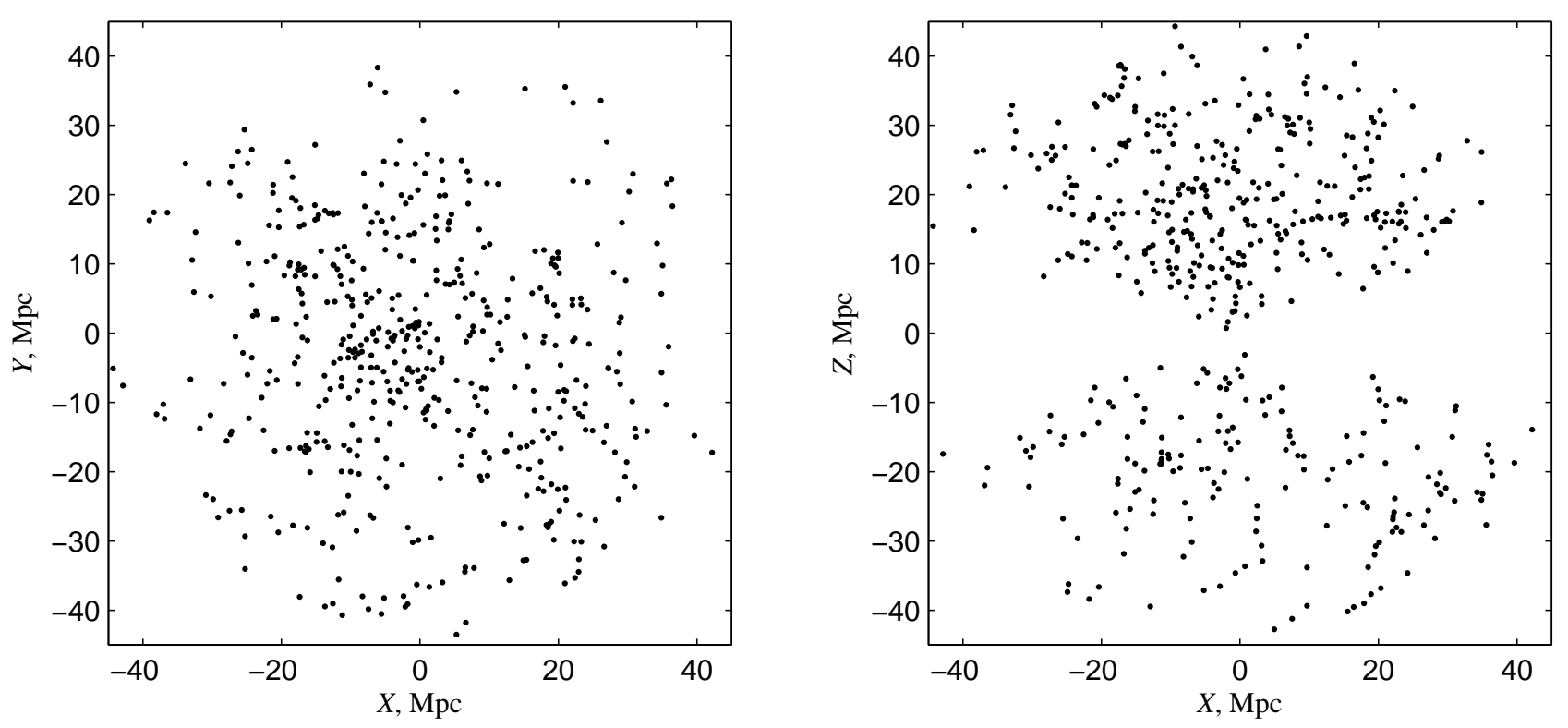

Figure 3: Projected distribution of pairs in the Local Supercluster in Cartesian coordinates.
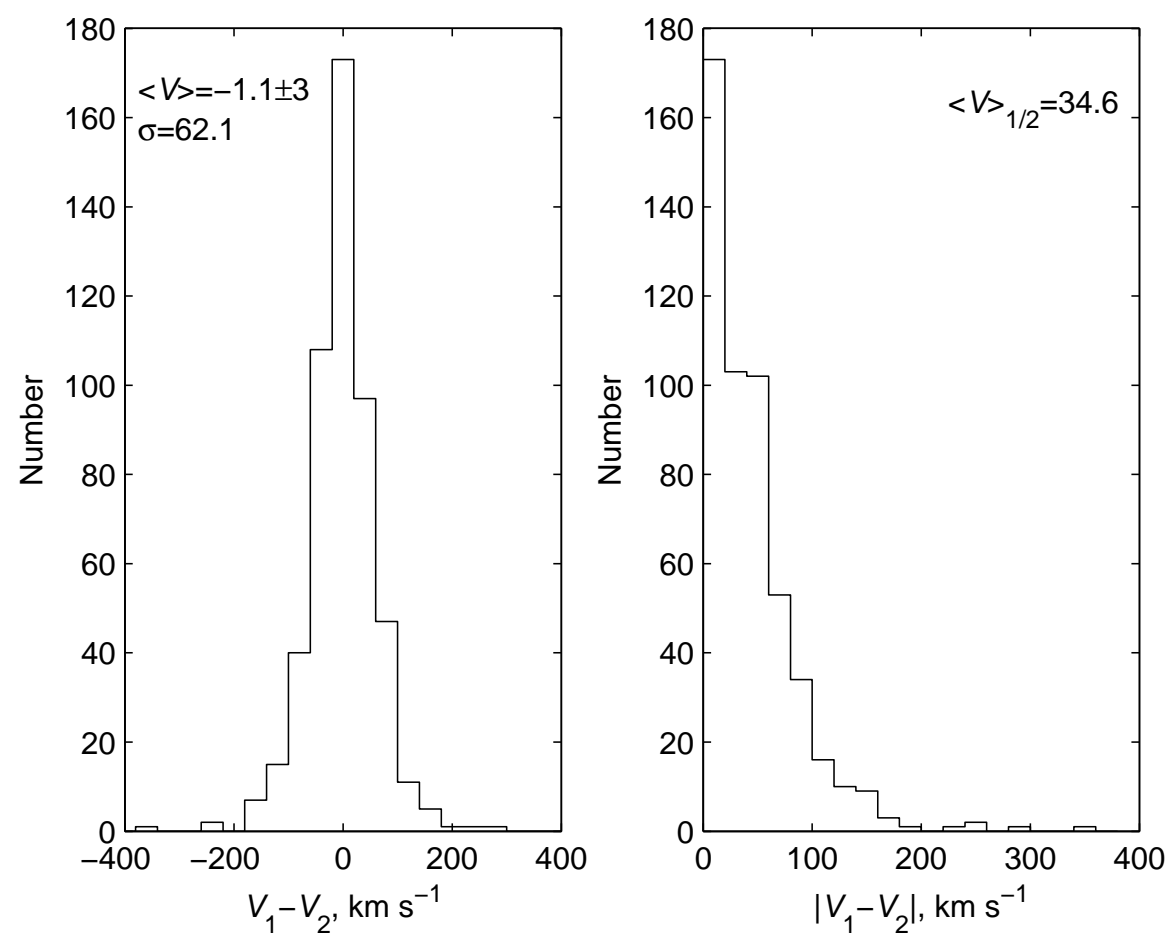

Figure 4: The distribution of the companion minus primary line-of-sight velocity difference.

The left- and right-hand panels in Figure 7 pairs. Both distributions fill more or less unishow the distributions of the visual (left) and formly a wide sector of possible values over absolute (right) $K$-band magnitudes of the 10-magnitude range. About $40 \%$ of all pairs bright (1) and faint (2) components of the components differ in luminosity less than a 


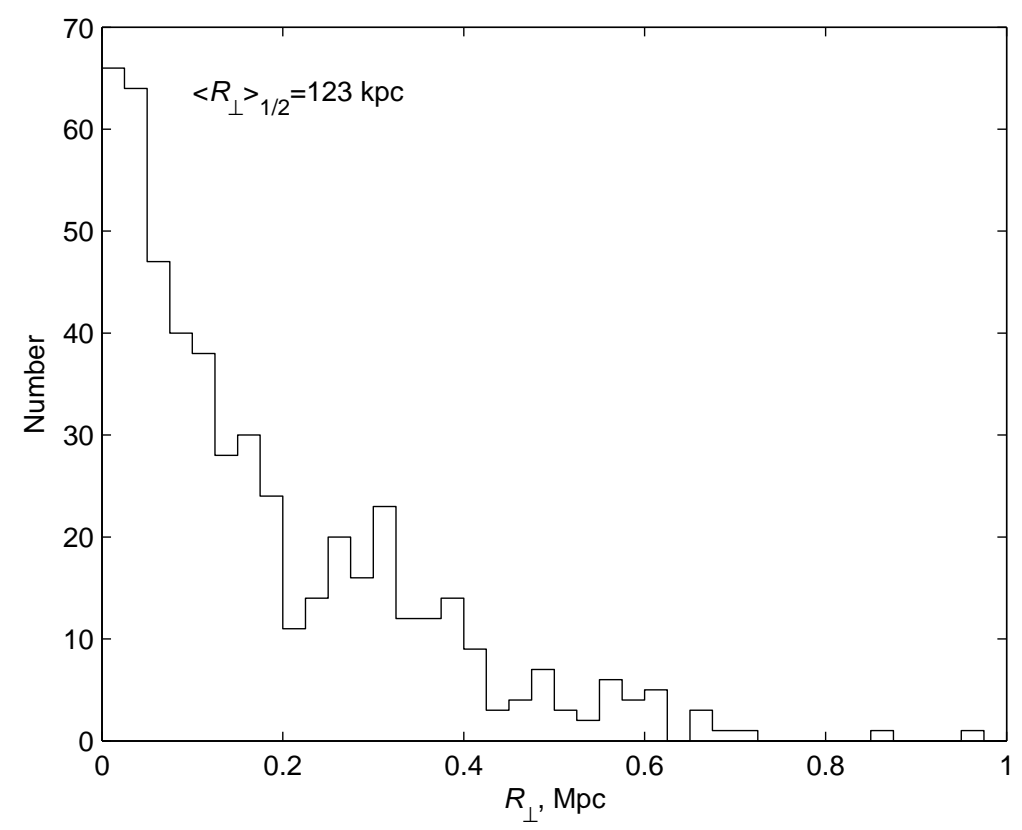

Figure 5: The distribution of the projected separation between the components in the pairs of the Local Supercluster.
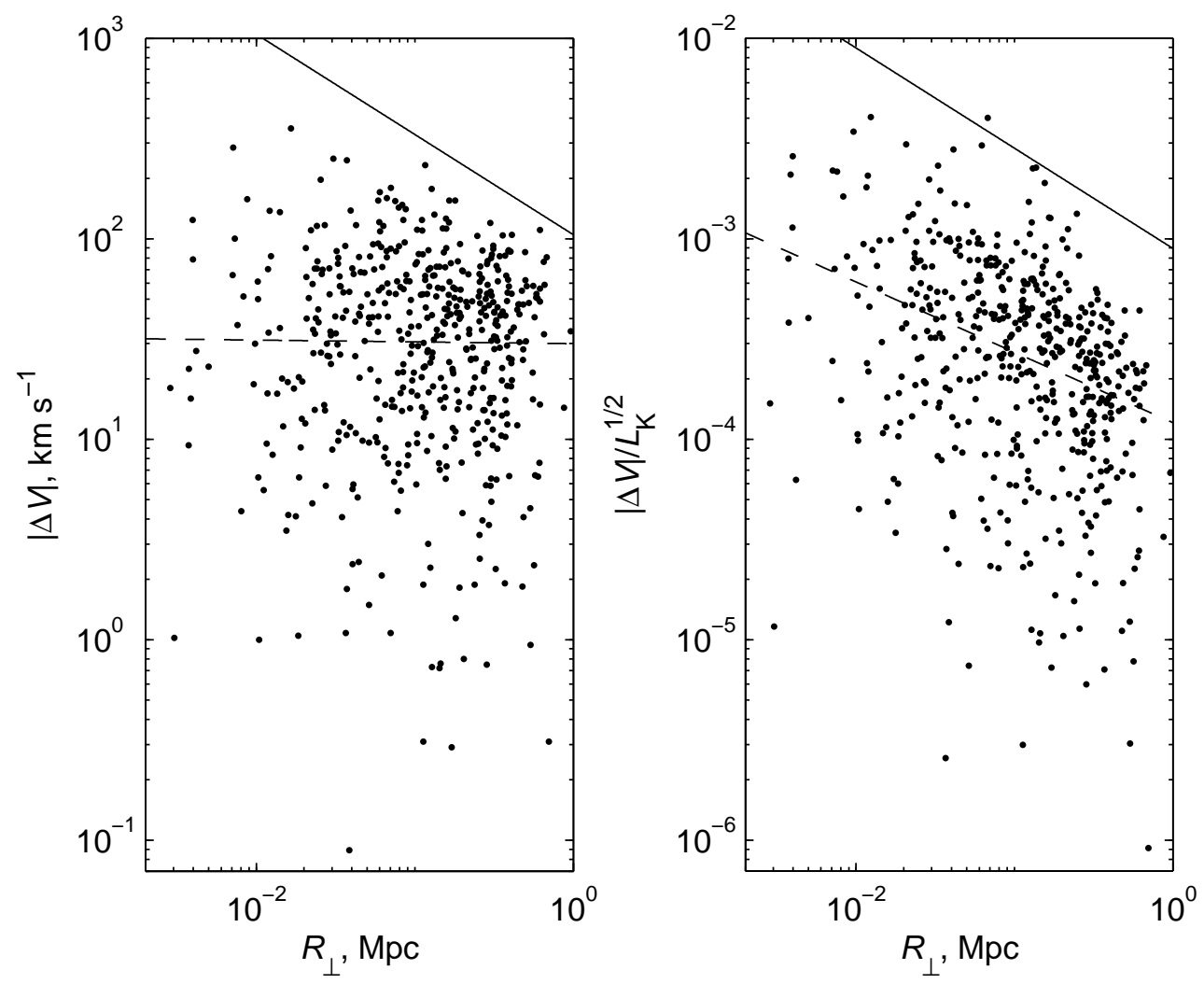

Figure 6: The distribution of the line-of-sight velocity difference and projected separation for the pairs of the Local Supercluster. 

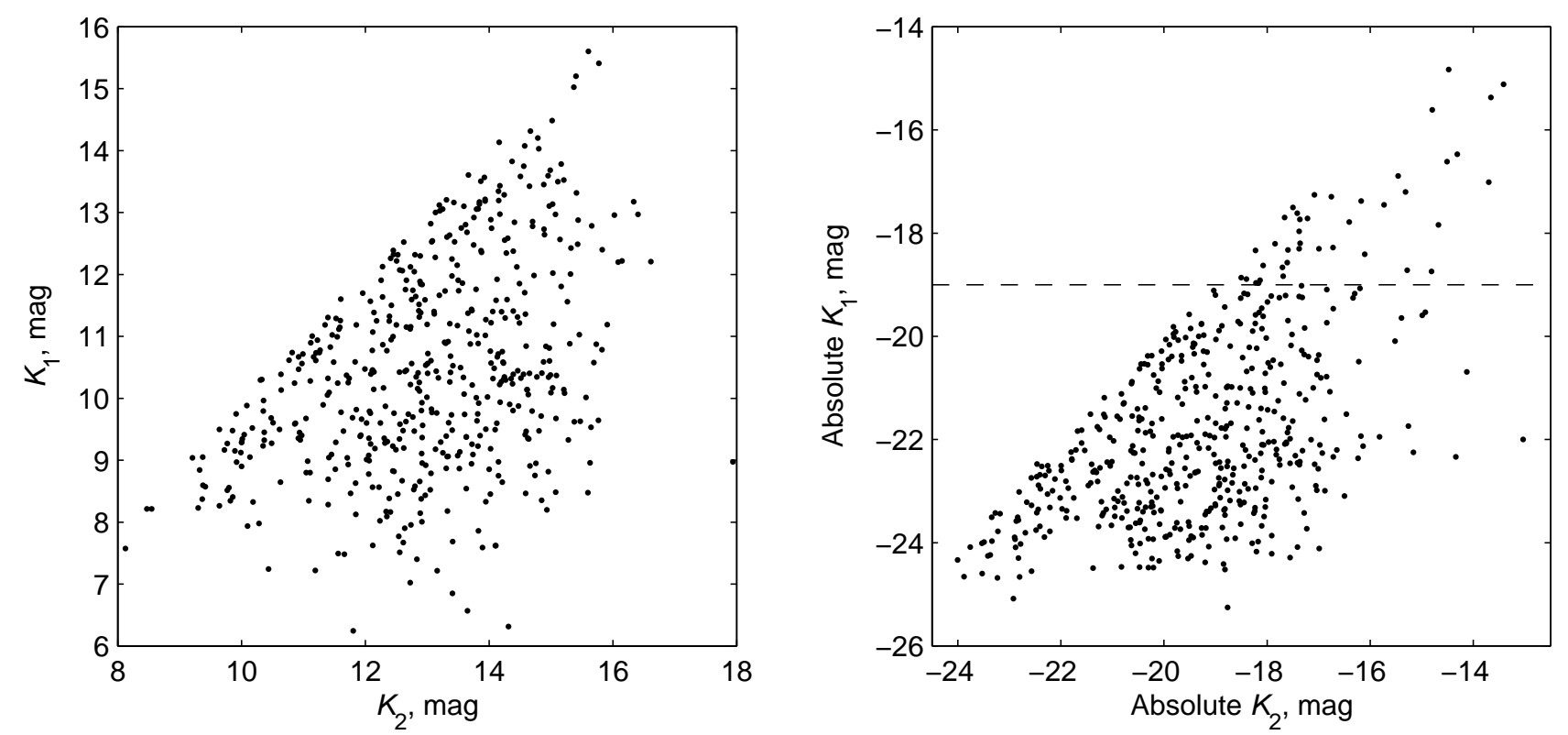

Figure 7: Distribution of the apparent (left) and absolute (right) $K$-band magnitudes of the bright (1) and faint (2) components in the Local Supercluster.

10 times. At the same time, there are galaxies, e.g., NGC 3044, NGC 2683, and NGC 3621, with dwarf companions that are 7-10 magnitudes fainter than the primary component. The special interest are drawn by the pairs located in the top right corner of the right-hand panel in Fig. 7. The dashed line in this panel corresponds to $M_{K}=-19.0$ (the luminosity of the SMC). Pairs that are located above this line have both components that are dwarf galaxies. There are total of 41 pairs like UGC 5272 and its companion (pair No. 159), which are located in the domain of lower-than-SMC luminosity. The pair of metal-poor BCD dwarfs SAO0822+3545 and SDSSJ0825+3532 (pair No. 113) studied by Cnehgalur et al. [35] is another example. Al- most all these binary dwarfs are gas rich and contain young blue stellar population. The average component line-of-sight velocity difference for these pairs is equal to only $25 \mathrm{~km} / \mathrm{s}$, and the median and mean projected separations are equal to 30 and $42 \mathrm{kpc}$, respectively. These pairs of blue dwarfs with considerable reserves of gas and active star formation may be a sort of small multiple systems at a stage close to component merger. On the other hand, Tully et al. [36] pointed out the presence in the Local Volume of groups which exclusively consist of dwarf galaxies. Note that old percolation criteria proved to be insensitive to multiple dwarf systems. They were discovered as a result of, among other things, in-depth analysis of the 
population of the Local Volume and of use a more refined algorithm for group searching. It is evident that $H I$-observations with high angular resolution would be a promising method for analyzing the kinematics and evolutionary status of dwarf pairs pairs and groups.

Figure 8 shows the distribution of the morphological types of the bright (1) and faint (2) components of 509 pairs. On the average, the primary galaxy have an earlier type $\left(\left\langle T_{1}\right\rangle=3.8\right)$ than its companion $\left(\left\langle T_{2}\right\rangle=6.9\right)$. This fact is easy to explain by the well-known correlation between the luminosity and morphological type of galaxies. The lines of direct and reverse regression in Fig. 8 point out a weak correlation between the morphological types of the components, which also can be due to the luminosity effect.

Figure 9 shows the distribution of estimated orbital masses and orbital mass-toluminosity ratios for galaxy pairs inferred in accordance with formula (4). The median mass of the pairs is $1.5 \times 10^{11} \mathrm{M}_{\odot}$, and the median mass-to-luminosity ratio is $11.3 M_{\odot} / L_{\odot}$, which is almost twice the $\kappa=6$ value that we adopt for individual galaxies. However, these mass estimates are statistically biased. We already pointed out above that the line-of-sight velocity difference is smaller than its standard error for more than half of all pairs. To obtain an unbiased mass estimate, we must substitute $\left(V_{12}^{2}-\sigma_{1}^{2}-\sigma_{2}^{2}\right)$ for $V_{12}^{2}$ in formula (4). In this case mass estimations become negative for $60 \%$ of the pairs and the median mass-to-luminosity ratio also becomes negative $\left(-3 M_{\odot} / L_{\odot}\right)$. The distributions of $M$ and $M / L$ for the pairs corrected to for velocity measurement errors are shown in gray, but they represent only the domain of positive masses. All these data illustrate the fact that the accuracy of lineof-sight velocities measured in modern optical spectroscopic galaxy surveys (2dF, SDSS, $6 \mathrm{dF}$ ), which is about $50 \mathrm{~km} / \mathrm{s}$, is clearly insufficient to compute a bona fide average mass of galaxy pairs. Obviously the special observations of binary galaxies are needed to reduce the velocity errors down to $5-10 \mathrm{~km} / \mathrm{s}$.

Figure 10 shows how the line-of-sight velocity difference, projected separation between the components, and the orbital massto-luminosity ratio of binary galaxies vary with heliocentric distance. It is evident that in our sample the fraction of pairs consisting of two dwarf components decreases appreciably toward the outskirts of the volume considered. However, the average dynamical parameters of the ensemble of galaxy pairs vary little with distance. This fact again demonstrates the advantage of our criterion, which takes individual properties of galaxies into account. 


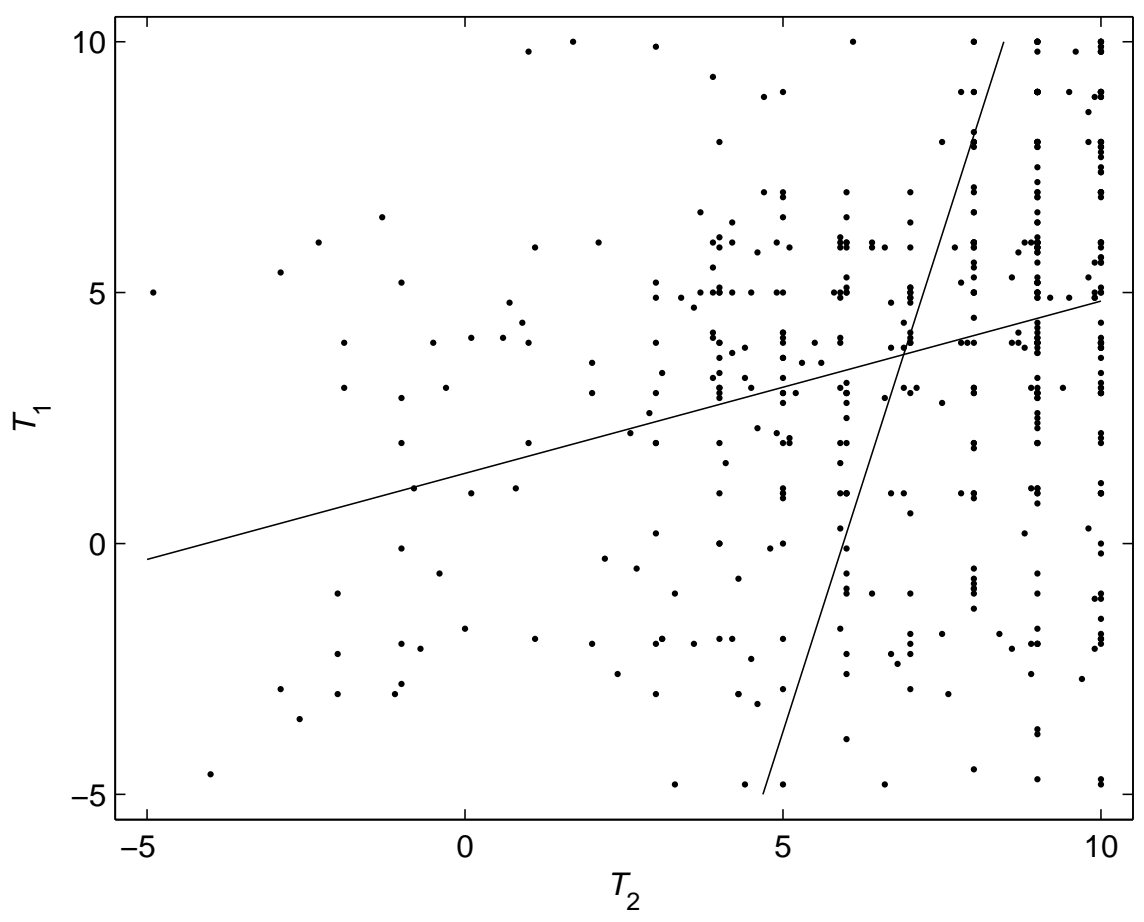

Figure 8: Distribution of the types of the bright (1) and faint components. The lines show the direct and invrse regression
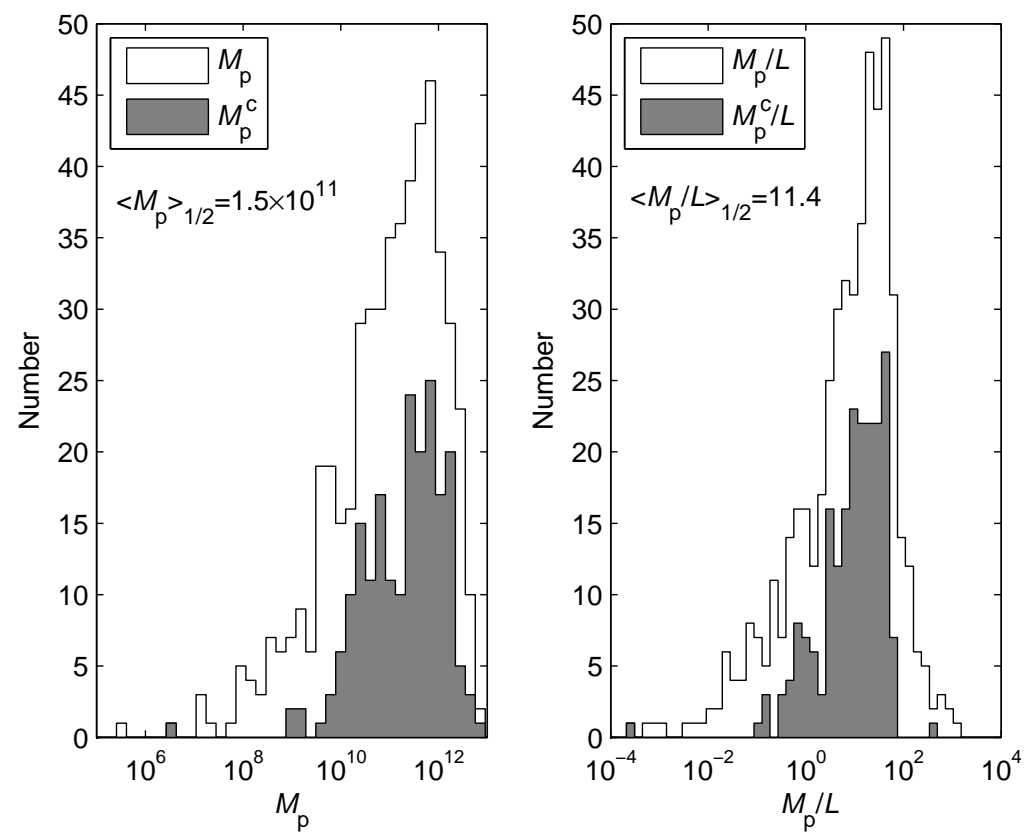

Figure 9: Distribution of estimated orbital masses and orbital mass-to-luminosity ratios for the pairs of the Local Supercluster. 


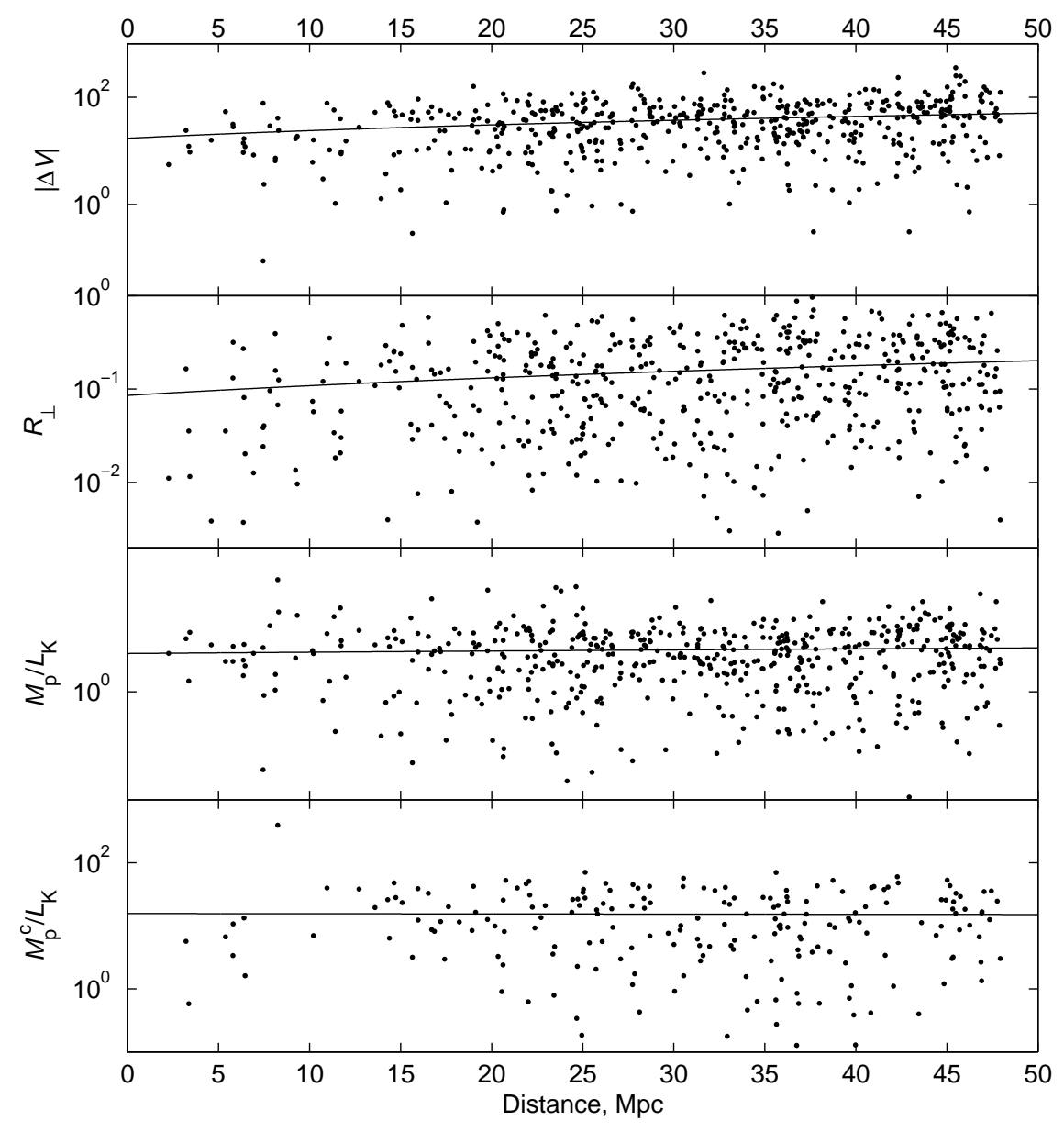

Figure 10: Characteristic diagrams showing how the properties of identified galaxy pairs vary with distance.

VII. COMPARISON WITH OTHER SAMPLES OF GALAXY PAIRS

The most detailed study of binary galaxies was performed by Karachentsev [37]. He compiled a catalog of 603 isolated Northernsky pairs with components brighter than $B=$ $15.7^{m}$ (they are denoted as KPG in the NED database). These pairs were identified on the condition that they are isolated with respect to nearby projected galaxies without invoking line-of-sight velocity data. Later Reduzzi and Rampazzo [38] used the same criterion to identify a total of 409 pairs in the Southern sky (these pairs are denoted as RR in the NED database). The isolation condition favored the identification of closer binary systems with brighter components. The characteristic depth of the KPG sample is 6350 $\mathrm{km} / \mathrm{s}$, which is substantially greater than the Local Supercluster sample considered in current paper. After taking into account various selection effects in the catalog of isolated 


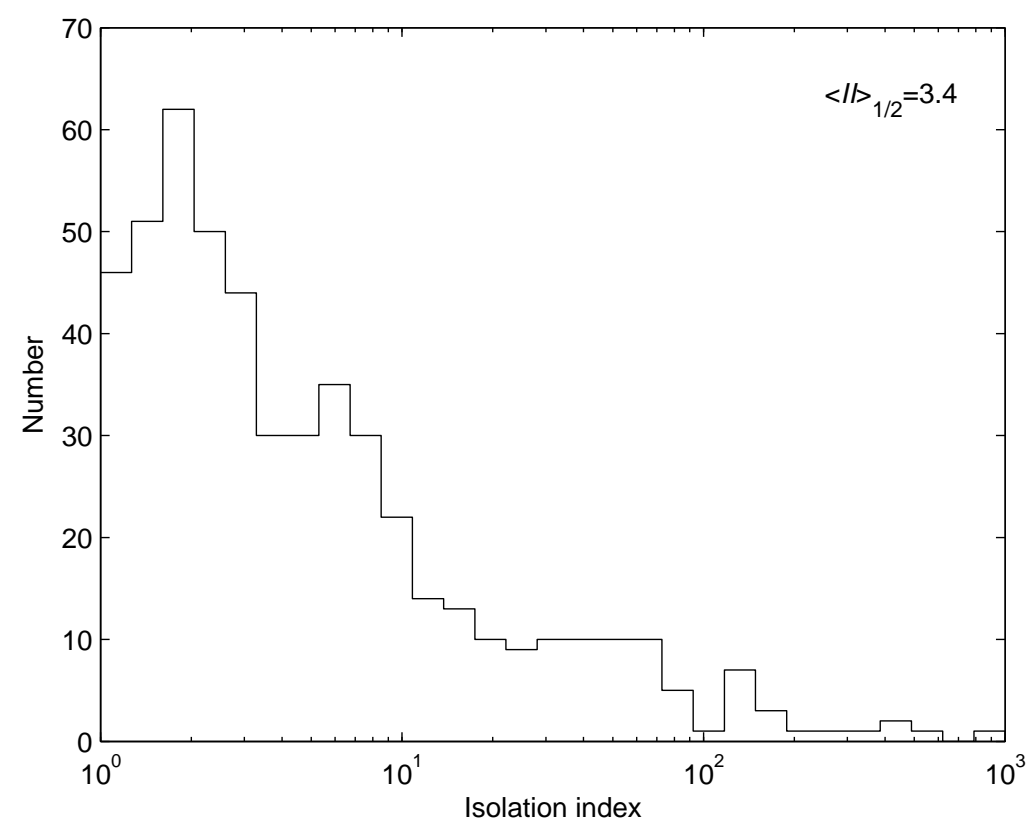

Figure 11: Distribution of the index of isolation of the pairs of the Local Supercluster with respect to neighboring galaxies.

pairs we estimate the fraction of galaxies in tive closeness of the pair members. However, binary systems to be $12 \pm 2 \%$. The KPG pairs the distances of the NGC 4449 and UGC 7577 with their order-of-magnitude higher lumi- galaxies (pair No. 281) (4.21 and 2.54 Mpc, nosities (about $1.3 \times 10^{11} L_{\odot}$ if transformed respectively) indicate that these galaxies are into the $K$-band values) have large line-of- just accidentally located along the same line sight velocity differences, $\left\langle V_{12}\right\rangle=120 \mathrm{~km} / \mathrm{s}$. of sight. Despite the small line-of-sight veWith the average component separation of locity difference $(252 \mathrm{~km} / \mathrm{s}$ and $240 \mathrm{~km} / \mathrm{s})$ only $\left\langle R_{\perp}\right\rangle=40 \mathrm{kpc}$, isolated pairs have a and rather isolated location $(I I=2.3)$ these moderate orbital mass-to-luminosity ratio of members of the CVnI cloud cannot be viewed $\left\langle M / L_{B}\right\rangle=7.8 M_{\odot} / L_{\odot}$ and almost do not as an isolated physical pair.

show evidence for dark halo on these scale lengths.

Among the isolated pairs of the KPG catalog there are several known nearby pairs, in

Our list contains a total of 16 pairs located within the rather thoroughly studied Local Volume. In cases where individual dis- terion (1-3) clusterizes these galaxies, it nevtances have been measured for components of ertheless changes their status from pairs to nearby pairs these distances confirm the rela- groups, due to the presence of other dwarf 
companions. Note that the properties of multiple galaxies identified using a certain criterion in the Local Volume differ from the corresponding properties of multiple galaxies found in deep samples due to the decreasing detection rate of dwarf objects with distance. That may be why the fraction of galaxies in binary systems, $12-17 \%$, in about $100 \mathrm{Mpc}$ deep samples is somewhat higher than in the Local Supercluster $(10 \%)$ or Local Volume $(7 \%)$.

\section{CONCLUSIONS}

We identified the galaxy pairs that we included in our catalog without using the condition of isolation. Therefore, as new dwarf galaxies are found in the volume of the Local Supercluster and new line-of-sight velocity measurements are made for galaxies located in the vicinity of the pars, the list of pairs will be updated by including new objects and some pairs will be promoted to a higher multiplicity category. However, our sample still gives a correct idea about the kinematics of the smallest, most simple systems within the $95-\mathrm{Mpc}$ diameter volume. About $40 \%$ of the considered pairs have dimensionless parameters $I I>5$, which allow us to treat these objects as sufficiently isolated systems (Fig. 11). With the median mass of the pair, $1.5 \times 10^{11} M_{\odot}$, and median projected compo- nent separation of $R_{\perp}=123 \mathrm{kpc}$, the typical density contrast in the ensemble of our pairs is $\delta \rho / \rho_{c} \sim 500$ in the units of critical density. At such contrast systems of galaxies can be considered to be dynamically detached from Hubble flow. On the other hand, with the median absolute value of the component line-of-sight velocity difference of 35 $\mathrm{km} / \mathrm{s}$ and with the median projected separation of $123 \mathrm{kpc}$, a typical pair of our catalog is characterized by the "crossing time" of $3.5 \times 10^{9} \mathrm{yr}$. Hence the components of a typical pair could make about of four turns about the common mass center.

The use of the new clusterizetion algorithm, which takes into account individual properties of galaxies, allowed us to discover a surprisingly large number of pairs consisting of dwarf galaxies. Many components of these systems, which are located far from normal galaxies, are rich in gas and are characterized by active star formation. Such binary dwarf galaxies (for instance, IZw18) have been known long ago. Among of them the galaxies with very low metallicity [39] occur quite often. Our list of galaxy triplets with velocities $V_{L G}<3500 \mathrm{~km} / \mathrm{s}$ also contains triple systems of dwarfs with blue components. Many properties of our systems of dwarf galaxies do not differ from the corresponding properties of associations of nearby dwarfs as described by Tully et al. [36]. The 
closest example of the objects of this population is located at the boundary of the Local group and includes NGC 3109, Sex A, Sex B, and Antlia. The mutual velocities of these "dark groups" are equal to only 10 km/s, i.e., they are comparable to velocity measurement errors. The evolutionary status of multiple dwarf galaxies still remains totally unclear. According to the results of numerical simulations performed by Bekki [40], the evolution of such dwarf systems with extended gaseous envelopes may be governed by their consequent mergers triggering the star-formation bursts. We consider mass 21-cm line observations of these objects on aperture synthesis radio telescopes with a resolution of about $1 \mathrm{~km} / \mathrm{s}$ is very perspective. 
APPENDIX

Table I: Table. Catalog of binary galaxies in the Local Supercluster and its neighborhood.

\begin{tabular}{|c|c|c|c|c|c|c|c|c|c|c|c|}
\hline No. & Name & J2000.0 & \begin{tabular}{|c|}
$V_{L G}$ \\
$\mathrm{~km} \mathrm{~s}$
\end{tabular} & & $\mathrm{~T}$ & $\begin{array}{r}K \\
\mathrm{mag}\end{array}$ & $\begin{array}{l}R_{\perp} \\
\mathrm{kpc}\end{array}$ & $\begin{array}{r}\log L \\
L_{\odot}\end{array}$ & $\begin{array}{r}\frac{M}{L_{K}} \\
\odot\end{array}$ & $\begin{array}{r}\frac{M^{c}}{L_{K}} \\
\odot\end{array}$ & $\log (I I)$ \\
\hline 1 & NGC7820 & J000430.8+051201 & 3252 & 39 & 1 & 9.60 & 507 & 10.81 & 30.9 & 25.6 & 0.30 \\
\hline & UGC00027 & J000428.8+055050 & 3310 & 5 & 6 & 12.25 & & & & & \\
\hline 2 & $6 \mathrm{dF}$. & J000432.0-220503 & 3154 & 74 & 10 & 13.05 & 217 & 9.55 & 320.8 & & 1.41 \\
\hline & ESO538-021 & J000545.9-220442 & 3220 & 10 & 6 & 13.80 & & & & & \\
\hline 3 & UGC00132 & J001400.9+125746 & 1896 & 9 & 8 & 12.85 & 72 & 9.08 & 15.1 & 5.7 & 0.78 \\
\hline & PGC138138 & J001354.2+124827 & 1910 & 5 & 10 & 14.70 & & & & & \\
\hline 4 & ESO078-022 & J002056.8-635126 & 1632 & 20 & 4 & 11.18 & 226 & 9.64 & 37.5 & & 0.88 \\
\hline & $6 \mathrm{dF} \ldots$ & J001636.1-641138 & 1608 & 74 & 9 & 12.50 & & & & & \\
\hline 5 & UGC00260 & J002702.9+113502 & 2337 & 5 & 6 & 10.33 & 23 & 10.21 & 5.8 & 3.4 & 1.67 \\
\hline & CGCG... & J002653.2+113424 & 2277 & 20 & 5 & 13.18 & & & & & \\
\hline 6 & ESO079-003 & J003202.2-641512 & 2474 & 13 & 3 & 9.01 & 83 & 10.82 & 32.4 & 28.3 & 1.85 \\
\hline & ESO079-002 & J003201.1-642325 & 2622 & 27 & 7 & 12.02 & & & & & \\
\hline 7 & UGC00320 & J003230.9+023427 & 2541 & 5 & 6 & 12.88 & 144 & 9.29 & 4.4 & & 2.14 \\
\hline & APMUKS... & J003145.4+024253 & 2548 & 20 & 9 & 15.44 & & & & & \\
\hline 8 & NGC0148 & J003415.5-314710 & 1842 & 17 & -2 & 9.05 & 232 & 10.63 & 5.4 & & 0.25 \\
\hline & IC1554 & J003307.4-321530 & 1814 & 43 & -1 & 10.14 & & & & & \\
\hline 9 & NGC0255 & J004747.3-112807 & 1694 & 15 & 4 & 9.66 & 174 & 10.23 & 5.8 & 3.6 & 0.81 \\
\hline & DDO005 & J004603.4-113020 & 1716 & 5 & 9 & 11.94 & & & & & \\
\hline 10 & NGC0357 & J010321.9-062021 & 2515 & 16 & 0 & 8.43 & 269 & 11.02 & 1.0 & 0.6 & 0.93 \\
\hline & MCG. & J010508.6-061646 & 2533 & 5 & 9 & 12.97 & & & & & \\
\hline 11 & NGC0424 & J011127.7-380500 & 3455 & 7 & 0 & 9.12 & 372 & 11.17 & 5.1 & & 0.74 \\
\hline & NGC0438 & J011334.2-375406 & 3414 & 26 & 3 & 9.99 & & & & & \\
\hline 12 & NGC0428 & J011255.7+005854 & 1287 & 5 & 9 & 9.37 & 65 & 10.07 & 0.5 & & 1.99 \\
\hline & UGC00772 & J011339.4+005228 & 1296 & 6 & 10 & 13.63 & & & & & \\
\hline 13 & NGC0473 & J011955.1+163241 & 2317 & 5 & 0 & 9.53 & 138 & 10.51 & 5.5 & 4.7 & 0.92 \\
\hline & LSBC... & J011947.4+164725 & 2350 & 6 & 10 & 15.65 & & & & & \\
\hline 14 & UGC00903 & J012147.8+173533 & 2697 & 7 & 4 & 9.38 & 355 & 10.70 & 1.4 & 0.6 & 0.40 \\
\hline & UGC00883 & J012101.0+170424 & 2684 & 5 & 10 & 13.63 & & & & & \\
\hline 15 & UGC00964 & J012435.1+074316 & 2884 & 6 & 3 & 11.89 & 220 & 9.89 & 41.7 & 34.9 & 0.41 \\
\hline & VV730... & J012319.3+074747 & 2849 & 8 & 10 & 12.88 & & & & & \\
\hline 16 & LSBC... & J013029.0+024955 & 2240 & 10 & 5 & 13.57 & 60 & 9.09 & 9.1 & & 0.11 \\
\hline & UGC01075 & J013002.5+025109 & 2227 & 6 & 8 & 13.93 & & & & & \\
\hline 17 & NGC0578 & J013029.1-224002 & 1645 & 5 & 5 & 8.58 & 44 & 10.57 & 9.3 & & 0.47 \\
\hline & 2MASX... & J013011.9-224545 & 1563 & 74 & 4 & 12.80 & & & & & \\
\hline 18 & NGC0573 & J013049.3+411526 & 3028 & 5 & 5 & 11.07 & 235 & $10.26 \mid$ & 4.9 & 3.4 & 1.94 \\
\hline
\end{tabular}


Table I: Table. (Contd.)

\begin{tabular}{|c|c|c|c|c|c|c|c|c|c|c|c|}
\hline \multirow[t]{2}{*}{ No. } & Name & $\mathrm{J} 2000.0$ & $\begin{array}{l}V_{L G} \\
\mathrm{~km}\end{array}$ & \pm & $\mathrm{T}$ & $\begin{array}{r}K \\
\mathrm{mag}\end{array}$ & $\begin{array}{l}R_{\perp} \\
\mathrm{kpc}\end{array}$ & $\begin{array}{r}\log L \\
L_{\odot}\end{array}$ & $\begin{array}{r}\frac{M}{L_{K}} \\
\odot\end{array}$ & $\begin{array}{r}\frac{M^{c}}{L_{K}} \\
\odot\end{array}$ & $\log (I I)$ \\
\hline & UGC01070 & $012959.9+405826$ & 3046 & 5 & 6 & 12.12 & & & & & \\
\hline \multirow[t]{2}{*}{19} & UGC01102... & J013229.6+043607 & 2092 & 29 & 6 & 11.30 & 30 & 9.74 & 6.9 & & 0.06 \\
\hline & UGC01105 & J013239.9+043830 & 2125 & 43 & 10 & 14.26 & & & & & \\
\hline \multirow[t]{2}{*}{20} & NGC0613 & J013418.2-292506 & 1470 & 8 & 4 & 7.02 & 503 & 11.12 & 4.3 & 3.3 & 0.68 \\
\hline & ESO413-007 & J012759.3-290512 & 1501 & 8 & -1 & 12.73 & & & & & \\
\hline \multirow[t]{2}{*}{21} & NGC0632 & J013717.5+055240 & 3301 & 12 & -2 & 10.08 & 496 & 10.58 & 113.2 & & 0.38 \\
\hline & UGC01137 & J013512.8+053022 & 3216 & 75 & 10 & 15.22 & & & & & \\
\hline \multirow[t]{2}{*}{22} & NGC0645 & J014008.7+054336 & 3441 & 5 & 3 & 10.15 & 112 & 10.66 & 8.1 & 6.7 & 0.49 \\
\hline & UGC01172 & J013938.5+054658 & 3389 & 12 & 8 & 12.13 & & & & & \\
\hline \multirow[t]{2}{*}{23} & NGC0676 & J014857.3+055427 & 1630 & 6 & 1 & 9.04 & 180 & 10.68 & 14.1 & 13.5 & 0.39 \\
\hline & NGC0693 & J015030.9+060843 & 1686 & 6 & 0 & 9.21 & & & & & \\
\hline \multirow[t]{2}{*}{24} & NGC0723 & J015345.7-234528 & 1486 & 5 & 4 & 10.30 & 227 & 9.81 & 26.1 & 9.9 & 1.30 \\
\hline & ESO477-012 & J015345.7-: & 1460 & 10 & 10 & 14.27 & & & & & \\
\hline \multirow[t]{2}{*}{25} & NGC0779 & J015942.3-055747 & 1461 & 5 & 3 & 8.09 & 421 & 10.68 & 13.6 & 12.6 & 1.30 \\
\hline & UGCA024 & J020431.4-0 & 1425 & 5 & 9 & 12.35 & & & & & \\
\hline \multirow[t]{2}{*}{26} & NGC0821 & J020821.1+105942 & 1862 & 18 & -5 & 7.86 & 537 & 10.98 & 0.0 & & 0.91 \\
\hline & kkh008 & J021227.4+101959 & 1861 & 5 & 10 & 13.83 & & & & & \\
\hline \multirow[t]{2}{*}{27} & NGC0851 & J021112.1+034647 & 3208 & 38 & -1 & 11.02 & 59 & 10.43 & 62.3 & 53.0 & 1.18 \\
\hline & IC0211 & $\mathrm{J} 021108.0+0$ & 3363 & 23 & 6 & 11.47 & & & & & \\
\hline \multirow[t]{2}{*}{28} & NGC0853 & J021141.2-091822 & 1615 & 34 & 9 & 10.44 & 243 & 9.86 & 3.5 & & 1.01 \\
\hline & MRK1025 & J020959.7-085011 & 1624 & 14 & 9 & 13.15 & & & & & \\
\hline \multirow[t]{2}{*}{29} & NGC0865 & J021615.1+283559 & 3175 & 8 & 5 & 10.22 & 304 & 10.51 & 0.3 & & 2.47 \\
\hline & UGC01753 & J021631.8+281213 & 3170 & 6 & 10 & 14.17 & & & & & \\
\hline \multirow[t]{2}{*}{30} & NGC0895 & J022136.5-053117 & 2338 & 5 & 6 & 9.40 & 22 & 10.59 & 8.2 & & 0.14 \\
\hline & NGC0895a & J022145.2-053208 & 2448 & 75 & 9 & 13.86 & & & & & \\
\hline \multirow[t]{2}{*}{31} & NGC0922 & J022504.4-244717 & 3052 & 9 & 6 & 10.02 & 101 & 10.59 & 17.3 & & 1.90 \\
\hline & $2 M A S X \ldots$ & J022430.0-244444 & 3126 & 74 & 4 & 12.99 & & & & & \\
\hline \multirow[t]{2}{*}{32} & NGC0986 & J023334.4-390242 & 1876 & 36 & 2 & 7.77 & 177 & 11.04 & 4.9 & & 1.05 \\
\hline & ESO299-011 & J023534.7-390131 & 1926 & 74 & 5 & 12.54 & & & & & \\
\hline \multirow[t]{2}{*}{33} & IC0239 & J023627.9+385812 & 1096 & 5 & 6 & 8.75 & 238 & 10.16 & 0.1 & & 0.47 \\
\hline & NGC1023C & J024039.6+392247 & 1094 & 5 & 10 & 14.74 & & & & & \\
\hline \multirow[t]{2}{*}{34} & NGC1090 & J024633.9-001449 & 2809 & 5 & 4 & 9.19 & 389 & 10.80 & 54.7 & 43.1 & 0.54 \\
\hline & UGCA042 & J024852.7-002103 & 2722 & 20 & 10 & 14.03 & & & & & \\
\hline \multirow[t]{2}{*}{35} & NGC1140 & J025433.6-100140 & 1508 & 5 & 9 & 10.49 & 204 & 9.77 & 0.0 & & 0.30 \\
\hline & $6 \mathrm{dF} \ldots$ & J025333.5-103208 & 1509 & 74 & 10 & 13.54 & & & & & \\
\hline \multirow[t]{2}{*}{36} & MCG... & J030031.8-154411 & 1506 & 55 & 7 & 10.77 & 143 & 9.74 & 0.0 & & 0.63 \\
\hline & $2 \mathrm{MASX} \ldots$ & J030042.9-160752 & 1507 & 29 & 9 & 12.11 & & & & & \\
\hline 37 & NGC1172 & J030136.1-145012 & 1645 & 8 & -4 & 9.19 & 239 & 10.36 & 5.1 & & 0.17 \\
\hline
\end{tabular}


Table I: Table. (Contd.)

\begin{tabular}{|c|c|c|c|c|c|c|c|c|c|c|c|}
\hline \multirow[t]{2}{*}{ No. } & Name & J2000.0 & $\begin{array}{l}V_{L G} \\
\mathrm{~km} \mathrm{~s}\end{array}$ & \pm & $\mathrm{T}$ & $\begin{array}{r}K \\
\mathrm{mag}\end{array}$ & $\begin{array}{l}R_{\perp} \\
\mathrm{kpc}\end{array}$ & $\begin{array}{r}\log L \\
L_{\odot}\end{array}$ & $\begin{array}{r}\frac{M}{L_{K}} \\
\odot\end{array}$ & $\begin{array}{r}\frac{M^{c}}{L_{K}} \\
\odot\end{array}$ & $\log (I I)$ \\
\hline & IRAS. & J030307.5-151932 & 1625 & 25 & 9 & 12.16 & \multirow{3}{*}{297} & \multirow{3}{*}{10.11} & \multirow{3}{*}{6.2} & & \multirow{3}{*}{2.94} \\
\hline \multirow[t]{2}{*}{38} & UGC02497 & J030207.7+290623 & 3265 & 5 & 8 & 11.31 & & & & & \\
\hline & UGC02488 & J030143.2+284413 & 3280 & 8 & 10 & 14.46 & & & & & \\
\hline \multirow[t]{2}{*}{39} & NGC1196 & J030335.2-120435 & 3371 & 22 & -2 & 9.60 & \multirow[t]{2}{*}{111} & \multirow[t]{2}{*}{10.90} & \multirow[t]{2}{*}{25.4} & \multirow[t]{2}{*}{18.5} & \multirow[t]{2}{*}{0.62} \\
\hline & IC0285 & J030406.2-120056 & 3246 & 35 & 3 & 10.87 & & & & & \\
\hline \multirow[t]{2}{*}{40} & NGC1201 & J030408.0-260411 & 1609 & 20 & -3 & 7.67 & \multirow[t]{2}{*}{312} & \multirow[t]{2}{*}{10.95} & \multirow[t]{2}{*}{9.8} & \multirow[t]{2}{*}{9.3} & \multirow[t]{2}{*}{0.26} \\
\hline & ESO480-025 & J030350.5-251620 & 1657 & 5 & 9 & 12.61 & & & & & \\
\hline \multirow[t]{2}{*}{41} & LCRS... & J031049.7-414757 & 1253 & 74 & 9 & 12.92 & \multirow[t]{2}{*}{41} & \multirow[t]{2}{*}{8.76} & \multirow[t]{2}{*}{379.6} & & \multirow[t]{2}{*}{0.24} \\
\hline & LCRS... & J031059.2-413940 & 1186 & 26 & 9 & 13.75 & & & & & \\
\hline \multirow[t]{2}{*}{42} & NGC1253 & J031409.0-024923 & 1723 & 6 & 6 & 9.23 & 27 & 10.42 & 16.7 & 16.4 & 1.23 \\
\hline & NGC1253A & J031423.3-024803 & 1840 & 8 & 9 & 12.45 & & & & & \\
\hline 43 & 2MASX.. & J031729.7-080843 & 2054 & 26 & 5 & 13.05 & 173 & 9.25 & 14.8 & & 1.72 \\
\hline & SDSS... & J031829.0-075331 & 2065 & 5 & 8 & 13.25 & & & & & \\
\hline 44 & NGC1320 & J032448.7-030232 & 2719 & 45 & 1 & 9.34 & 17 & 10.90 & 0.1 & & 0.30 \\
\hline & NGC1321 & J032448.6-030056 & 2701 & 30 & 1 & 10.01 & & & & & \\
\hline 45 & UGCA071 & J032524.7-161416 & 1827 & 9 & 7 & 11.27 & 39 & 9.63 & 1.4 & 0.2 & 0.54 \\
\hline & MCG... & J032512.1-160950 & 1816 & 5 & 10 & 13.95 & & & & & \\
\hline 46 & ESO548-025 & J032900.7-220848 & 1715 & 71 & 1 & 11.29 & 84 & 9.79 & 5.3 & & 0.17 \\
\hline & NGC1347 & J032941.8-221645 & 1697 & 9 & 5 & 11.53 & & & & & \\
\hline 47 & IC1970 & J033631.5-435725 & 1085 & 5 & 3 & 9.08 & 103 & 10.06 & 1.0 & & 0.23 \\
\hline & ESO249-008 & J033718.9-433510 & 1094 & 9 & 9 & 12.06 & & & & & \\
\hline 48 & NGC1390 & J033752.2-190030 & 1142 & 12 & 6 & 11.52 & 171 & 9.20 & 0.0 & & 0.57 \\
\hline & ESO548-065 & J034002.7-192160 & 1142 & 34 & 8 & 12.87 & & & & & \\
\hline 49 & NGC1412 & J034029.4-265144 & 1686 & 12 & -2 & 9.63 & 35 & 10.20 & 4.2 & & 0.24 \\
\hline & ESO482-032 & J034041.4-264711 & 1645 & 22 & 7 & 12.66 & & & & & \\
\hline 50 & NGC1416 & J034102.9-224309 & 2077 & 24 & -5 & 10.53 & 133 & 10.05 & 59.9 & & 0.03 \\
\hline & $2 \mathrm{MASX} \ldots$ & J034127.1-222823 & 2143 & 75 & -4 & 13.42 & & & & & \\
\hline 51 & NGC1421 & J034229.3-132917 & 2033 & 9 & 4 & 8.37 & 288 & 11.02 & 19.7 & 19.1 & 0.34 \\
\hline & MCG... & J034256.1-125459 & 2111 & 5 & 5 & 9.37 & & & & & \\
\hline 52 & NGC1440 & J034502.9-181558 & 1458 & 27 & -2 & 8.16 & 373 & 10.65 & 1.0 & & 0.12 \\
\hline & ESO549-007 & J034411.5-191910 & 1448 & 11 & 9 & 12.41 & & & & & \\
\hline 53 & IC0334 & J034517.1+763818 & 2762 & 7 & 2 & 7.62 & 963 & 11.41 & 5.2 & 3.8 & 0.58 \\
\hline & HFLLZOAG... & J033724.9+751500 & 2727 & 9 & 10 & 14.11 & & & & & \\
\hline 54 & UGC02906 & J040101.0+740502 & 2837 & 28 & 3 & 8.95 & 160 & 10.91 & 4.6 & 3.8 & 0.26 \\
\hline & HFLLZOAG... & J040330.7+741503 & 2793 & 9 & 10 & 14.76 & & & & & \\
\hline 55 & NGC1527 & J040824.1-475349 & 815 & 38 & -3 & 7.63 & 351 & 10.36 & 2.0 & & 0.21 \\
\hline & AM0358-465 & J035956.4-464705 & 804 & 9 & 5 & 12.12 & & & & & \\
\hline 56 & NGC1533 & J040951.8-560706 & 582 & 20 & -2 & 7.62 & 24 & 10.01 & 16.8 & & 0.56 \\
\hline
\end{tabular}


Table I: Table. (Contd.)

\begin{tabular}{|c|c|c|c|c|c|c|c|c|c|c|c|}
\hline No. & Name & J2000.0 & \begin{tabular}{|c|}
$V_{L G}$ \\
$\mathrm{~km} \mathrm{~s}$
\end{tabular} & \begin{tabular}{|c|} 
\pm \\
\end{tabular} & $\mathrm{T}$ & $\begin{array}{r}K \\
\mathrm{mag}\end{array}$ & $\begin{array}{l}R_{\perp} \\
\mathrm{kpc}\end{array}$ & $\begin{array}{r}\log L \\
L_{\odot}\end{array}$ & $\begin{array}{r}\frac{M}{L_{K}} \\
\odot\end{array}$ & $\begin{array}{r}\frac{M^{c}}{L_{K}} \\
\odot\end{array}$ & $\log (I I)$ \\
\hline & IC2038 & J040853.8-555922 & 505 & 52 & 7 & 14.10 & \multirow{3}{*}{249} & \multirow{3}{*}{10.44} & \multirow{3}{*}{0.8} & & \multirow{3}{*}{0.24} \\
\hline \multirow[t]{2}{*}{57} & NGC1559 & J041735.8-624701 & 1072 & 6 & 6 & 8.01 & & & & & \\
\hline & ESO084-015 & J042211.8-633640 & 1064 & 20 & 9 & 12.91 & & & & & \\
\hline \multirow[t]{2}{*}{58} & IC2059 & J042026.3-314328 & 2653 & 27 & -2 & 9.89 & \multirow[t]{2}{*}{458} & \multirow[t]{2}{*}{10.57} & \multirow[t]{2}{*}{25.6} & 15.5 & \multirow[t]{2}{*}{0.72} \\
\hline & ESO420-015 & J041741.9-311730 & 2611 & 5 & 10 & 11.32 & & & & & \\
\hline \multirow[t]{2}{*}{59} & ESO550-024 & J042113.6-215046 & 782 & 6 & 7 & 10.06 & \multirow[t]{2}{*}{121} & \multirow[t]{2}{*}{9.36} & \multirow[t]{2}{*}{0.6} & & \multirow[t]{2}{*}{0.82} \\
\hline & ESO550-023 & J042012.6-211439 & 785 & 9 & 8 & 14.63 & & & & & \\
\hline \multirow[t]{2}{*}{60} & NGC1530 & J042327.1+751744 & 2676 & 10 & 3 & 8.23 & \multirow[t]{2}{*}{874} & \multirow[t]{2}{*}{11.29} & \multirow[t]{2}{*}{1.1} & 0.1 & \multirow[t]{2}{*}{0.48} \\
\hline & IC0381 & J044428.5+753823 & 2690 & 5 & 4 & 9.30 & & & & & \\
\hline \multirow[t]{2}{*}{61} & NGC1638 & J044136.5-014833 & 3235 & 29 & -2 & 9.20 & \multirow[t]{2}{*}{285} & \multirow[t]{2}{*}{10.95} & \multirow[t]{2}{*}{10.7} & 9.8 & 0.22 \\
\hline & UGC03127 & J044025.4-020127 & 3289 & 5 & 7 & 12.62 & & & & & \\
\hline 62 & MCG.. & J044834.4-035202 & 2690 & 5 & -1 & 10.25 & 326 & 10.55 & 16.7 & & 0.01 \\
\hline & MCG... & J044822.5-032160 & 2729 & 35 & 4 & 10.86 & & & & & \\
\hline 63 & UGC03180 & J045024.2+084247 & 3384 & 33 & 1 & 10.47 & 306 & 10.69 & 51.7 & 16.7 & 2.16 \\
\hline & UGC03188 & J045149.2+085038 & 3467 & 35 & 8 & 10.92 & & & & & \\
\hline 64 & ESO119-016 & J045129.2-613903 & 739 & 10 & 10 & 12.58 & 74 & 8.37 & 13.9 & & 0.08 \\
\hline & SGC. & J045455.4-613353 & 745 & 9 & 10 & 14.30 & & & & & \\
\hline 65 & 2MASX. & J045500.2-371535 & 2105 & 74 & -1 & 12.32 & 34 & 9.58 & 44.0 & & 0.62 \\
\hline & ESO361-019 & J045453.7-371918 & 2170 & 33 & 7 & 12.53 & & & & & \\
\hline 66 & 2MASX. & J050016.8+711208 & 1422 & 38 & 4 & 11.98 & 23 & 9.13 & 0.5 & & 0.31 \\
\hline & UGC03212 & J050102.2+711033 & 1418 & 6 & 10 & 14.73 & & & & & \\
\hline 67 & ESO486-021 & J050319.7-252523 & 683 & 21 & 3 & 11.42 & 14 & 8.72 & 8.6 & & 1.01 \\
\hline & ESO486-015 & J050300.7-252803 & 666 & 75 & 10 & 13.72 & & & & & \\
\hline 68 & NGC1784 & J050527.1-115218 & 2192 & 5 & 5 & 8.47 & 294 & 10.91 & 9.3 & 8.6 & 0.71 \\
\hline & FGC0523 & J050732.0-113906 & 2239 & 6 & 7 & 11.76 & & & & & \\
\hline 69 & UGCA102 & J051048.1-024054 & 2740 & 16 & 3 & 11.92 & 51 & 9.75 & 15.4 & & 1.91 \\
\hline & IIZw 033B & J051045.3-024531 & 2778 & 75 & 10 & 14.13 & & & & & \\
\hline 70 & ESO362-009 & J051159.3-325821 & 737 & 5 & 8 & 10.40 & 57 & 9.18 & 11.5 & 7.0 & 0.30 \\
\hline & ESO362-007 & J051028.3-330109 & 753 & 5 & 10 & 14.65 & & & & & \\
\hline 71 & NGC1924 & J052801.9-051839 & 2423 & 5 & 4 & 9.30 & 485 & 10.66 & 0.2 & & 1.81 \\
\hline & MCG... & J052709.2-060705 & 2427 & 74 & 10 & 12.36 & & & & & \\
\hline 72 & ESO487-020 & J053223.8-251355 & 1750 & 74 & 7 & 13.19 & 130 & 8.93 & 779.5 & & 0.35 \\
\hline & AM0530-245 & J053246.5-245533 & 1684 & 74 & 10 & 14.16 & & & & & \\
\hline 73 & NGC1954 & J053248.3-140346 & 2973 & 5 & 4 & 9.05 & 116 & $11.19 \mid$ & 48.5 & 47.8 & 0.29 \\
\hline & IC2132 & J053228.7-135538 & 3205 & 18 & 1 & 9.37 & & & & & \\
\hline 74 & MCG... & J053653.2-151215 & 3087 & 9 & 0 & 9.87 & 122 & 10.65 & 25.0 & 23.2 & 0.29 \\
\hline & 2MASX & J053612.6-151438 & 2999 & 12 & 5 & 12.33 & & & & & \\
\hline 75 & ESO554-027 & J054306.1-203117 & 2839 & 33 & -1 & 11.38 & 103 & 10.13 & 111.5 & & 1.03 \\
\hline
\end{tabular}


Table I: Table. (Contd.)

\begin{tabular}{|c|c|c|c|c|c|c|c|c|c|c|c|}
\hline No. & Name & J2000.0 & $\begin{array}{l}V_{L G} \\
\mathrm{~km} \mathrm{~s}\end{array}$ & \begin{tabular}{|l|} 
\pm \\
-1
\end{tabular} & $\mathrm{~T}$ & $\begin{array}{r}K \\
\mathrm{mag}\end{array}$ & $\begin{array}{l}R_{\perp} \\
\mathrm{kpc}\end{array}$ & $\begin{array}{r}\log L \\
L_{\odot}\end{array}$ & $\begin{array}{r}\frac{M}{L_{K}} \\
\odot\end{array}$ & $\begin{array}{r}\frac{M^{c}}{L_{K}} \\
\odot\end{array}$ & $\log (I I)$ \\
\hline \multirow{3}{*}{76} & 2MASX. & J054305.9-204014 & 2950 & 74 & 0 & 12.14 & \multirow{3}{*}{313} & \multirow{3}{*}{10.60} & \multirow{3}{*}{2.4} & & \multirow{3}{*}{0.57} \\
\hline & NGC2076 & J054646.7-164708 & 1982 & 8 & -1 & 8.94 & & & & & \\
\hline & HIPASS... & J054423.6-162652 & 1966 & 25 & 8 & 13.72 & & & & & \\
\hline \multirow[t]{2}{*}{77} & NGC2104 & J054704.7-513311 & 914 & 10 & 9 & 10.56 & \multirow[t]{2}{*}{121} & \multirow[t]{2}{*}{9.34} & \multirow[t]{2}{*}{50.6} & 38.4 & \multirow[t]{2}{*}{1.13} \\
\hline & NGC2101 & J054624.2-520519 & 942 & 6 & 10 & 13.01 & & & & & \\
\hline \multirow[t]{2}{*}{78} & NGC2106 & J055046.6-213402 & 1724 & 6 & -2 & 9.11 & \multirow[t]{2}{*}{407} & \multirow[t]{2}{*}{10.42} & \multirow[t]{2}{*}{10.5} & 4.7 & \multirow[t]{2}{*}{0.83} \\
\hline & ESO555-010 & J055257.5-204246 & 1700 & 9 & 9 & 12.91 & & & & & \\
\hline \multirow[t]{2}{*}{79} & IC0438 & J055300.1-175234 & 2939 & 6 & 5 & 9.68 & \multirow[t]{2}{*}{91} & \multirow[t]{2}{*}{10.76} & \multirow[t]{2}{*}{0.2} & & \multirow[t]{2}{*}{0.05} \\
\hline & IC2151 & J055236.4-174714 & 2930 & 10 & 4. & 11.04 & & & & & \\
\hline \multirow[t]{2}{*}{80} & IC2153 & J060004.2-335512 & 2617 & 15 & 5. & 11.25 & \multirow[t]{2}{*}{3} & \multirow[t]{2}{*}{10.15} & 0.1 & & 0.61 \\
\hline & IC2153.. & J060005.4-335505 & 2599 & 28 & 5. & 11.60 & & & & & \\
\hline 81 & IC0441 & J060242.6-122957 & 2047 & 5 & 5. & 10.74 & 232 & 10.20 & 2.2 & 0.4 & 1.68 \\
\hline & MCG... & J060434.9-123729 & 2058 & 5 & 3 & 10.82 & & & & & \\
\hline 82 & ESO 425-014 & J061302.7-274347 & 2718 & 28 & -3 & 9.38 & 595 & 10.75 & 32.1 & & 1.05 \\
\hline & ESO $425-010$ & J060857.3-274812 & 2769 & 56 & 8 & 11.92 & & & & & \\
\hline 83 & NGC2211 & J061830.4-183214 & 1784 & 20 & -2 & 9.36 & 12 & 10.39 & 0.7 & 0.3 & 0.15 \\
\hline & NGC2212 & J061835.8-183110 & 1818 & 11 & 6 . & 12.06 & & & & & \\
\hline 84 & NGC2221 & J062015.7-573442 & 2262 & 50 & 3 & 10.05 & 26 & 10.40 & 6.1 & 2.8 & 0.49 \\
\hline & NGC2222 & J062016.1-573151 & 2332 & 11 & 4 & 11.39 & & & & & \\
\hline 85 & UGC03445 & J062132.8+590736 & 3241 & 22 & 2 & 8.60 & 10 & 11.35 & 0.1 & & 0.82 \\
\hline & UGC03446 & J062138.9+590733 & 3291 & 35 & 1 & 9.38 & & & & & \\
\hline 86 & NGC2223 & J062435.9-225018 & 2502 & 19 & 4 & 8.83 & 128 & 10.90 & 60.2 & 19.6 & 0.27 \\
\hline & ESO489-052 & J062520.6-224327 & 2679 & 74 & 4 & 12.45 & & & & & \\
\hline 87 & PGC179437 & J062501.7-372252 & 2607 & 8 & -1 & 12.39 & 19 & 9.75 & 0.3 & & 1.47 \\
\hline & $2 \mathrm{MASX} \ldots$ & J062456.0-372126 & 2616 & 74 & 3 & 12.45 & & & & & \\
\hline 88 & $6 \mathrm{dF} \ldots$ & J062534.5-282716 & 2597 & 74 & -1 & 10.90 & 193 & 10.30 & 101.9 & & 1.11 \\
\hline & ESO426-010 & J062639.3-283952 & 2503 & 6 & 3 & 11.12 & & & & & \\
\hline 89 & ESO122-001 & J064043.2-583128 & 2376 & 30 & 3 & 9.26 & 32 & 10.68 & 6.0 & 4.8 & 1.47 \\
\hline & ESO122-002 & J064046.6-582811 & 2463 & 18 & 5. & 12.09 & & & & & \\
\hline 90 & NGC2273 & J065008.7+605045 & 1968 & 10 & 1 & 8.45 & 382 & 10.78 & 17.8 & & 0.49 \\
\hline & MAILYAN017 & J064639.2+600845 & 1919 & 75 & 10 & 14.02 & & & & & \\
\hline 91 & UGC03509 & J065455.4+853817 & 1865 & 46 & 2 & 11.20 & 77 & 9.65 & 77.8 & 70.9 & 0.44 \\
\hline & UGC03496 & J065036.0+854742 & 1804 & 5 & 10 & 15.04 & & & & & \\
\hline 92 & UGC03647 & J070450.4+563113 & 1488 & 5 & 10 & 12.12 & 44 & 9.28 & 0.7 & & 0.95 \\
\hline & CGCG... & J070359.2+562911 & 1493 & 25 & 10 & 12.73 & & & & & \\
\hline 93 & UGC03657 & J070724.1+711133 & 3427 & 75 & 6 . & 13.50 & 153 & 9.49 & 19.6 & & 0.17 \\
\hline & UGC03644 & J070538.2+710413 & 3409 & 11 & 7 . & 13.87 & & & & & \\
\hline 94 & NGC2337 & J071013.6+442726 & 477 & 5 & 8. & 10.31 & 20 & 8.82 & 5.3 & 1.6 & 0.83 \\
\hline
\end{tabular}


Table I: Table. (Contd.)

\begin{tabular}{|c|c|c|c|c|c|c|c|c|c|c|c|}
\hline No. & Name & J2000.0 & \begin{tabular}{|c|}
$V_{L G}$ \\
$\mathrm{~km} \mathrm{~s}$
\end{tabular} & & $\mathrm{~T}$ & $\begin{array}{r}K \\
\mathrm{mag}\end{array}$ & $\begin{array}{l}R_{\perp} \\
\mathrm{kpc}\end{array}$ & $\begin{array}{r}\log L \\
L_{\odot}\end{array}$ & $\begin{array}{r}\frac{M}{L_{K}} \\
\odot\end{array}$ & $\begin{array}{r}\frac{M^{c}}{L_{K}} \\
\odot\end{array}$ & $\log (I I)$ \\
\hline & UGC03698 & J070918.7+442248 & 465 & 5 & 10 & 14.24 & & & & & \\
\hline 95 & UGC03730... & J071420.6+732850 & 2897 & 18 & 2 & 10.78 & 106 & 10.41 & 6.6 & 2.6 & 0.89 \\
\hline & UGC03705 & J071211.2+732814 & 2860 & 11 & 9 & 11.27 & & & & & \\
\hline 96 & NGC2268 & J071417.4+842256 & 2440 & 5 & 4 & 8.56 & 391 & 10.94 & 41.4 & 40.8 & 0.26 \\
\hline & UGC03522 & J065606.1+845504 & 2352 & 5 & 10 & 12.12 & & & & & \\
\hline 97 & NGC2357 & J071741.0+232124 & 2204 & 5 & 6 & 9.61 & 484 & 10.59 & 11.5 & 10.1 & 0.40 \\
\hline & UGC03751 & J071353.9+230449 & 2232 & 5 & 6 & 10.51 & & & & & \\
\hline 98 & UGC03788 & J071825.9+313340 & 3443 & 7 & 4 & 11.15 & 142 & 10.29 & 0.5 & & 0.68 \\
\hline & UGC03790. & J071833.0+312327 & 3451 & 56 & 7 & 12.73 & & & & & \\
\hline 99 & UGC03789 & J071930.9+592118 & 3396 & 58 & 2 & 9.49 & 60 & 10.88 & 13.6 & 12.5 & 0.18 \\
\hline & UGC03797 & J072003.1+592243 & 3517 & 15 & 6 & 13.40 & & & & & \\
\hline 100 & NGC2276 & J072714.4+854516 & 2632 & 12 & 5 & 9.68 & 111 & 10.70 & 31.3 & 27.4 & 0.53 \\
\hline & UGC03654 & $\mathrm{J} 071747.1+\varepsilon$ & 2523 & 22 & -3 & 10.48 & & & & & \\
\hline 101 & IC2202 & J072754.7-673427 & 3327 & 6 & 4 & 9.40 & 566 & 10.96 & 0.0 & & 0.21 \\
\hline & ESO058-028 & J072034.0-674239 & 3324 & 10 & 7 & 10.97 & & & & & \\
\hline 102 & UGC03864 & J073057.0+723102 & 2737 & 50 & 5 & 12.40 & 5 & 9.51 & 1.0 & & 0.55 \\
\hline & VV141b & J073054.8+723037 & 2714 & 75 & 10 & 15.83 & & & & & \\
\hline 103 & UGC03974 & J074155.4+164809 & 162 & 5 & 10 & 11.40 & 11 & 7.54 & 11.7 & & 1.69 \\
\hline & CGCG... & J074232.0+163340 & 168 & 5 & 10 & 13.11 & & & & & \\
\hline 104 & ESO035-018 & J075504.2-762445 & 1492 & 5 & 5 & 9.76 & 288 & 10.04 & 4.6 & & 0.73 \\
\hline & ESO035-020 & J080318.6-770419 & 1479 & 9 & 9 & 13.15 & & & & & \\
\hline 105 & UGC04159 & J080151.2+612447 & 1703 & 8 & 9 & 11.50 & 37 & 9.60 & 0.0 & & 0.50 \\
\hline & UGC04169 & J080234.1+612253 & 1701 & 5 & 9 & 12.42 & & & & & \\
\hline 106 & UGC04151 & $\mathrm{J} 080418.7+774860$ & 2473 & 5 & 6 & 10.39 & 276 & 10.30 & 3.2 & 1.6 & 0.70 \\
\hline & UGC04066 & J075615.6+780048 & 2487 & 5 & 8 & 12.09 & & & & & \\
\hline 107 & ESO124-014 & J080912.7-613937 & 2708 & 20 & -3 & 9.29 & 231 & 10.93 & 7.7 & & 1.54 \\
\hline & IRAS... & J080852.5-611835 & 2756 & 44 & 3 & 10.00 & & & & & \\
\hline 108 & LCSBS1123P & J081715.9+245357 & 1832 & 5 & 9 & 13.68 & 29 & 8.75 & 41.0 & & 0.88 \\
\hline & KUG0814... & J081721.0+245746 & 1806 & 70 & 9 & 14.99 & & & & & \\
\hline 109 & CGCG... & J081725.4+210950 & 2054 & 9 & 8 & 13.12 & 10 & 9.24 & 5.9 & & 0.46 \\
\hline & CGCG... & J081728.1+211052 & 2024 & 75 & 4 & 13.20 & & & & & \\
\hline 110 & IC2267 & J081801.5+244411 & 1962 & 10 & 6 & 11.84 & 29 & 9.57 & 11.2 & 9.4 & 0.75 \\
\hline & IC2268 & J081806.6+244747 & 1928 & 5 & 9 & 12.90 & & & & & \\
\hline 111 & NGC2549 & J081858.3+574811 & 1154 & 22 & -2 & 8.02 & 127 & 10.51 & 0.5 & & 0.50 \\
\hline & UGC04314 & J081857.8+581547 & 1164 & 7 & 9 & 12.24 & & & & & \\
\hline 112 & $6 \mathrm{dF} \ldots$ & J082142.8-002601 & 1612 & 74 & 10 & 12.88 & 27 & 8.97 & 1.2 & & 1.93 \\
\hline & UGC04358 & J082126.0-002508 & 1606 & 6 & 10 & 14.03 & & & & & \\
\hline 113 & $\mathrm{SAO} 0822+3545$ & J082605.6+353526 & 671 & 50 & 10 & 15.02 & 10 & 7.48 & 133.2 & & 1.57 \\
\hline
\end{tabular}


Table I: Table. (Contd.)

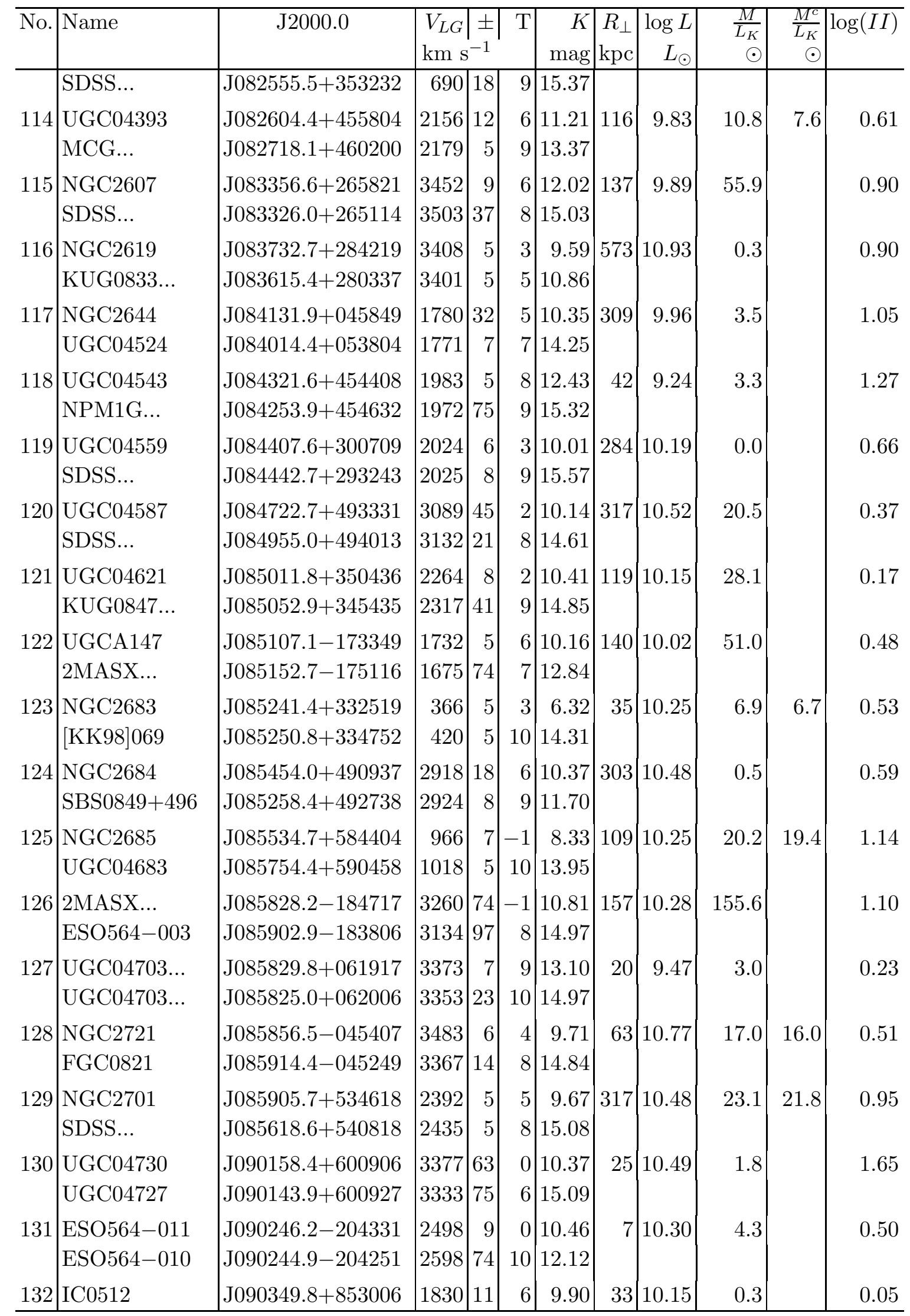


Table I: Table. (Contd.)

\begin{tabular}{|c|c|c|c|c|c|c|c|c|c|c|c|}
\hline No. & Name & $\mathrm{J} 2000.0$ & $\begin{array}{c}V_{L G} \\
\mathrm{~km} \mathrm{~s}\end{array}$ & & $\mathrm{~T}$ & $\begin{array}{r}K \\
\mathrm{mag}\end{array}$ & $\begin{array}{l}R_{\perp} \\
\mathrm{kpc}\end{array}$ & $\begin{array}{r}\log L \\
L_{\odot}\end{array}$ & $\begin{array}{r}\frac{M}{L_{K}} \\
\odot\end{array}$ & $\begin{array}{r}\frac{M^{c}}{L_{K}} \\
\odot\end{array}$ & $\log (I I)$ \\
\hline & UGC04612 & J090018.6+853156 & 1820 & 11 & 9 & 14.33 & \multirow{3}{*}{123} & \multirow{3}{*}{9.16} & \multirow{3}{*}{340.3} & & \multirow{3}{*}{1.80} \\
\hline \multirow[t]{2}{*}{133} & KUG0901... & J090440.1+472415 & 2310 & 35 & 4 & 13.34 & & & & & \\
\hline & SDSS... & J090331.1+473028 & 2368 & 67 & 9 & 14.15 & & & & & \\
\hline \multirow[t]{2}{*}{134} & ESO564-019 & J090527.5-183130 & 1730 & 74 & 4 & 11.30 & \multirow[t]{2}{*}{211} & \multirow[t]{2}{*}{9.81} & \multirow[t]{2}{*}{90.6} & & \multirow[t]{2}{*}{0.48} \\
\hline & NGC2758 & J090531.2-190234 & 1682 & 5 & 4 & 11.40 & & & & & \\
\hline \multirow[t]{2}{*}{135} & NGC2772 & J090741.9-233717 & 3139 & 5 & 3 & 9.33 & \multirow[t]{2}{*}{7} & \multirow[t]{2}{*}{10.86} & \multirow[t]{2}{*}{0.5} & 0.4 & \multirow[t]{2}{*}{0.91} \\
\hline & ESO-LV ... & J090741.1-233749 & 3205 & 15 & 10 & 15.28 & & & & & \\
\hline \multirow[t]{2}{*}{136} & NGC2764 & J090817.5+212636 & 2608 & 5 & -2 & 9.80 & \multirow[t]{2}{*}{$104 \mid$} & \multirow[t]{2}{*}{10.50} & \multirow[t]{2}{*}{1.1} & 0.7 & \multirow[t]{2}{*}{0.88} \\
\hline & LSBC. & J090855.3+212149 & 2591 & 5 & 9 & 14.39 & & & & & \\
\hline \multirow[t]{2}{*}{137} & UGC04809 & J090920.3+204150 & 2907 & 8 & 6 & 11.97 & \multirow[t]{2}{*}{91} & \multirow[t]{2}{*}{9.82} & \multirow[t]{2}{*}{18.3} & 13.3 & 0.91 \\
\hline & MCG... & J090904.6+203452 & 2873 & 9 & 9 & 13.40 & & & & & \\
\hline 138 & CGCG... & J091023.5+192719 & 3007 & 10 & -1 & 11.43 & 44 & 10.02 & 0.0 & & 0.80 \\
\hline & UGC04822 & J091038.5+192823 & 3005 & 5 & 8 & 13.72 & & & & & \\
\hline 139 & UGCA150 & J091048.8-085338 & 1591 & 5 & 3 & 7.69 & 157 & 10.92 & 0.2 & & 0.10 \\
\hline & 2MASX. & J090939.9-083547 & 1600 & 74 & 9 & 13.41 & & & & & \\
\hline 140 & NGC2784 & J091219.5-241021 & 407 & 35 & -2 & 6.24 & 132 & 10.34 & 7.0 & 3.4 & 0.21 \\
\hline & ESO497-017 & J090946.5-230033 & 438 & 11 & 10 & 11.81 & & & & & \\
\hline 141 & IC2445 & J091312.6+314828 & 1859 & 9 & 6 & 12.78 & 65 & 9.08 & 14.2 & 5.4 & 0.58 \\
\hline & SDSS... & J091251.7+314051 & 1844 & 5 & 8 & 14.70 & & & & & \\
\hline 142 & KUG0910. & J091340.9+331930 & 3374 & 42 & 5 & 13.16 & 128 & 9.63 & 0.0 & & 0.85 \\
\hline & UGC04850 & J091304.6+332517 & 3375 & 20 & 8 & 13.43 & & & & & \\
\hline 143 & NGC2785 & J091515.4+405503 & 2728 & 10 & 7 & 9.41 & 81 & 10.69 & 6.5 & 6.2 & 0.21 \\
\hline & SDSS... & J091435.6+405524 & 2671 & 6 & 7 & 14.58 & & & & & \\
\hline 144 & ESO497-029 & J091543.3-234205 & 3134 & 14 & 6 & 11.76 & 113 & 10.03 & 0.0 & & 0.91 \\
\hline & ESO $497-028$ & J091534.4-235053 & 3134 & 74 & 9 & 12.65 & & & & & \\
\hline 145 & NGC2811 & J091611.1-161846 & 2099 & 29 & 1 & 7.96 & 165 & 11.02 & 44.9 & 4.5 & 0.61 \\
\hline & $6 \mathrm{dF} \ldots$ & J091648.3-160022 & 1944 & 74 & 9 & 12.74 & & & & & \\
\hline 146 & NGC2815 & J091619.8-233760 & 2258 & 8 & 3 & 8.20 & 125 & 11.03 & 7.1 & 4.9 & 0.04 \\
\hline & NGC2815:... & J091547.6-232628 & 2330 & 20 & 9 & 14.93 & & & & & \\
\hline 147 & IC0529 & J091832.8+734534 & 2422 & 5 & 5 & 9.47 & 275 & 10.62 & 3.9 & & 0.43 \\
\hline & CGCG... & J091215.0+733539 & 2445 & 18 & 9 & 11.65 & & & & & \\
\hline 148 & NGC2787 & J091918.6+691212 & 838 & 5. & -1 & 7.21 & 187 & 10.51 & 41.0 & 39.8 & 0.28 \\
\hline & UGC04998 & J092511.0+682259 & 761 & 7 & 10 & 13.16 & & & & & \\
\hline 149 & NGC2858 & J092255.0+030925 & 3446 & 32 & 0 & 9.80 & 93 & 10.76 & 0.4 & & 0.21 \\
\hline & $2 \mathrm{MASX} .$. & J092234.1+030501 & 3432 & 36 & -1 & 12.91 & & & & & \\
\hline 150 & NGC2852 & J092314.6+400950 & 1778 & 25 & 1 & 10.09 & 16 & 10.16 & 0.5 & & 0.39 \\
\hline & NGC2853 & J092317.3+401200 & 1758 & 30 & 4 & 11.41 & & & & & \\
\hline 151 & UGC04984 & J092339.7+542900 & 3452 & 63 & 9 & 13.50 & 165 & 9.36 & 318.8 & & 1.61 \\
\hline
\end{tabular}


Table I: Table. (Contd.)

\begin{tabular}{|c|c|c|c|c|c|c|c|c|c|c|c|}
\hline No. & Name & $\mathrm{J} 2000.0$ & $\begin{array}{c}V_{L G} \\
\mathrm{~km} \mathrm{~s}\end{array}$ & $\begin{array}{c} \pm \\
-1\end{array}$ & $\mathrm{~T}$ & $\begin{array}{r}K \\
\mathrm{mag}\end{array}$ & $\begin{array}{l}R_{\perp} \\
\mathrm{kpc}\end{array}$ & $\begin{array}{r}\log L \\
L_{\odot}\end{array}$ & $\begin{array}{r}\frac{M}{L_{K}} \\
\odot\end{array}$ & $\begin{array}{r}\frac{M^{c}}{L_{K}} \\
\odot\end{array}$ & $\log (I I)$ \\
\hline \multirow{3}{*}{152} & SBS0919+545 & J092316.4+541734 & 3513 & 18 & 9 & 15.11 & \multirow{3}{*}{54} & \multirow{3}{*}{10.35} & \multirow{3}{*}{6.6} & \multirow{7}{*}{6.9} & \multirow{3}{*}{0.35} \\
\hline & ESO498-003 & J092336.5-265255 & 2070 & 10 & 3 & 9.73 & & & & & \\
\hline & ESO497-042 & J092312.0-265627 & 2118 & 75 & 5 & 13.39 & & & & & \\
\hline \multirow[t]{2}{*}{153} & NGC2891 & J092656.6-244659 & 2067 & 20 & -3 & 9.52 & \multirow[t]{2}{*}{300} & \multirow[t]{2}{*}{10.61} & \multirow[t]{2}{*}{25.9} & & \multirow[t]{2}{*}{0.46} \\
\hline & ESO498-005 & J092440.7-250534 & 2121 & 25 & 4 & 10.17 & & & & & \\
\hline \multirow[t]{2}{*}{154} & UGC05052 & J093114.0+734838 & 3253 & 19 & 2 & 10.28 & \multirow[t]{2}{*}{313} & \multirow[t]{2}{*}{10.68} & \multirow[t]{2}{*}{66.9} & & \multirow[t]{2}{*}{0.64} \\
\hline & CGCG. & J092540.4+735400 & 3346 & 66 & 3 & 11.11 & & & & & \\
\hline \multirow[t]{2}{*}{155} & MCG... & J093612.4-082604 & 1701 & 59 & -4 & 9.76 & \multirow[t]{2}{*}{175} & \multirow[t]{2}{*}{10.17} & \multirow[t]{2}{*}{6.3} & & \multirow[t]{2}{*}{0.74} \\
\hline & MCG... & J093520.4-084836 & 1680 & 6 & 9 & 12.46 & & & & & \\
\hline \multirow[t]{2}{*}{156} & NGC2979 & J094308.7-102260 & 2451 & 31 & 1 & 9.50 & \multirow[t]{2}{*}{257} & \multirow[t]{2}{*}{10.70} & 0.0 & & 0.98 \\
\hline & MCG... & J094317.9-095644 & 2449 & 5 & 7 & 10.62 & & & & & \\
\hline 157 & NGC2983 & J094341.1-202838 & 1767 & 45 & -1 & 8.52 & 94 & 10.66 & 5.9 & & 0.41 \\
\hline & $6 \mathrm{dF} \ldots$ & J094356.9-204142 & 1717 & 74 & 8 & 13.05 & & & & & \\
\hline 158 & NGC3020 & J095006.6+124849 & 1283 & 5 & 6 & 10.67 & 30 & 9.74 & 3.6 & 3.0 & 1.23 \\
\hline & NGC3024 & J095027.4+124556 & 1260 & 5 & 7 & 11.17 & & & & & \\
\hline 159 & UGC05272 & J095022.4+312916 & 460 & 5 & 10 & 12.01 & 4 & 8.14 & 2.8 & & 0.86 \\
\hline & UGC05272b & J095019.4+312721 & 469 & 13 & 10 & 15.32 & & & & & \\
\hline 160 & NGC3032 & J095208.2+291410 & 1472 & 12 & -2 & 9.64 & 70 & 10.09 & 54.0 & 52.6 & 0.46 \\
\hline & KUG0950. & J095257.6+291837 & 1561 & 7 & 8 & 15.77 & & & & & \\
\hline 161 & NGC3052 & J095427.9-183820 & 3502 & 5 & 5 & 9.41 & 650 & 11.09 & 36.6 & 35.7 & 0.10 \\
\hline & ESO566-019 & J095113.4-182833 & 3425 & 7 & 6 & 10.04 & & & & & \\
\hline 162 & NGC3044 & J095340.9+013447 & 1115 & 34 & 6 & 8.97 & 29 & 10.11 & 7.4 & 3.2 & 0.72 \\
\hline & APMUKS... & J095404.6+013224 & 1168 & 20 & 10 & 17.94 & & & & & \\
\hline 163 & NGC3055 & J095518.1+041612 & 1626 & 18 & 5 & 9.48 & 82 & 10.22 & 0.2 & & 0.24 \\
\hline & SDSS... & J095435.7+042308 & 1620 & 66 & 9 & 14.81 & & & & & \\
\hline 164 & NGC3043 & J095614.8+591826 & 3082 & 15 & 5 & 10.45 & 256 & 10.40 & 0.1 & & 0.45 \\
\hline & SBS0953+592 & J095722.4+585929 & 3085 & 5 & 9 & 14.46 & & & & & \\
\hline 165 & NGC3065 & $\mathrm{J} 100155.3+721013$ & 2160 & 10 & 0 & 8.97 & 26 & 10.83 & 2.0 & 0.9 & 0.45 \\
\hline & NGC3066 & $\mathrm{J} 100211.1+720731$ & 2227 & 29 & 4 & 9.92 & & & & & \\
\hline 166 & UGC05403 & J100235.5+191037 & 1958 & 10 & 1 & 10.32 & 67 & 10.03 & 47.9 & 47.1 & 1.58 \\
\hline & UGC05401 & $\mathrm{J} 100231.2+190158$ & 1877 & 5 & 9 & 14.52 & & & & & \\
\hline 167 & NGC3107 & $\mathrm{J} 100422.5+133717$ & 2652 & 7 & 4 & 10.32 & 477 & 10.44 & 0.1 & & 0.15 \\
\hline & CGCG... & $\mathrm{J} 100551.2+125741$ & 2653 & 5 & 1 & 11.40 & & & & & \\
\hline 168 & NGC3124 & J100639.9-191318 & 3285 & 5 & 4 & 9.07 & 152 & 10.99 & 13.6 & 7.0 & 0.13 \\
\hline & $2 M A S X \ldots$ & J100711.1-190405 & 3199 & 30 & 4 & 13.49 & & & & & \\
\hline 169 & NGC3118 & $\mathrm{J} 100711.6+330140$ & 1291 & 5 & 4 & 11.66 & 51 & 9.25 & 56.1 & & 0.71 \\
\hline & UGC05446 & J100630.9+325647 & 1332 & 42 & 6 & 13.19 & & & & & \\
\hline 170 & ESO567-018 & J100726.2-212836 & 3307 & 30 & 3 & 10.86 & 346 & 10.39 & 28.7 & & 0.50 \\
\hline
\end{tabular}


Table I: Table. (Contd.)

\begin{tabular}{|c|c|c|c|c|c|c|c|c|c|c|c|}
\hline No. & Name & J2000.0 & $\begin{array}{l}V_{L G} \\
\mathrm{~km}\end{array}$ & $| \pm|$ & $\mathrm{T}$ & $\begin{array}{r}K \\
\mathrm{mag}\end{array}$ & \begin{tabular}{|l|}
$R_{\perp}$ \\
$\mathrm{kpc}$
\end{tabular} & $\begin{array}{r}\log L \\
L_{\odot}\end{array}$ & $\begin{array}{r}\frac{M}{L_{K}} \\
\odot\end{array}$ & $\begin{array}{r}\frac{M^{c}}{L_{K}} \\
\odot\end{array}$ & $\log (I I)$ \\
\hline & ESO567-014 & $100548.5-211547$ & 3349 & 74 & 7 & 12.28 & \multirow{3}{*}{242} & \multirow{3}{*}{10.08} & \multirow{3}{*}{15.1} & \multirow{3}{*}{9.5} & \multirow{3}{*}{0.40} \\
\hline \multirow[t]{2}{*}{171} & IC0591 & $\mathrm{J} 100727.7+121628$ & 2659 & 12 & 4 & 11.25 & & & & & \\
\hline & UGC05454 & $\mathrm{J} 100711.0+123905$ & 2634 & 5 & 9 & 12.18 & & & & & \\
\hline \multirow[t]{2}{*}{172} & CGCG. & J100757.1+131339 & 2605 & 11 & 6 & 12.34 & \multirow[t]{2}{*}{96} & \multirow[t]{2}{*}{9.54} & \multirow[t]{2}{*}{12.5} & \multirow[t]{2}{*}{7.5} & \multirow[t]{2}{*}{0.07} \\
\hline & SDSS... & $\mathrm{J} 100733.2+130624$ & 2585 & 5 & 9 & 14.28 & & & & & \\
\hline \multirow[t]{2}{*}{173} & MCG... & J100903.3-111360 & 3216 & 33 & -2 & 9.78 & \multirow[t]{2}{*}{37} & \multirow[t]{2}{*}{10.80} & \multirow[t]{2}{*}{42.7} & \multirow[t]{4}{*}{29.2} & \multirow[t]{2}{*}{0.03} \\
\hline & $2 M A S X \ldots$ & J100906.5-111119 & 3462 & 74 & 0 & 11.61 & & & & & \\
\hline \multirow[t]{2}{*}{174} & IC0598 & $\mathrm{J} 101248.6+430844$ & 2263 & 13 & 2 & 10.14 & \multirow[t]{2}{*}{168} & \multirow[t]{2}{*}{10.24} & \multirow[t]{2}{*}{20.2} & & \multirow[t]{2}{*}{1.25} \\
\hline & KUG1008... & J101152.9+432432 & 2222 & 31 & 9 & 14.62 & & & & & \\
\hline \multirow[t]{2}{*}{175} & NGC3153 & $\mathrm{J} 101250.5+124000$ & 2656 & 5 & 6 & 10.60 & 354 & 10.20 & 10.2 & & 0.65 \\
\hline & SDSS... & J101033.9+123540 & 2636 & 11 & 8 & 14.98 & & & & & \\
\hline 176 & IC2558 & J101444.1-342019 & 2292 & 35 & 7 & 11.09 & 180 & 9.91 & 3.1 & & 0.05 \\
\hline & ESO375-003 & 340653 & 2303 & 9 & 9 & 13.68 & & & & & \\
\hline 177 & ESO500-018 & J101453.7-230302 & 3409 & 27 & -2 & 9.64 & 358 & 10.79 & 73.9 & & 0.25 \\
\hline & $2 \mathrm{MASX} .$. & -232904 & 3305 & 74 & 4 & 13.80 & & & & & \\
\hline 178 & UGC05541 & J101655.4+582342 & 2344 & 5 & 10 & 13.52 & 84 & 9.00 & 46.2 & 36.3 & 0.49 \\
\hline & SDSS... & J101712.2+583225 & 2366 & 5 & 9 & 15.21 & & & & & \\
\hline 179 & IC0600 & J101710.9-032952 & 1082 & 5 & 8 & 11.70 & 4 & 8.97 & 31.4 & 25.9 & 1.37 \\
\hline & LCRS... & J101712.7-032901 & 1003 & 17 & 10 & 14.58 & & & & & \\
\hline 180 & NGC3184 & $\mathrm{J} 101817.0+412528$ & 589 & 5 & 6 & 7.22 & 394 & 10.25 & 1.1 & & 0.09 \\
\hline & NGC3104 & +404525 & 595 & 7 & 10 & 11.19 & & & & & \\
\hline 181 & ESO567-052 & J102008.0-214143 & 3251 & 27 & -2 & 10.83 & 21 & 10.47 & 3.5 & & 0.29 \\
\hline & ESO567-053 & J102008.9-214319 & 3187 & 59 & 4 & 11.43 & & & & & \\
\hline 182 & NGC3206 & $\mathrm{J} 102147.6+565550$ & 1239 & 5 & 6 & 11.15 & 85 & 9.54 & 16.1 & & 0.38 \\
\hline & NGC3220 & $\mathrm{J} 102344.7+570137$ & 1262 & 16 & 6 & 11.59 & & & & & \\
\hline 183 & UGC05646 & $\mathrm{J} 102553.1+142147$ & 1223 & 5 & 5 & 10.94 & 145 & 9.49 & 13.7 & 8.2 & 1.01 \\
\hline & UGC05633 & $\mathrm{J} 102440.1+144526$ & 1239 & 5 & 8 & 12.42 & & & & & \\
\hline 184 & NGC3246 & $\mathrm{J} 102641.8+035143$ & 1957 & 23 & 8 & 10.72 & 301 & 9.90 & 1.5 & & 1.44 \\
\hline & VIIIZw 081 & $\mathrm{~J} 102848.1+041405$ & 1951 & 5 & 9 & 14.15 & & & & & \\
\hline 185 & UGC05707 & $\mathrm{J} 103114.3+430815$ & 2807 & 5 & 6 & 14.03 & 32 & 9.05 & 55.8 & 52.5 & 2.14 \\
\hline & SDSS... & J103118.6+430534 & 2848 & 5 & 9 & 14.80 & & & & & \\
\hline 186 & IC2594 & J103604.2-241923 & 3265 & 20 & -3 & 9.32 & 120 & 11.03 & 0.1 & & 0.08 \\
\hline & ESO501-024 & J103527.2-242303 & 3274 & 28 & -1 & 10.37 & & & & & \\
\hline 187 & NGC3294 & J103616.3+371929 & 1556 & 14 & 5 & 8.38 & 150 & 10.66 & 49.8 & 30.8 & 0.73 \\
\hline & KUG1032... & $\mathrm{J} 103522.8+374018$ & 1669 & 35 & 9 & 12.91 & & & & & \\
\hline 188 & NGC3301 & $\mathrm{J} 103656.0+215256$ & 1240 & 17 & -1 & 8.51 & 160 & 10.47 & 10.9 & 8.7 & 0.22 \\
\hline & NGC3287 & $\mathrm{J} 103447.3+213854$ & 1199 & 5 & 8 & 9.77 & & & & & \\
\hline 189 & NGC3306 & $\mathrm{J} 103710.2+123909$ & 2738 & 5 & 8 & 10.40 & 325 & 10.33 & 18.7 & 15.3 & 0.47 \\
\hline
\end{tabular}


Table I: Table. (Contd.)

\begin{tabular}{|c|c|c|c|c|c|c|c|c|c|c|c|}
\hline No. & Name & J2000.0 & $\begin{array}{l}V_{L G} \\
\mathrm{~km} \mathrm{~s}\end{array}$ & \begin{tabular}{|c|} 
\pm \\
-1
\end{tabular} & $\mathrm{~T}$ & $\begin{array}{r}K \\
\mathrm{mag}\end{array}$ & $\begin{array}{l}R_{\perp} \\
\mathrm{kpc}\end{array}$ & $\begin{array}{r}\log L \\
L_{\odot}\end{array}$ & $\begin{array}{r}\frac{M}{L_{K}} \\
\odot\end{array}$ & $\begin{array}{r}\frac{M^{c}}{L_{K}} \\
\odot\end{array}$ & $\log (I I)$ \\
\hline & CGCG. & J103723.4+120924 & 2706 & 7 & 8 & 13.01 & \multirow{3}{*}{85} & \multirow{3}{*}{10.70} & \multirow{3}{*}{30.9} & & \multirow{3}{*}{0.40} \\
\hline \multirow[t]{2}{*}{190} & IC0630 & J103833.8-071015 & 1930 & 10 & 9 & 8.63 & & & & & \\
\hline & MRK1258 & J103800.4-071802 & 1806 & 75 & 9 & 11.84 & & & & & \\
\hline \multirow[t]{2}{*}{191} & NGC3320 & J103936.5+472353 & 2353 & 7 & 4. & 10.09 & \multirow[t]{2}{*}{356} & \multirow[t]{2}{*}{10.30} & \multirow[t]{2}{*}{10.8} & & \multirow[t]{2}{*}{0.78} \\
\hline & SDSS... & $\mathrm{J} 104309.7+471258$ & 2375 & 19 & 7 & 15.00 & & & & & \\
\hline \multirow[t]{2}{*}{192} & $6 \mathrm{dF} \ldots$ & J104011.8-095640 & 2218 & 74 & 6 & 13.43 & \multirow[t]{2}{*}{128} & \multirow[t]{2}{*}{9.07} & \multirow[t]{2}{*}{221.4} & & \multirow[t]{2}{*}{0.90} \\
\hline & $6 \mathrm{dF} \ldots$ & J103930.4-094609 & 2177 & 74 & 9 & 14.18 & & & & & \\
\hline \multirow[t]{2}{*}{193} & AM1039-31: & $\mathrm{J} 104120.7-314855$ & 2388 & 74 & 0 & 10.58 & \multirow[t]{2}{*}{24} & \multirow[t]{2}{*}{10.25} & \multirow[t]{2}{*}{8.1} & & \multirow[t]{2}{*}{0.50} \\
\hline & ESO $437-042$ & J104127.7-314649 & 2317 & 18 & 5. & 11.49 & & & & & \\
\hline \multirow[t]{2}{*}{194} & ESO568-021 & J104115.2-210123 & 3322 & 75 & 3 & 11.15 & \multirow[t]{2}{*}{385} & \multirow[t]{2}{*}{10.26} & \multirow[t]{2}{*}{73.0} & & 0.60 \\
\hline & ESO568-023 & J104248.5-204147 & 3268 & 14 & 7 . & 12.67 & & & & & \\
\hline 195 & ESO569-001 & J104409.1-204809 & 3375 & $37-$ & -2 & 11.02 & 14 & 10.28 & 16.0 & & 0.19 \\
\hline & 2MASX... & J104409 & 3511 & 74 & 5 & 13.80 & & & & & \\
\hline 196 & NGC3329 & $\mathrm{J} 104439.4+764834$ & 2113 & 27 & 3 & 9.40 & 60 & 10.49 & 27.3 & & 1.12 \\
\hline & UGC05841 & $\mathrm{J} 104456$ & 2004 & 83 & 5. & 11.93 & & & & & \\
\hline 197 & NGC3348 & $\mathrm{J} 104710.0+725023$ & 2993 & 27 & -5 & 7.94 & 299 & 11.40 & 20.2 & 11.4 & 0.04 \\
\hline & NGC3364 & +722530 & 2874 & 41 & 5. & 10.10 & & & & & \\
\hline 198 & UGC05897 & $\mathrm{J} 104741.5+110437$ & 2575 & 16 & 5 & 10.25 & 116 & 10.42 & 22.5 & 21.0 & 0.42 \\
\hline & CGCG... & +105352 & 2640 & 5 & 6 & 11.72 & & & & & \\
\hline 199 & NGC3398 & J105131.4+552328 & 2943 & 75 & 3 & 10.76 & 388 & 10.25 & 100.8 & & 0.77 \\
\hline & MCG... & J104835. & 3006 & 37 & 9 & 14.02 & & & & & \\
\hline 200 & NGC3434 & $\mathrm{J} 105158.0+034731$ & 3445 & 9 & 3 & 9.74 & 37 & 10.77 & 1.4 & 1.3 & 0.09 \\
\hline & CGCG... & $\mathrm{J} 105200.2+035010$ & 3402 & 5 & 8 & 13.61 & & & & & \\
\hline 201 & NGC3432 & $\mathrm{J} 105231.1+363708$ & 589 & 5 & 9 & 9.06 & 158 & 9.52 & 3.1 & & 0.70 \\
\hline & CGCG... & J105747.0+361539 & 596 & 17 & 10 & 13.32 & & & & & \\
\hline 202 & CGCG... & $\mathrm{J} 105248.6+000204$ & 1607 & 5 & 9 & 12.97 & 25 & 8.82 & 65.3 & & 0.91 \\
\hline & MGC0013223 & J105240.6-000117 & 1569 & 75 & 10 & 16.40 & & & & & \\
\hline 203 & LSBC... & $\mathrm{J} 105318.6+023734$ & 851 & 10 & 9 & 13.45 & 30 & 8.17 & 18.9 & & 0.50 \\
\hline & LSBC... & J105303.1+022937 & 860 & 5 & 10 & 14.89 & & & & & \\
\hline 204 & NGC3448 & J105439.2+541819 & 1448 & 27 & 3 & 9.47 & 24 & 10.16 & 26.5 & 25.7 & 0.95 \\
\hline & UGC06016 & J105412.8+541714 & 1564 & 10 & 10 & 14.60 & & & & & \\
\hline 205 & ESO376-027 & J105658.2-330952 & 3436 & 53 & 5 & 11.84 & 4 & 10.08 & 6.1 & 3.0 & 0.97 \\
\hline & ESO-LV ... & J105659.1-330939 & 3560 & 40 & 7 & 12.92 & & & & & \\
\hline 206 & ESO569-030 & J105824.2-190912 & 3257 & 30 & 4 . & 13.06 & 62 & 9.57 & 64.5 & & 0.08 \\
\hline & $2 \mathrm{MASX} \ldots$ & J105844.2-190931 & 3314 & 74 & 4. & 13.82 & & & & & \\
\hline 207 & UGC06074 & $\mathrm{J} 105958.3+505411$ & 2946 & 5 & 9 & 10.57 & 41 & 10.31 & 0.1 & & 1.41 \\
\hline & MCG... & $\mathrm{J} 110006.4+505056$ & 2952 & 5 & 8 & 14.03 & & & & & \\
\hline 208 & BTS029 & J110138.9+303629 & 1626 & 5 & 10 & 14.08 & 12 & 8.61 & 240.3 & & 0.80 \\
\hline
\end{tabular}


Table I: Table. (Contd.)

\begin{tabular}{|c|c|c|c|c|c|c|c|c|c|c|c|}
\hline No. & Name & J2000.0 & \begin{tabular}{|l|}
$V_{L G}$ \\
$\mathrm{~km} \mathrm{~s}$ \\
\end{tabular} & & $\mathrm{~T}$ & $\begin{array}{r}K \\
\mathrm{mag}\end{array}$ & $\begin{array}{l}R_{\perp} \\
\mathrm{kpc}\end{array}$ & $\begin{array}{r}\log L \\
L_{\odot}\end{array}$ & $\begin{array}{r}\frac{M}{L_{K}} \\
\odot\end{array}$ & $\begin{array}{r}\frac{M^{c}}{L_{K}} \\
\odot\end{array}$ & $\log (I I)$ \\
\hline & BTS028 & J110132.4+303516 & 1708 & 75 & 9 & 14.58 & \multirow{3}{*}{67} & \multirow{3}{*}{10.67} & \multirow{3}{*}{42.7} & \multirow{3}{*}{42.4} & \multirow{3}{*}{0.15} \\
\hline \multirow[t]{2}{*}{209} & NGC3504 & $\mathrm{J} 110311.2+275821$ & 1467 & 8 & 2 & 8.26 & & & & & \\
\hline & NGC3512 & $\mathrm{J} 110403.0+280213$ & 1307 & 7 & 5 & 9.64 & & & & & \\
\hline \multirow[t]{2}{*}{210} & NGC3547 & J110955.9+104315 & 1428 & 5 & 3 & 10.43 & \multirow[t]{2}{*}{59} & \multirow[t]{2}{*}{9.76} & \multirow[t]{2}{*}{20.2} & & \multirow[t]{2}{*}{0.36} \\
\hline & CGCG.. & J110923.3+105003 & 1387 & 75 & 9 & 12.64 & & & & & \\
\hline \multirow[t]{2}{*}{211} & NGC3543 & J111056.4+612049 & 1779 & 5 & 4 & 11.40 & \multirow[t]{2}{*}{200} & \multirow[t]{2}{*}{9.56} & \multirow[t]{2}{*}{1.2} & & \multirow[t]{2}{*}{0.57} \\
\hline & {$[\mathrm{HS} 98] 137 \mathrm{~A}$} & $\mathrm{~J} 110718.0+613127$ & 1783 & 5 & 10 & 14.20 & & & & & \\
\hline \multirow[t]{2}{*}{212} & NGC3549 & J111056.9+532316 & 2932 & 5 & 5 & 9.18 & \multirow[t]{2}{*}{370} & \multirow[t]{2}{*}{10.86} & \multirow[t]{2}{*}{0.0} & & \multirow[t]{2}{*}{0.64} \\
\hline & CGCG... & $\mathrm{J} 111350.5+530511$ & 2934 & 40 & 7 & 13.13 & & & & & \\
\hline \multirow[t]{2}{*}{213} & NGC3544 & J111130.5-181722 & 3465 & 20 & 1 & 8.97 & \multirow[t]{2}{*}{66} & \multirow[t]{2}{*}{11.07} & 5.1 & 2.7 & 0.24 \\
\hline & NGC3571:... & J111119.6-181315 & 3378 & 30 & 8 & 14.15 & & & & & \\
\hline 214 & ESO377-019 & J111045.4-352102 & 2699 & 11 & 7 & 11.11 & 327 & 10.23 & 121.8 & & 0.13 \\
\hline & $2 \mathrm{MASX} \ldots$ & $\mathrm{J} 111252.5-353614$ & 2772 & 74 & 6 & 11.58 & & & & & \\
\hline 215 & CGCG... & $\mathrm{J} 111540.4+460739$ & 2901 & 5 & 9 & 13.31 & 23 & 9.25 & 17.7 & 16.1 & 0.75 \\
\hline & SDSS... & $\mathrm{J} 111550.5+460638$ & 2934 & 5 & 9 & 15.41 & & & & & \\
\hline 216 & UGC06309 & $\mathrm{J} 111746.5+512836$ & 2931 & 6 & 4 & 10.57 & 40 & 10.30 & 12.3 & 12.0 & 0.39 \\
\hline & MRK1445 & $\mathrm{J} 111732.3+512553$ & 2860 & 5 & 9 & 14.23 & & & & & \\
\hline 217 & NGC3617 & J111750.9-260804 & 1886 & 8 & -4 & 9.76 & 43 & 10.25 & 38.4 & 38.1 & 0.78 \\
\hline & ESO503-011 & J111744.4-260223 & 1770 & 5 & 6 & 12.08 & & & & & \\
\hline 218 & NGC3621 & J111816.5-324851 & 437 & 5 & 7 & 6.57 & 317 & 10.21 & 18.2 & 10.8 & 0.80 \\
\hline & HIPASS... & J113311.1-325745 & 409 & 9 & 9 & 13.65 & & & & & \\
\hline 219 & NGC3614 & $\mathrm{J} 111821.3+454454$ & 2366 & 14 & 5 & 9.63 & 34 & 10.47 & 4.5 & & 0.36 \\
\hline & SDSS... & $\mathrm{J} 111826.7+454124$ & 2308 & 35 & 9 & 15.47 & & & & & \\
\hline 220 & UGC06355 & $\mathrm{J} 112039.8+311320$ & 2142 & 5 & 6 & 12.52 & 23 & 9.38 & 13.8 & & 1.46 \\
\hline & $2 \mathrm{MASX} .$. & $\mathrm{J} 112046.0+311059$ & 2107 & 30 & 9 & 13.44 & & & & & \\
\hline 221 & NGC3631 & $\mathrm{J} 112102.9+531011$ & 1227 & 7 & 5 & 7.98 & 150 & 10.64 & 12.9 & 11.7 & 0.13 \\
\hline & NGC3657 & $\mathrm{J} 112355.6+525515$ & 1283 & 9 & -1 & 10.28 & & & & & \\
\hline 222 & UGC06402 & J112319.1-005521 & 2412 & 5 & 8 & 11.21 & 80 & 9.89 & 14.7 & 4.2 & 1.70 \\
\hline & SDSS... & J112344.8-005011 & 2446 & 15 & 10 & 14.49 & & & & & \\
\hline 223 & NGC3687 & J112800.6+293039 & 2453 & 12 & 4 & 10.23 & 7 & 10.23 & 40.5 & 39.9 & 1.46 \\
\hline & $2 \mathrm{MASX} \ldots$ & $\mathrm{J} 112804.2+293038$ & 2168 & 18 & 9 & 14.38 & & & & & \\
\hline 224 & NGC3694 & $\mathrm{J} 112854.1+352450$ & 2254 & 27 & 1 & 10.39 & 280 & 10.14 & 60.8 & 57.0 & 0.86 \\
\hline & UGC06499 & $\mathrm{J} 113011.3+355208$ & 2204 & 5 & 10 & 14.56 & & & & & \\
\hline 225 & NGC3705 & $\mathrm{J} 113007.5+091636$ & 868 & 5 & 2 & 7.90 & 190 & 10.31 & 2.6 & & 0.14 \\
\hline & IC2828 & $\mathrm{J} 112710.9+084352$ & 883 & 12 & 9 & 12.64 & & & & & \\
\hline 226 & SBS1129+576 & $\mathrm{J} 113202.5+572246$ & 1660 & 5 & 8 & 14.48 & 23 & 8.44 & 19.9 & & 0.09 \\
\hline & SDSS... & $\mathrm{J} 113227.7+572142$ & 1646 & 33 & 9 & 15.02 & & & & & \\
\hline 227 & IC0705 & $\mathrm{J} 113256.3+501430$ & 3005 & 42 & -1 & 11.41 & 175 & 10.01 & 65.6 & 37.7 & 0.00 \\
\hline
\end{tabular}


Table I: Table. (Contd.)

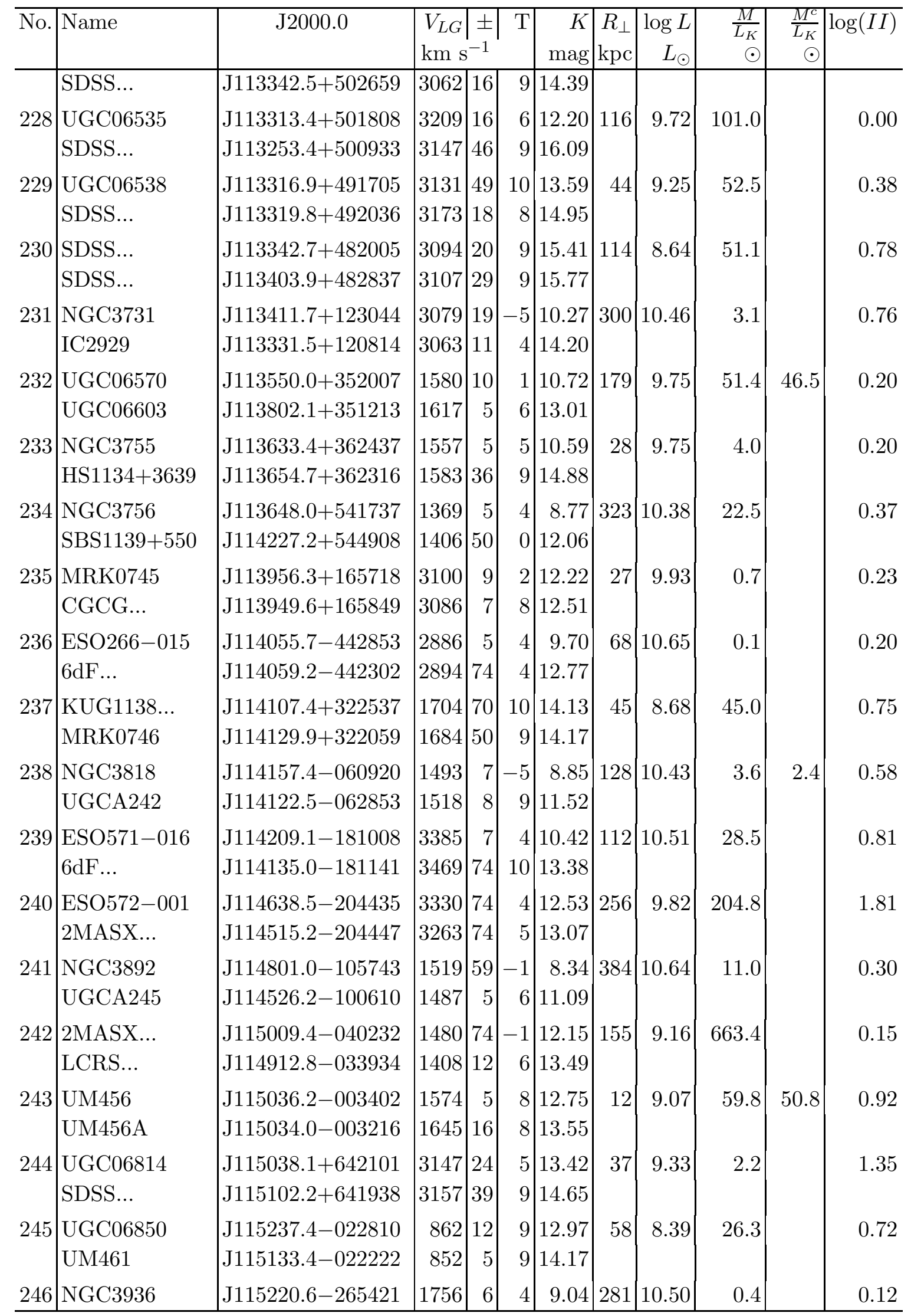


Table I: Table. (Contd.)

\begin{tabular}{|c|c|c|c|c|c|c|c|c|c|c|c|}
\hline \multirow[t]{2}{*}{ No. } & Name & $\mathrm{J} 2000.0$ & $\begin{array}{l}V_{L G} \\
\mathrm{~km} \mathrm{~s}\end{array}$ & \begin{tabular}{|c|} 
\pm \\
-1
\end{tabular} & $\mathrm{~T}$ & $\begin{array}{r}K \\
\mathrm{mag}\end{array}$ & $\begin{array}{l}R_{\perp} \\
\mathrm{kpc}\end{array}$ & $\begin{array}{r}\log L \\
L_{\odot}\end{array}$ & $\begin{array}{r}\frac{M}{L_{K}} \\
\odot\end{array}$ & $\begin{array}{r}\frac{M^{c}}{L_{K}} \\
\odot\end{array}$ & $\log (I I)$ \\
\hline & ESO504-028 & $115454.4-271505$ & 1762 & 5 & 7 . & 11.41 & & & & & \\
\hline \multirow[t]{2}{*}{247} & NGC3952 & 15340.6 & 1382 & 5 & 9 & 11.00 & 103 & 9.74 & 2.5 & & 0.35 \\
\hline & IC2969 & J115231.3 & 1372 & 6 & 4. & 11.14 & & & & & \\
\hline \multirow[t]{2}{*}{248} & KDG083 & J115614.5+311816 & 617 & 5 & -3 & 11.31 & 125 & 8.72 & 165.0 & & 0.43 \\
\hline & KUG1157... & $\mathrm{J} 120016.2+311330$ & 593 & 28 & 4. & 12.87 & & & & & \\
\hline \multirow[t]{2}{*}{249} & UGC06927 & $\mathrm{J} 115708.5$ & 3334 & 32 & -2 & 10.61 & 443 & 10.43 & 38.7 & 24.0 & 0.46 \\
\hline & IC2985 & J115912 & 3290 & 10 & 7 . & 13.06 & & & & & \\
\hline \multirow[t]{2}{*}{250} & IC2986 & J115949.6+305040 & 3107 & 21 & -2 & 11.17 & 195 & 10.14 & 27.8 & & 0.21 \\
\hline & CGCG. & J120103. & 3066 & 40 & 5. & 13.49 & & & & & \\
\hline \multirow[t]{2}{*}{251} & IC0755 & $\mathrm{J} 120110.4$ & 1396 & 8 & 3 . & 11.32 & 33 & 9.47 & 11.4 & 8.5 & 0.25 \\
\hline & MRK0756 & J120127 & 1366 & 8 & 4 . & 12.39 & & & & & \\
\hline \multirow[t]{2}{*}{252} & KUG1159. & J120225. & 3336 & 32 & 7 . & 12.96 & 113 & 9.48 & 40.7 & & 0.73 \\
\hline & KISSB083 & J120303 & 3366 & 75 & 9 & 16.03 & & & & & \\
\hline \multirow[t]{2}{*}{253} & SBS1203+592 & $\mathrm{J} 120614.2+585816$ & 3367 & 7 & 9 . & 13.78 & 62 & 9.23 & 0.2 & & 0.39 \\
\hline & SDSS... & J12054 & 3365 & 62 & 8 & 15.16 & & & & & \\
\hline \multirow[t]{2}{*}{254} & NGC4112 & J120709.5-401229 & 2400 & 45 & 3 & 9.17 & 23 & 10.69 & 2.9 & & 0.02 \\
\hline & ESO321-007 & J120722.1-401304 & 2329 & 74 & 5. & 12.49 & & & & & \\
\hline \multirow[t]{2}{*}{255} & NGC4119 & J120809.6+102244 & 1525 & 26 & -1 & 8.48 & 343 & 10.55 & 9.3 & 8.1 & 0.30 \\
\hline & SDSS... & J121201. & 1496 & 5 & 8 & 15.08 & & & & & \\
\hline \multirow[t]{2}{*}{256} & NGC4127 & $\mathrm{J} 120826.4+764815$ & 2004 & 5 & 5. & 10.01 & 333 & 10.21 & 33.0 & 20.8 & 0.36 \\
\hline & UGC07265 & J121504. & 2041 & 11 & 8 & 13.79 & & & & & \\
\hline \multirow[t]{2}{*}{257} & ESO441-012 & J120927.2-323055 & 1913 & 10 & 2 & 10.17 & 262 & 10.15 & 40.4 & 36.5 & 0.00 \\
\hline & ESO441-007 & J120815.6-320031 & 1956 & 6 & 4 & 12.30 & & & & & \\
\hline \multirow[t]{2}{*}{258} & NGC4141 & J120947.3+585057 & 2079 & 57 & 6 . & 11.36 & 59 & 9.70 & 5.4 & & 1.10 \\
\hline & SDSS... & $\mathrm{J} 120858.7+584732$ & 2060 & 16 & 8. & 14.59 & & & & & \\
\hline \multirow[t]{2}{*}{259} & ESO321-010 & J121142.1-383255 & 2861 & 6 & 4 & 10.34 & 410 & 10.39 & 7.7 & & 0.12 \\
\hline & $6 \mathrm{dF} \ldots$ & J121143.0-390845 & 2881 & 74 & 9 & 13.59 & & & & & \\
\hline \multirow[t]{2}{*}{260} & NGC4162 & $\mathrm{J} 121152.5+240725$ & 2512 & 5 & 4 & 9.35 & 69 & 10.64 & 5.4 & & 0.07 \\
\hline & KUG1209... & J121134.9+240144 & 2458 & 75 & 10 & 14.63 & & & & & \\
\hline \multirow[t]{2}{*}{261} & NGC4165 & $\mathrm{J} 121211.8+131448$ & 1764 & 17 & 2 & 10.48 & 120 & 9.91 & 28.5 & 26.1 & 0.29 \\
\hline & SDSS... & J121317.8+130936 & 1804 & 5 & 8. & 14.08 & & & & & \\
\hline \multirow[t]{2}{*}{262} & NGC4194 & $\mathrm{J} 121409.5+543137$ & 2603 & 18 & 9 & 9.62 & 382 & 10.58 & 10.3 & 9.1 & 0.45 \\
\hline & SDSS... & $\mathrm{J} 121250.4+550625$ & 2633 & 5 & 9 & 15.38 & & & & & \\
\hline \multirow[t]{2}{*}{263} & UGC07249 & $\mathrm{J} 121436.9+124843$ & 509 & 5 & 8 . & 12.73 & 13 & 7.96 & 11.6 & & 0.27 \\
\hline & SDSS... & $\mathrm{J} 121412.1+124658$ & 501 & 11 & 8 & 14.89 & & & & & \\
\hline \multirow[t]{2}{*}{264} & NGC4203 & $\mathrm{J} 121505.1+331150$ & 1078 & 5 & -3 & 7.40 & 483 & 10.71 & 24.5 & 23.4 & 0.13 \\
\hline & UGC07428 & $\mathrm{J} 122202.5+320543$ & 1125 & 5 & 10 & 12.83 & & & & & \\
\hline 265 & ESO321-016 & J121526.7-380839 & 2901 & 8 & 6 . & 10.89 & 72 & 10.20 & 1.3 & 0.7 & 0.12 \\
\hline
\end{tabular}


Table I: Table. (Contd.)

\begin{tabular}{|c|c|c|c|c|c|c|c|c|c|c|c|}
\hline \multirow[t]{2}{*}{ No. } & Name & J2000.0 & \begin{tabular}{|l|}
$V_{L G}$ \\
$\mathrm{~km} \mathrm{~s}$
\end{tabular} & \begin{tabular}{|l|} 
\pm \\
-1
\end{tabular} & $\mathrm{~T}$ & \multirow{2}{*}{\begin{tabular}{|r}
$\begin{array}{r}K \\
\mathrm{mag}\end{array}$ \\
13.30
\end{tabular}} & \multirow[t]{2}{*}{$\begin{array}{l}R_{\perp} \\
\mathrm{kpc}\end{array}$} & \multirow[t]{2}{*}{$\begin{array}{r}\log L \\
L_{\odot} \\
\end{array}$} & \multirow[t]{2}{*}{$\begin{array}{r}\frac{M}{L_{K}} \\
\odot \\
\end{array}$} & \multirow[t]{2}{*}{$\begin{array}{r}\frac{M^{c}}{L_{K}} \\
\odot \\
\end{array}$} & \multirow[t]{2}{*}{$\log (I I)$} \\
\hline & ESO321-018 & $121554.3-380536$ & 2885 & 5 & 10 & & & & & & \\
\hline \multirow[t]{2}{*}{266} & NGC4217 & 121550.9 & 1085 & 5 & 3 & 7.58 & \multirow[t]{2}{*}{199} & \multirow[t]{2}{*}{10.80} & \multirow[t]{2}{*}{18.0} & \multirow[t]{2}{*}{6.4} & \multirow[t]{2}{*}{0.20} \\
\hline & NGC4220 & $\mathrm{J} 121611.7+475260$ & 1015 & 35 & 0 & 8.13 & & & & & \\
\hline \multirow[t]{2}{*}{267} & UGC07298 & J121630.1+521339 & 254 & 5 & 10 & 12.55 & \multirow[t]{2}{*}{12} & \multirow[t]{2}{*}{7.44} & \multirow[t]{2}{*}{44.8} & & \multirow[t]{2}{*}{0.70} \\
\hline & CGCG... & $\mathrm{J} 121546.8+522317$ & 245 & 15 & 10 & 14.25 & & & & & \\
\hline \multirow[t]{2}{*}{268} & NGC4238 & $\mathrm{J} 121655.8+632436$ & 2908 & 11 & 7 . & 11.03 & \multirow[t]{2}{*}{52} & \multirow[t]{2}{*}{10.11} & 2.4 & 0.1 & 0.64 \\
\hline & SDSS... & $\mathrm{J} 121617.2+63$ & 2930 & 11 & 9 & 15.46 & & & & & \\
\hline 269 & ESO380-008 & $\mathrm{J} 121658.2-372816$ & 1835 & 5 & 8. & 11.40 & 79 & 9.59 & 1.4 & & 1.16 \\
\hline & ESO380-009 & $\mathrm{J} 121731.9$ & 1828 & 9 & 10 & 14.06 & & & & & \\
\hline 270 & NGC4290 & $\mathrm{J} 122047.5+580533$ & 3143 & 9 & 3 & 9.29 & 610 & 10.88 & 0.6 & & 0.37 \\
\hline & MCG... & $\mathrm{J} 122634.9$ & 3151 & 67 & 6 & 13.22 & & & & & \\
\hline 271 & KUG1218. & $\mathrm{J} 122054.9$ & 623 & 46 & 9 . & 12.97 & 68 & 8.02 & 1287.8 & 395.8 & 0.29 \\
\hline & KDG & J12214 & 582 & 5 & 10 & 15.07 & & & & & \\
\hline 272 & VCC0513 & $\mathrm{J} 122157.8+02$ & 1699 & 5 & 2 & 12.07 & 191 & 9.43 & 0.3 & & 0.06 \\
\hline & VCC0597 & $\mathrm{J} 122256$. & 1697 & 16 & 3 & 12.56 & & & & & \\
\hline 273 & NGC4369 & $\mathrm{J} 122436.2+392259$ & 1053 & 29 & 1 & 8.91 & 154 & 10.09 & 29.7 & 28.0 & 0.03 \\
\hline & PGC166134 & $\mathrm{J} 122207.0+3$ & 1098 & 5 & 10 & 14.66 & & & & & \\
\hline 274 & SBS1222+614 & $\mathrm{J} 122505.4+61$ & 832 & 5 & 9 & 13.03 & 18 & 8.48 & 0.1 & & 0.86 \\
\hline & MCG... & $\mathrm{J} 122453$. & 833 & 5 & 10 & 13.20 & & & & & \\
\hline 275 & NGC4384 & $\mathrm{J} 122512.0+54$ & 2616 & 11 & 1 . & 10.37 & 155 & 10.27 & 13.9 & 12.9 & 0.66 \\
\hline & SDSS... & $\mathrm{J} 122442 . \mathrm{C}$ & 2578 & 5 & 10 & 14.99 & & & & & \\
\hline 276 & CGCG.. & $\mathrm{J} 122536.4+502013$ & 2563 & 16 & 4 & 12.12 & 14 & 9.61 & 5.3 & 2.8 & 2.11 \\
\hline & 2MASX... & $\mathrm{J} 122528.4$ & 2599 & 12 & 6 & 14.45 & & & & & \\
\hline 277 & UGC07531 & J122612.0-011813 & 1836 & 42 & 10 & 12.74 & 29 & 9.11 & 133.5 & & 0.71 \\
\hline & UM501 & $\mathrm{J} 122622.7$ & 1765 & 60 & 9 . & 14.04 & & & & & \\
\hline 278 & NGC4433 & J122738.6-081642 & 2794 & 10 & 2 & 9.50 & 79 & 10.95 & 0.0 & & 1.42 \\
\hline & NGC4428 & J122728.3-081004 & 2801 & 11 & 5 & 9.64 & & & & & \\
\hline 279 & ESO380-033 & $\mathrm{J} 122744.3-342520$ & 2659 & 9 & 4. & 11.91 & 46 & 9.84 & 36.5 & & 0.37 \\
\hline & 2MASX.. & J122724.9-342335 & 2590 & 74 & 9 & 12.67 & & & & & \\
\hline 280 & UGC07584 & $\mathrm{J} 122802.8+223516$ & 543 & 5 & 9 . & 13.75 & 39 & 7.72 & 0.0 & & 0.40 \\
\hline & LSBC... & $\mathrm{J} 122805.0+221727$ & 543 & 5 & 10 & 14.56 & & & & & \\
\hline 281 & NGC4449 & $\mathrm{J} 122811.9+440540$ & 252 & 7 & 9 & 7.24 & 36 & 9.49 & 2.0 & 0.6 & 0.37 \\
\hline & UGC07577 & J122740.9+432944 & 240 & 5 & 10 & 10.44 & & & & & \\
\hline 282 & NGC4454 & J122850.8-015621 & 2206 & 44 & 0 & 9.04 & 65 & 10.70 & 3.5 & 1.6 & 0.23 \\
\hline & CGCG... & J122850.7-020339 & 2254 & 13 & 4. & 11.71 & & & & & \\
\hline 283 & NGC4513 & $\mathrm{J} 123201.5+661957$ & 2451 & 26 & 1 . & 10.21 & 275 & 10.30 & 32.9 & & 0.87 \\
\hline & SDSS... & $\mathrm{J} 123602.3+660618$ & 2497 & 58 & 6 . & 13.73 & & & & & \\
\hline 284 & ESO322-031 & J123648.7-420827 & 3236 & 59 & -1 & 9.75 & 659 & 10.98 & 28.1 & & 0.11 \\
\hline
\end{tabular}


Table I: Table. (Contd.)

\begin{tabular}{|c|c|c|c|c|c|c|c|c|c|c|c|}
\hline No & Name & J2000.0 & $\begin{array}{l}\mid V_{L G} \\
\mathrm{~km} \mathrm{~s}\end{array}$ & & & $\begin{array}{r}K \\
\mathrm{mag}\end{array}$ & \begin{tabular}{l|}
$R_{\perp}$ \\
$\mathrm{kpc}$
\end{tabular} & $\left|\begin{array}{r}\log L \\
L_{\odot}\end{array}\right|$ & $\begin{array}{r}\frac{M}{L_{K}} \\
\odot\end{array}$ & $\begin{array}{r}\frac{M^{c}}{L_{K}} \\
\odot\end{array}$ & $\log (I I)$ \\
\hline & ESO322-034 & J123733.3-411829 & 3295 & $59-$ & $\overline{-2}$ & 9.91 & & & & & \\
\hline 285 & \begin{tabular}{|l} 
NGC4584 \\
IC3586
\end{tabular} & $\begin{array}{l}\mathrm{J} 123817.9+130635 \\
\mathrm{~J} 123654.8+123112\end{array}$ & $\left|\begin{array}{l}1641 \\
1645\end{array}\right|$ & $\begin{array}{r}18 \\
5\end{array}$ & & $\begin{array}{l}10.45 \\
12.11\end{array}$ & 267 & 9.92 & 0.6 & & 0.11 \\
\hline 286 & $\begin{array}{l}\text { UGC07798 } \\
\text { SDSS... }\end{array}$ & \begin{tabular}{|l}
$\mathrm{J} 123803.2-021551$ \\
$\mathrm{~J} 123905.8-020044$
\end{tabular} & $\left|\begin{array}{l}2418 \\
2446\end{array}\right|$ & $\begin{array}{r}17 \\
5\end{array}$ & & $\begin{array}{l}11.59 \\
14.29\end{array}$ & 211 & 9.75 & 35.3 & 27.6 & 0.74 \\
\hline 287 & $\begin{array}{l}\text { NGC4628 } \\
\text { NGC4626 }\end{array}$ & $\begin{array}{l}\mathrm{J} 124225.3-065816 \\
\mathrm{~J} 124225.1-070245\end{array}$ & & $\begin{array}{r}7 \\
34\end{array}$ & $\begin{array}{l}3 \\
4\end{array} \mid$ & $\begin{array}{r}9.45 \\
10.94\end{array}$ & 48 & 10.76 & 4.5 & 0.8 & 0.00 \\
\hline 288 & $\begin{array}{l}\text { NGC4630 } \\
\text { VCC1855 }\end{array}$ & $\begin{array}{l}\mathrm{J} 124231.2+035737 \\
\mathrm{~J} 124050.3+043133\end{array}$ & $\begin{array}{l}556 \\
586\end{array}$ & $\begin{array}{l}36 \\
40\end{array}$ & \begin{tabular}{r|r}
10 \\
1
\end{tabular} \mid & $\begin{array}{r}9.88 \\
14.48\end{array}$ & 96 & 9.15 & 68.3 & & 0.30 \\
\hline 289 & $\begin{array}{l}\text { UGC07883 } \\
\text { SDSS... }\end{array}$ & \begin{tabular}{|l}
$\mathrm{J} 124257.3-011346$ \\
$\mathrm{~J} 124309.6-011234$
\end{tabular} & \begin{tabular}{|l|}
3061 \\
2923
\end{tabular} & $\begin{array}{l}18 \\
13\end{array}$ & & $\begin{array}{l}10.57 \\
15.69\end{array}$ & 39 & 10.31 & 43.6 & 42.0 & 1.02 \\
\hline 290 & $\begin{array}{l}\text { NGC4653 } \\
\text { NGC4642 }\end{array}$ & \begin{tabular}{|l}
$\mathrm{J} 124350.9-003340$ \\
$\mathrm{~J} 124317.8-003839$
\end{tabular} & \begin{tabular}{|l|}
2476 \\
2494
\end{tabular} & $\begin{array}{l}6 \\
5\end{array}$ & & $\begin{array}{r}9.96 \\
10.36\end{array}$ & 96 & 10.62 & 0.8 & 0.5 & 0.74 \\
\hline 291 & $\begin{array}{l}\text { SDSS... } \\
\text { SDSS... }\end{array}$ & $\begin{array}{l}\mathrm{J} 124423.2+620306 \\
\mathrm{~J} 124412.1+621019\end{array}$ & & $\begin{array}{r}72 \\
8\end{array}$ & \begin{tabular}{l|l}
10 & 1 \\
10 & 1
\end{tabular} & $\begin{array}{c}15.20 \\
15.40\end{array}$ & 78 & 8.62 & 102.0 & & 0.94 \\
\hline 292 & $\begin{array}{l}\text { ESO268-044 } \\
\text { 2MASX... }\end{array}$ & $\begin{array}{l}\mathrm{J} 124842.2-450029 \\
\mathrm{~J} 124805.2-451909\end{array}$ & $\left|\begin{array}{l}3233 \\
3181\end{array}\right|$ & $\begin{array}{c}30 \\
74\end{array}$ & \begin{tabular}{r|r}
3 & 1 \\
-1 & 1
\end{tabular} & $\begin{array}{c}10.59 \\
11.84\end{array}$ & 253 & 10.48 & 26.3 & & 0.42 \\
\hline 293 & $\begin{array}{l}\text { ESO575-012 } \\
\text { ESO575-003 }\end{array}$ & \begin{tabular}{|l}
$\mathrm{J} 125147.4-221658$ \\
$\mathrm{~J} 125031.7-221205$
\end{tabular} & \begin{tabular}{|}
3367 \\
3471
\end{tabular} & $\begin{array}{l}74 \\
74\end{array}$ & & $\begin{array}{l}12.47 \\
13.75\end{array}$ & 248 & 9.78 & 520.5 & & 0.37 \\
\hline 294 & $\begin{array}{l}\text { ESO575-017 } \\
\text { ESO575-019 }\end{array}$ & $\begin{array}{l}\mathrm{J} 125240.2-221134 \\
\mathrm{~J} 125327.9-222053\end{array}$ & & $\begin{array}{r}29 \\
5\end{array}$ & & $\begin{array}{l}11.60 \\
12.75\end{array}$ & 186 & $10.09 \mid$ & 3.5 & & 0.12 \\
\hline 295 & $\begin{array}{l}\text { ESO507-036 } \\
\text { AM1251-240. }\end{array}$ & \begin{tabular}{|l}
$\mathrm{J} 125259.2-240326$ \\
$\mathrm{~J} 125406.5-242538$
\end{tabular} & \begin{tabular}{|l|}
3138 \\
3077
\end{tabular} & $\begin{array}{l}10 \\
74\end{array}$ & & $\begin{array}{l}11.22 \\
14.03\end{array}$ & 334 & 10.11 & 114.2 & & 0.25 \\
\hline 296 & $\begin{array}{l}\text { NGC4793 } \\
\text { KISSR0148 }\end{array}$ & $\begin{array}{l}\mathrm{J} 125440.7+285618 \\
\mathrm{~J} 125445.2+285529\end{array}$ & $\mid \begin{array}{l}2474 \\
2336\end{array}$ & $\begin{array}{l}20 \\
67\end{array}$ & \begin{tabular}{r|r}
5 \\
10
\end{tabular} & $\begin{array}{r}8.48 \\
15.60\end{array}$ & 12 & 10.96 & 3.0 & 0.2 & 1.00 \\
\hline 297 & $\begin{array}{l}\text { NGC4767B } \\
\text { ESO323-040 }\end{array}$ & \begin{tabular}{|l}
$\mathrm{J} 125445.0-395108$ \\
$\mathrm{~J} 125428.5-394341$
\end{tabular} & \begin{tabular}{|c|}
3241 \\
3325
\end{tabular} & $\begin{array}{l}18 \\
74\end{array}$ & \begin{tabular}{l|l}
6 \\
4 \\
1
\end{tabular} & $\begin{array}{r}9.79 \\
12.07\end{array}$ & 106 & 10.75 & 15.7 & & 0.21 \\
\hline 298 & $\begin{array}{l}\text { 2MASX... } \\
\text { ESO443-010 }\end{array}$ & \begin{tabular}{|l}
$\mathrm{J} 125557.3-283846$ \\
$\mathrm{~J} 125532.6-282733$
\end{tabular} & $\begin{array}{l}2929 \\
2950\end{array}$ & $\begin{array}{r}35 \\
7\end{array}$ & \begin{tabular}{l|l}
0 & 1 \\
2 & 1
\end{tabular} & 11.19 & 146 & 10.32 & 3.4 & & 0.09 \\
\hline 299 & $\begin{array}{l}\text { ARK396 } \\
\text { SDSS... }\end{array}$ & $\begin{array}{l}\mathrm{J} 125726.5-032927 \\
\mathrm{~J} 125722.9-034028\end{array}$ & $\mid \begin{array}{l}2947 \\
3079\end{array}$ & & & $\begin{array}{l}10.34 \\
14.96\end{array}$ & 133 & 10.41 & 105.8 & & 0.42 \\
\hline 300 & $\begin{array}{l}\text { NGC4830 } \\
\text { 2MASX... }\end{array}$ & \begin{tabular}{|l} 
J125727.9-194129 \\
J125732.7-194201
\end{tabular} & \begin{tabular}{|c|}
3142 \\
3498
\end{tabular} & $\begin{array}{l}12 \\
74\end{array}$ & \begin{tabular}{l|l}
-3 \\
-1
\end{tabular} \mid & $\begin{array}{r}8.82 \\
12.46\end{array}$ & 17 & 11.12 & 19.0 & 15.8 & 0.82 \\
\hline 301 & $\begin{array}{l}\text { UGC08127 } \\
\text { UGC08127... }\end{array}$ & \begin{tabular}{|l}
$\mathrm{J} 130103.7-015712$ \\
$\mathrm{~J} 130100.7-015834$
\end{tabular} & \begin{tabular}{|l|}
1297 \\
1302
\end{tabular} & $\begin{array}{r}33 \\
5\end{array}$ & \begin{tabular}{l|l}
10 & 1 \\
10 & 1
\end{tabular} & $\begin{array}{c}12.68 \\
13.64\end{array}$ & 8 & 8.89 & 0.2 & & 0.07 \\
\hline 302 & $\begin{array}{l}\text { ESO323-068 } \\
\text { AM1300-412 }\end{array}$ & \begin{tabular}{|l} 
J130157.1-410414 \\
J130300.4-414215
\end{tabular} & \begin{tabular}{|c|}
3103 \\
3165
\end{tabular} & $\begin{array}{l}59 \\
74\end{array}$ & \begin{tabular}{l|l}
3 & 1 \\
3 & 1
\end{tabular} & $\begin{array}{c}10.32 \\
11.74\end{array}$ & 497 & 10.55 & 63.6 & & 0.09 \\
\hline 303 & UGCA319 & J130214.4-171415 & 548 & 8 & 101 & 11.59 & 40 & 8.53 & 0.8 & & 0.84 \\
\hline
\end{tabular}


Table I: Table. (Contd.)

\begin{tabular}{|c|c|c|c|c|c|c|c|c|c|c|c|}
\hline No. & Name & J2000.0 & \begin{tabular}{|c|}
$V_{L G}$ \\
$\mathrm{~km} \mathrm{~s}$ \\
\end{tabular} & & $\mathrm{~T}$ & $\begin{array}{r}K \\
\mathrm{mag}\end{array}$ & $\begin{array}{l}R_{\perp} \\
\mathrm{kpc}\end{array}$ & $\begin{array}{r}\log L \\
L_{\odot}\end{array}$ & $\begin{array}{r}\frac{M}{L_{K}} \\
\odot \\
\end{array}$ & $\begin{array}{r}\frac{M^{c}}{L_{K}} \\
\odot\end{array}$ & $\log (I I)$ \\
\hline & UGCA320 & J130316.7-172523 & 546 & 5 & 10 & 12.96 & & & & & \\
\hline \multirow[t]{2}{*}{304} & LCRS... & J130235.6-113807 & 3293 & 9 & 4 & 13.14 & 157 & 9.55 & 2.1 & & 0.33 \\
\hline & PGC045016 & J130231.4-112613 & 3287 & 88 & 9 & 13.84 & & & & & \\
\hline \multirow[t]{2}{*}{305} & UGC08153 & $\mathrm{J} 130305.9+035931$ & 2744 & 5 & 6 & 10.87 & 47 & 10.12 & 0.4 & & 1.65 \\
\hline & SDSS.. & $\mathrm{J} 130249.2+035836$ & 2753 & 55 & 10 & 15.73 & & & & & \\
\hline \multirow[t]{2}{*}{306} & NGC4961 & $\mathrm{J} 130547.6+274403$ & 2528 & 17 & 6 & 10.84 & 15 & 10.07 & 0.2 & 0.0 & 1.00 \\
\hline & LSBC.. & $\mathrm{J} 130549.7+274239$ & 2517 & 5 & 10 & 14.60 & & & & & \\
\hline \multirow[t]{2}{*}{307} & SBS1307+542 & $\mathrm{J} 130908.8+535637$ & 2608 & 33 & 3 & 12.13 & 94 & 9.83 & 11.6 & & 1.66 \\
\hline & UGC08231 & $\mathrm{J} 130837.6+540428$ & 2581 & 6 & 5 & 12.28 & & & & & \\
\hline \multirow[t]{2}{*}{308} & NGC4989 & J130916.0-052347 & 2888 & 38 & -2 & 9.23 & 88 & 10.97 & 22.0 & 20.1 & 0.12 \\
\hline & NGC4990 & J130917.3-051622 & 3029 & 7 & -2 & 10.35 & & & & & \\
\hline \multirow[t]{2}{*}{309} & NGC4995 & $\mathrm{J} 130940.7-075000$ & 1605 & 7 & 3 & 8.21 & 402 & 10.94 & 40.6 & 39.7 & 0.02 \\
\hline & NGC4981 & J130848.7-064639 & 1519 & 6 & 4 & 8.47 & & & & & \\
\hline \multirow[t]{2}{*}{310} & NGC4988 & J130954.4-430621 & 1842 & 42 & -1 & 11.85 & 48 & 9.42 & 20.8 & & 0.68 \\
\hline & ESO269-056 & J131001.6-431243 & 1873 & 15 & 10 & 14.49 & & & & & \\
\hline \multirow[t]{2}{*}{311} & UGC08246 & J131004.5+341051 & 833 & 5 & 7 & 13.14 & 21 & 8.26 & 214.2 & & 0.14 \\
\hline & MAPS-N... & $\mathrm{J} 131029.2+341413$ & 873 & 75 & 10 & 15.03 & & & & & \\
\hline \multirow[t]{2}{*}{312} & UGC08255 & $\mathrm{J} 131056.5+112838$ & 3285 & 6 & 6 & 11.38 & 184 & 10.16 & 27.7 & 26.0 & 0.20 \\
\hline & UGC08253 & $\mathrm{J} 131043.9+114228$ & 3242 & 5 & 6 & 12.90 & & & & & \\
\hline \multirow[t]{2}{*}{313} & ESO382-016 & J131312.4-364322 & 3032 & 33 & -2 & 9.36 & 164 & 10.92 & 34.2 & & 0.38 \\
\hline & $2 \mathrm{MASX} .$. & J131208.8-363934 & 3152 & 74 & 1 & 10.92 & & & & & \\
\hline \multirow[t]{2}{*}{314} & UGC08316 & $\mathrm{J} 131406.5+480923$ & 2619 & 34 & 4 & 12.49 & 151 & 9.45 & 94.2 & 69.9 & 1.13 \\
\hline & SDSS... & $\mathrm{J} 131239.0+480919$ & 2581 & 5 & 9 & 15.43 & & & & & \\
\hline \multirow[t]{2}{*}{315} & UGC08331 & $\mathrm{J} 131530.3+472956$ & 344 & 6 & 10 & 12.20 & 4 & 7.76 & 20.0 & & 0.20 \\
\hline & DDO169 NW & $\mathrm{J} 131518.4+473200$ & 328 & 75 & 10 & 16.61 & & & & & \\
\hline \multirow[t]{2}{*}{316} & IC4216 & J131701.9-104612 & 2664 & 8 & 6 & 10.73 & 407 & 10.36 & 13.3 & & 0.37 \\
\hline & NGC5066 & J131828.5-101402 & 2639 & 43 & 1 & 11.20 & & & & & \\
\hline \multirow[t]{2}{*}{317} & ESO576-024 & $\mathrm{J} 131757.5-215147$ & 2824 & 75 & 8 & 13.00 & 169 & 9.55 & 319.9 & & 0.65 \\
\hline & ESO576-023 & J131739.4-213711 & 2749 & 9 & 8 & 13.13 & & & & & \\
\hline \multirow[t]{2}{*}{318} & NGC5068 & J131854.8-210221 & 471 & 5 & 6 & 7.51 & 271 & 9.92 & 7.5 & & 0.65 \\
\hline & $2 \mathrm{MASX} \ldots$ & J132921.0-211045 & 457 & 29 & -2 & 12.56 & & & & & \\
\hline \multirow[t]{2}{*}{319} & ESO576-040 & J132043.7-220304 & 1885 & 12 & 7 & 11.37 & 10 & 9.63 & 0.1 & & 0.09 \\
\hline & [CCF97] G8 & J132049.6-220318 & 1879 & 6 & 5 & 13.66 & & & & & \\
\hline \multirow[t]{2}{*}{320} & NGC5144 & $\mathrm{J} 132254.1+703053$ & 3325 & 14 & 5 & 10.07 & 88 & $10.61 \mid$ & 9.7 & & 0.50 \\
\hline & UGC08434 & $\mathrm{J} 132413.9+703153$ & 3264 & 75 & 7 & 13.60 & & & & & \\
\hline \multirow[t]{2}{*}{321} & NGC5114 & J132401.7-322038 & 3357 & 26 & -3 & 9.27 & 180 & 11.07 & 43.5 & 34.8 & 0.20 \\
\hline & ESO 444-c & J132306.3-321441 & 3512 & 37 & -2 & 10.49 & & & & & \\
\hline 322 & NGC5122 & J132414.9-103915 & 2681 & 22 & -1 & 10.02 & 122 & 10.43 & 26.1 & 24.2 & 0.37 \\
\hline
\end{tabular}


Table I: Table. (Contd.)

\begin{tabular}{|c|c|c|c|c|c|c|c|c|c|c|c|}
\hline \multirow[t]{2}{*}{ No. } & Name & $\mathrm{J} 2000.0$ & $\begin{array}{l}V_{L G} \\
\mathrm{~km} \mathrm{~s}\end{array}$ & \begin{tabular}{|c|} 
\pm \\
-1
\end{tabular} & $\mathrm{~T}$ & $\begin{array}{r}K \\
\mathrm{mag}\end{array}$ & $\begin{array}{l}R_{\perp} \\
\mathrm{kpc}\end{array}$ & $\begin{array}{r}\log L \\
L_{\odot}\end{array}$ & $\begin{array}{r}\frac{M}{L_{K}} \\
\odot\end{array}$ & $\begin{array}{r}\frac{M^{c}}{L_{K}} \\
\odot\end{array}$ & $\log (I I)$ \\
\hline & MCG... & J132439.1-102921 & 2611 & 9 & 9 & 13.98 & & & & & \\
\hline \multirow[t]{2}{*}{323} & IC4237 & J132432.8-210813 & 2451 & 7 & 3 & 9.29 & 304 & 10.71 & 6.8 & & 0.67 \\
\hline & MCG... & J132516.0-203859 & 2482 & 29 & 4 & 11.52 & & & & & \\
\hline \multirow[t]{2}{*}{324} & UGC08521 & $\mathrm{J} 133230.8+015052$ & 3167 & 6 & 2 & 10.34 & 164 & 10.46 & 18.3 & & 0.81 \\
\hline & SDSS... & $\mathrm{J} 133140.6+014800$ & 3219 & 41 & 9 & 14.77 & & & & & \\
\hline \multirow[t]{2}{*}{325} & 2MASX. & J133549.8-341433 & 3086 & 30 & 4 & 12.82 & 201 & 9.68 & 234.0 & & 0.38 \\
\hline & $6 \mathrm{dF} \ldots$ & J133536.1-34 & 3017 & 74 & 5 & 13.06 & & & & & \\
\hline \multirow[t]{2}{*}{326} & UGC08602 & $\mathrm{J} 133645.5+320528$ & 3062 & 5 & 10 & 15.60 & 23 & 8.61 & 47.7 & 41.1 & 0.58 \\
\hline & UGC08605 & $\mathrm{J} 133654.3+32$ & 3035 & 5 & 9 & 15.60 & & & & & \\
\hline \multirow[t]{2}{*}{327} & NGC5247 & J133803.0-175303 & 1179 & 5 & 4 & 7.49 & 586 & 10.76 & 33.9 & 32.7 & 0.01 \\
\hline & ESO577-027 & J134246.9-193454 & 1232 & 5 & 10 & 11.56 & & & & & \\
\hline \multirow[t]{2}{*}{328} & NGC5219 & J133841.7-455121 & 2267 & 45 & 3 & 9.92 & 255 & 10.35 & 20.5 & 13.2 & 1.15 \\
\hline & HIPASS. & J133928.8-46 & 2306 & 9 & 8 & 13.83 & & & & & \\
\hline \multirow[t]{2}{*}{329} & NGC5303 & $\mathrm{J} 134745.0+381816$ & 1466 & 18 & 5 & 10.22 & 16 & 9.87 & 0.0 & & 0.39 \\
\hline & NGC5303B & J134745 & 1462 & 21 & 4 & 12.73 & & & & & \\
\hline \multirow[t]{2}{*}{330} & ESO383-091 & J135032.3-371720 & 857 & 5 & 7 & 11.02 & 34 & 9.04 & 121.6 & & 1.14 \\
\hline & $6 \mathrm{dF} \ldots$ & J135113.9-37 & 799 & 74 & 10 & 13.94 & & & & & \\
\hline \multirow[t]{2}{*}{331} & NGC5342 & $\mathrm{J} 135125.9+595148$ & 2368 & 27 & 1 & 10.14 & 192 & 10.29 & 18.6 & & 0.03 \\
\hline & SDSS... & $\mathrm{J} 134925.5+59$ & 2407 & 67 & 9 & 15.21 & & & & & \\
\hline \multirow[t]{2}{*}{332} & NGC5324 & J135205.9-060330 & 2922 & 5 & 5 & 9.36 & 122 & 10.78 & 0.7 & & 0.29 \\
\hline & LCRS.. & J135123.7-060413 & 2905 & 75 & 7 & 12.92 & & & & & \\
\hline \multirow[t]{2}{*}{333} & IC4341 & $\mathrm{J} 135334.2+373120$ & 2425 & 36 & 5 & 11.70 & 88 & 9.92 & 55.5 & 23.4 & 0.34 \\
\hline & UGC08795 & J135248.5+372927 & 2359 & 11 & 6 & 11.96 & & & & & \\
\hline \multirow[t]{2}{*}{334} & NGC5376 & J135516.1+593024 & 2230 & 31 & 5 & 9.09 & 458 & 10.69 & 10.4 & & 0.14 \\
\hline & UGC08741 & J134856.3+595009 & 2200 & 45 & 4 & 11.46 & & & & & \\
\hline \multirow[t]{2}{*}{335} & ESO510-013 & J135504.4-264650 & 3262 & 8 & 1 & 8.79 & 393 & 11.11 & 12.0 & & 1.61 \\
\hline & $6 \mathrm{dF} \ldots$ & J135543.9-261812 & 3320 & 74 & 5 & 13.06 & & & & & \\
\hline \multirow[t]{2}{*}{336} & VV100 & $\mathrm{J} 135545.5-060010$ & 1942 & 5 & 8 & 12.36 & 26 & 9.31 & 10.9 & & 1.47 \\
\hline & VV099 & J135534.2-055822 & 1915 & 52 & 9 & 13.88 & & & & & \\
\hline \multirow[t]{2}{*}{337} & NGC5377 & $\mathrm{J} 135616.7+471408$ & 1899 & 9 & 1 & 8.35 & 600 & 10.80 & 0.5 & & 0.19 \\
\hline & SDSS... & $\mathrm{J} 135005.0+462701$ & 1905 & 5 & 9 & 14.85 & & & & & \\
\hline \multirow[t]{2}{*}{338} & KUG1356... A & J135823.0+225317 & 2813 & 35 & 3 & 12.30 & 27 & 9.76 & 1.2 & & 0.97 \\
\hline & KUG1356... B & $\mathrm{J} 135824.7+225539$ & 2798 & 35 & 4 & 12.89 & & & & & \\
\hline \multirow[t]{2}{*}{339} & LCRS. & $\mathrm{J} 140125.2-035816$ & 3107 & 53 & 5 & 13.19 & 59 & 9.48 & 70.4 & & 1.80 \\
\hline & LCRS... & J140115.2-035414 & 3162 & 74 & 5 & 13.94 & & & & & \\
\hline \multirow[t]{2}{*}{340} & UGC08982 & $\mathrm{J} 140300.0+614504$ & 1868 & 31 & 9 & 12.84 & 153 & 9.09 & 30.5 & & 0.01 \\
\hline & SDSS... & J140524.6+613401 & 1883 & 5 & 9 & 14.41 & & & & & \\
\hline 341 & IC0971 & J140352.8-100826 & 3190 & 5 & 5 & 10.45 & 58 & 10.46 & 2.8 & & 2.26 \\
\hline
\end{tabular}


Table I: Table. (Contd.)

\begin{tabular}{|c|c|c|c|c|c|c|c|c|c|c|c|}
\hline No. & Name & J2000.0 & $\begin{array}{l}V_{L G} \\
\mathrm{~km}\end{array}$ & \begin{tabular}{|l|} 
\pm \\
-1
\end{tabular} & $\mathrm{~T}$ & $\begin{array}{r}K \\
\mathrm{mag}\end{array}$ & $\begin{array}{l}R_{\perp} \\
\mathrm{kpc}\end{array}$ & $\begin{array}{r}\log L \\
L_{\odot}\end{array}$ & $\begin{array}{r}\frac{M}{L_{K}} \\
\odot\end{array}$ & $\begin{array}{r}\frac{M^{c}}{L_{K}} \\
\odot\end{array}$ & $\log (I I)$ \\
\hline & IC4358 & J140334.2-100904 & 3156 & 75 & 4 & 12.67 & \multirow{3}{*}{18} & \multirow{3}{*}{10.16} & \multirow{3}{*}{0.0} & & \multirow{3}{*}{0.15} \\
\hline \multirow[t]{2}{*}{342} & ESO510-058 & J140437.4-244959 & 2156 & 8 & 6 & 10.43 & & & & & \\
\hline & ESO510-059 & J140446.4-244938 & 2160 & 8 & 6 & 12.14 & & & & & \\
\hline \multirow[t]{2}{*}{343} & UGC08995 & $\mathrm{J} 140447.4+084803$ & 1182 & 5 & 8. & 13.18 & \multirow[t]{2}{*}{8} & \multirow[t]{2}{*}{8.47} & \multirow[t]{2}{*}{42.0} & 38.9 & \multirow[t]{2}{*}{0.47} \\
\hline & LSBC. & J140441.6+084716 & 1145 & 5 & 10 & 16.34 & & & & & \\
\hline \multirow[t]{2}{*}{344} & NGC5468 & J140634.9-052711 & 2732 & 5 & 6 & 10.39 & \multirow[t]{2}{*}{55} & \multirow[t]{2}{*}{10.57} & \multirow[t]{2}{*}{8.7} & 4.6 & \multirow[t]{2}{*}{0.49} \\
\hline & NGC5 & J140655.0-052738 & 2803 & 32 & 2 & 10.64 & & & & & \\
\hline \multirow[t]{2}{*}{345} & HIPASS. & J140940.8-144622 & 2190 & 9 & 7 . & 13.21 & \multirow[t]{2}{*}{19} & \multirow[t]{2}{*}{9.16} & \multirow[t]{2}{*}{0.6} & & \multirow[t]{2}{*}{1.69} \\
\hline & 2MASX. & J140948.6-144721 & 2183 & 74 & 5. & 13.93 & & & & & \\
\hline \multirow[t]{2}{*}{346} & CGCG... & $\mathrm{J} 140954.9+564921$ & 1930 & 28 & 1 . & 11.58 & \multirow[t]{2}{*}{129} & \multirow[t]{2}{*}{9.56} & 30.9 & & 0.01 \\
\hline & SDSS... & J140942.0+563231 & 1903 & 73 & 5. & 14.11 & & & & & \\
\hline 347 & NGC5496 & J141137.9-010933 & 1455 & 5 & 7 . & 10.30 & 252 & 10.08 & 2.2 & & 0.31 \\
\hline & IC0976 & J14084 & 1445 & 15 & -1 & 10.33 & & & & & \\
\hline 348 & NGC5492 & J141035.2+193644 & 2274 & 7 & 1 & 10.17 & 392 & 10.24 & 17.2 & 13.4 & 1.61 \\
\hline & FGC1717 & $6+201836$ & 2299 & 6 & 8 & 15.03 & & & & & \\
\hline 349 & NGC5493 & J141129.4-050237 & 2609 & 30 & -2 & 8.45 & 616 & 11.04 & 1.5 & 0.3 & 0.21 \\
\hline & FGC1731 & $\mathrm{J} 141434.1-042502$ & 2594 & 5 & 7 & 12.53 & & & & & \\
\hline 350 & NGC5526 & J141353.7+574617 & 2180 & 16 & 6 . & 10.32 & 59 & 10.16 & 42.7 & 42.1 & 0.46 \\
\hline & MCG... & $\mathrm{J} 141312.5+574959$ & 2274 & 5 & 9 & 14.36 & & & & & \\
\hline 351 & NGC5585 & $\mathrm{J} 141948.2+564345$ & 458 & 7 & 7 & 9.49 & 81 & 9.13 & 20.5 & 13.3 & 0.03 \\
\hline & KUG1413... & $\mathrm{J} 141509.3+570515$ & 475 & 5 & 10 & 14.10 & & & & & \\
\hline 352 & NGC5582 & J142043.1+394137 & 1534 & 20 & -5 & 8.93 & 331 & 10.41 & 1.2 & & 0.47 \\
\hline & UGC09242 & $\mathrm{J} 142521.0+393222$ & 1525 & 9 & 7 & 11.73 & & & & & \\
\hline 353 & ESO385-012 & J142115.7-361336 & 3511 & 7 & 6 & 11.19 & 258 & 10.38 & 28.7 & 24.5 & 1.00 \\
\hline & ESO385-014 & $\mathrm{J} 142224.5-360118$ & 3464 & 10 & 4. & 11.86 & & & & & \\
\hline 354 & NGC5611 & J142404.8+330251 & 2025 & 46 & 1 & 9.75 & 61 & 10.33 & 28.2 & 27.1 & 2.18 \\
\hline & UGC09232 & $\mathrm{J} 142427.3+325707$ & 2116 & 5 & 8 & 13.82 & & & & & \\
\hline 355 & NGC5595 & J142413.2-164323 & 2560 & 47 & 5 & 9.15 & 42 & 10.91 & 0.4 & & 0.94 \\
\hline & NGC5597 & J142427.4-164546 & 2534 & 42 & 6 & 9.89 & & & & & \\
\hline 356 & NGC5624 & $\mathrm{J} 142635.2+513507$ & 2062 & 9 & 5. & 10.67 & 93 & 9.98 & 43.4 & 42.2 & 0.87 \\
\hline & SBS1423+517 & $\mathrm{J} 142524.6+513316$ & 2123 & 5 & 9 & 14.13 & & & & & \\
\hline 357 & NGC5630 & $\mathrm{J} 142736.6+411528$ & 2759 & 5 & 8 . & 10.84 & 324 & 10.14 & 0.1 & & 0.10 \\
\hline & SDSS... & $\mathrm{J} 142934.1+405602$ & 2757 & 34 & 9 & 14.92 & & & & & \\
\hline 358 & UGC09274 & J142802.8+211814 & 1117 & 73 & 7 . & 12.63 & 42 & 8.82 & 112.2 & & 1.96 \\
\hline & UGC09282 & $\mathrm{J} 142841.6+212022$ & 1155 & 5 & 10 & 13.36 & & & & & \\
\hline 359 & IC1014 & J142818.4+134649 & 1277 & 5 & 8 . & 11.36 & 70 & 9.33 & 0.0 & & 1.82 \\
\hline & UGC09273 & J142810.7+133306 & 1276 & 5 & 10 & 13.12 & & & & & \\
\hline 360 & CGCG... & J142914.5+444156 & 2538 & 46 & 1 . & 11.00 & 306 & 10.09 & 79.8 & & 0.34 \\
\hline
\end{tabular}


Table I: Table. (Contd.)

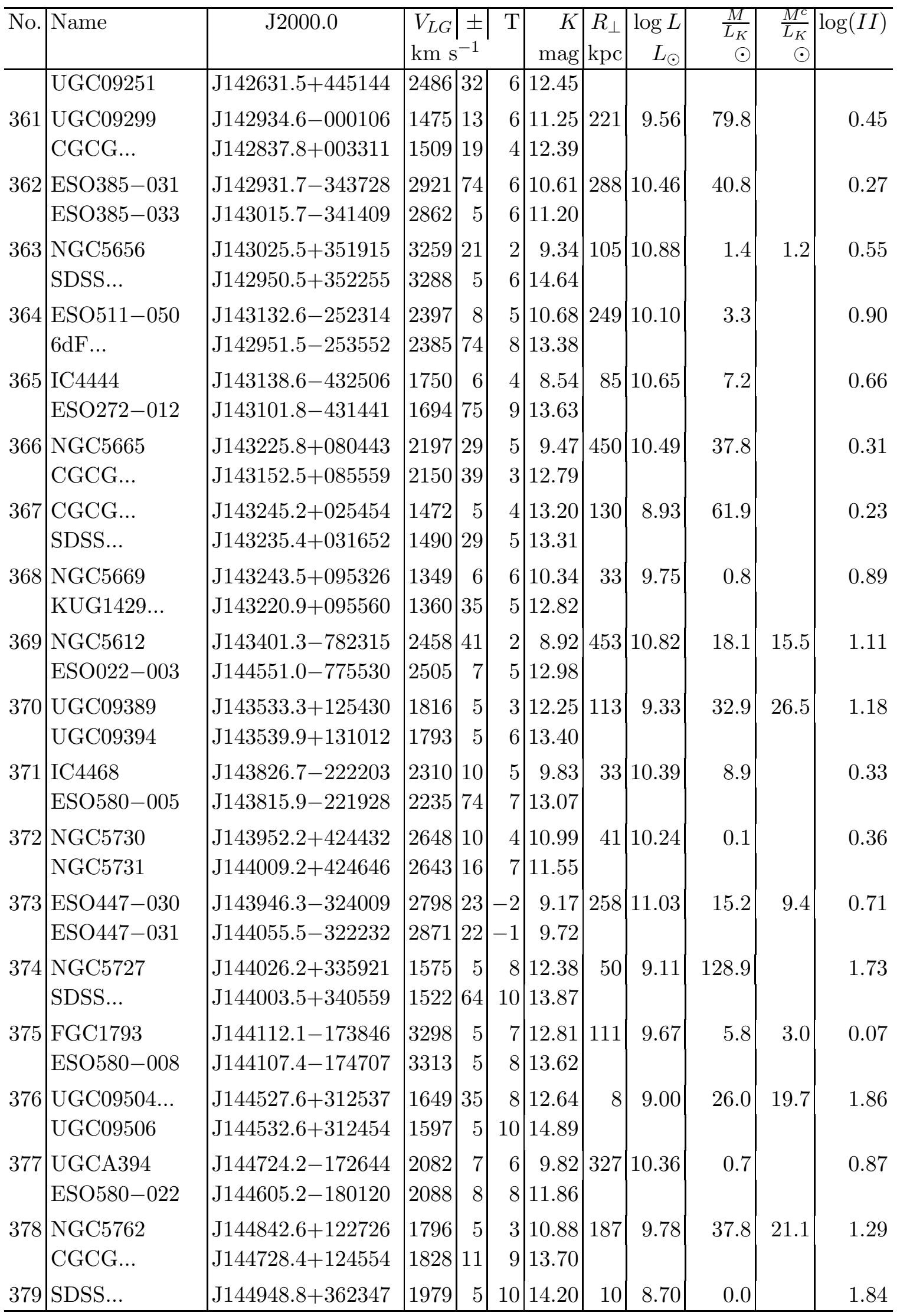


Table I: Table. (Contd.)

\begin{tabular}{|c|c|c|c|c|c|c|c|c|c|c|c|}
\hline No. & Name & J2000.0 & $\begin{array}{l}V_{L G} \\
\mathrm{~km} \mathrm{~s}\end{array}$ & $| \pm|$ & $\mathrm{T}$ & $\begin{array}{r}K \\
\mathrm{mag}\end{array}$ & $\begin{array}{l}R_{\perp} \\
\mathrm{kpc}\end{array}$ & $\begin{array}{r}\log L \\
L_{\odot}\end{array}$ & $\begin{array}{r}\frac{M}{L_{K}} \\
\odot\end{array}$ & $\begin{array}{r}\frac{M^{c}}{L_{K}} \\
\odot\end{array}$ & $\log (I I)$ \\
\hline & SDSS.. & J144951.1+362502 & 1978 & 32 & 10 & 14.79 & \multirow{3}{*}{21} & \multirow{3}{*}{9.19} & \multirow{3}{*}{41.7} & \multirow{3}{*}{11.5} & \multirow{3}{*}{2.33} \\
\hline \multirow[t]{2}{*}{380} & UGC09562 & J145114.4+353232 & 1355 & 21 & 5 & 12.04 & & & & & \\
\hline & UGC09560 & $\mathrm{J} 145056.5+353418$ & 1305 & 22 & 9 & 12.79 & & & & & \\
\hline \multirow[t]{2}{*}{381} & ESO512-023 & J145113.8-263753 & 2435 & 6 & 2 & 9.69 & \multirow[t]{2}{*}{9} & \multirow[t]{2}{*}{10.57} & \multirow[t]{2}{*}{6.9} & \multirow[t]{4}{*}{6.7} & \multirow[t]{2}{*}{0.79} \\
\hline & MCG.. & J145110.4-263818 & 2592 & 15 & 5 & 11.79 & & & & & \\
\hline \multirow[t]{2}{*}{382} & UGC0s & J145159.8+434314 & 2621 & 7 & 7 & 12.26 & \multirow[t]{2}{*}{54} & \multirow[t]{2}{*}{9.80} & \multirow[t]{2}{*}{16.9} & & \multirow[t]{2}{*}{1.57} \\
\hline & UGC0؟ & $\mathrm{J} 145146.5+433841$ & 2662 & 29 & 6 & 12.41 & & & & & \\
\hline \multirow[t]{2}{*}{383} & CGCG... & $\mathrm{J} 145601.2+022749$ & 2082 & 24 & -2 & 11.56 & \multirow[t]{2}{*}{191} & \multirow[t]{2}{*}{9.62} & \multirow[t]{2}{*}{98.5} & & \multirow[t]{2}{*}{0.46} \\
\hline & SDSS. & $\mathrm{J} 145434.4+022038$ & 2124 & 42 & 10 & 15.27 & & & & & \\
\hline \multirow[t]{2}{*}{384} & NGC5798 & $\mathrm{J} 145738.0+295807$ & 1870 & 7 & 9 & 10.94 & \multirow[t]{2}{*}{156} & 10.00 & 4.8 & 2.1 & 0.58 \\
\hline & NGC5789 & $\mathrm{J} 145635.5+301403$ & 1886 & 5 & 8 & 11.23 & & & & & \\
\hline 385 & UGC09665 & $\mathrm{J} 150132.5+481911$ & 2707 & 7 & 4 & 10.26 & 82 & 10.38 & 4.0 & 3.3 & 1.11 \\
\hline & UGC0 & J150048 & 2675 & 7 & 8 & 12.87 & & & & & \\
\hline 386 & IC4522 & J151128.9-755136 & 2617 & 9 & 3 & 9.58 & 408 & 10.62 & 32.3 & 28.6 & 0.73 \\
\hline & $2 \mathrm{MASX} \ldots$ & $\mathrm{J} 150622.8-7$ & 2670 & 9 & 6 & 12.56 & & & & & \\
\hline 387 & 2MASXi... & J151156.7+323553 & 2323 & 5 & 9 & 14.31 & 89 & 8.83 & 8.6 & & 0.16 \\
\hline & UGC09762:... & $\mathrm{J} 151154.0+324530$ & 2330 & 5 & 10 & 14.67 & & & & & \\
\hline 388 & ESO581-025 & J151330.9-204030 & 2165 & 9 & 4 & 9.06 & 45 & 10.63 & 2.1 & & 0.70 \\
\hline & $2 \mathrm{MAS}$ & J151338.2-204529 & 2124 & 74 & 5 & 13.34 & & & & & \\
\hline 389 & NGC5878 & J151345.7-141611 & 1908 & 5 & 3 & 8.12 & 524 & 10.90 & 17.9 & 15.5 & 0.52 \\
\hline & MCG.. & $\mathrm{J} 151354.2-130622$ & 1860 & 9 & 3 & 11.85 & & & & & \\
\hline 390 & VIIZw 591 & $\mathrm{~J} 151504.1+611212$ & 2692 & 26 & 3 & 10.89 & 331 & 10.12 & 28.2 & & 0.40 \\
\hline & MCG... & $\mathrm{J} 151051.0+610852$ & 2723 & 18 & 9 & 13.82 & & & & & \\
\hline 391 & NGC5899 & $\mathrm{J} 151503.3+420259$ & 2726 & 20 & 5 & 8.57 & 103 & 11.18 & 4.7 & 4.2 & 0.40 \\
\hline & NGC5900 & $\mathrm{J} 151505.1+421235$ & 2649 & 8 & 3 & 9.42 & & & & & \\
\hline 392 & NGC5917 & J152132.6-072238 & 1855 & 36 & 3 & 10.74 & 32 & 10.04 & 3.8 & & 1.19 \\
\hline & MCG... & J152133.3-072652 & 1888 & 75 & -2 & 11.26 & & & & & \\
\hline 393 & MCG... & J152135.9-120536 & 3156 & 59 & 1 & 9.50 & 617 & 10.80 & 140.1 & & 0.64 \\
\hline & $6 \mathrm{dF} \ldots$ & J152224.4-125219 & 3267 & 74 & 7 & 13.95 & & & & & \\
\hline 394 & MRK0482 & $\mathrm{J} 152804.2+553245$ & 3515 & 38 & 2 & 11.19 & 95 & 10.27 & 8.0 & & 0.32 \\
\hline & MRK0481 & $\mathrm{J} 152750.5+552613$ & 3479 & 75 & -1 & 13.00 & & & & & \\
\hline 395 & IC1125 & J153305.6-013742 & 2773 & 12 & 8 & 10.88 & 76 & 10.14 & 48.7 & 10.4 & 0.30 \\
\hline & SDSS... & $\mathrm{J} 153239.2-013606$ & 2859 & 39 & 9 & 15.30 & & & & & \\
\hline 396 & NGC5957 & $\mathrm{J} 153523.2+120250$ & 1871 & 5 & 3 & 9.48 & 143 & 10.59 & 23.0 & 22.6 & 0.09 \\
\hline & NGC5956 & $\mathrm{J} 153458.5+114501$ & 1944 & 5 & 2 & 9.86 & & & & & \\
\hline 397 & NGC5970 & $\mathrm{J} 153830.0+121112$ & 2003 & 5 & 5 & 8.80 & 67 & 10.73 & 5.3 & 1.7 & 0.09 \\
\hline & IC1131 & $\mathrm{J} 153851.7+120450$ & 2063 & 26 & -5 & 11.05 & & & & & \\
\hline 398 & ESO022-010 & J153334.9-780726 & 2453 & 59 & -2 & 10.13 & 444 & 10.53 & 2.2 & & 1.17 \\
\hline
\end{tabular}


Table I: Table. (Contd.)

\begin{tabular}{|c|c|c|c|c|c|c|c|c|c|c|c|}
\hline No. & Name & J2000.0 & \begin{tabular}{|l|}
$V_{L G}$ \\
$\mathrm{~km} \mathrm{~s}$
\end{tabular} & & $\mathrm{~T}$ & $\begin{array}{r}K \\
\mathrm{mag}\end{array}$ & $\begin{array}{l}R_{\perp} \\
\mathrm{kpc}\end{array}$ & $\begin{array}{r}\log L \\
L_{\odot}\end{array}$ & $\begin{array}{r}\frac{M}{L_{K}} \\
\odot\end{array}$ & $\begin{array}{r}\frac{M^{c}}{L_{K}} \\
\odot\end{array}$ & $\log (I I)$ \\
\hline \multirow{3}{*}{399} & IC4555 & J154814.8-781044 & 2465 & 10 & 6 & 10.64 & \multirow{3}{*}{117} & \multirow{3}{*}{10.16} & \multirow{3}{*}{19.6} & \multirow{3}{*}{18.7} & \multirow{3}{*}{0.96} \\
\hline & UGC09977 & $\mathrm{J} 154159.5+004246$ & 1918 & 5 & 5 & 10.56 & & & & & \\
\hline & UGC09979 & $\mathrm{J} 154219.5+002828$ & 1964 & 5 & 10 & 10.98 & & & & & \\
\hline \multirow[t]{2}{*}{400} & UGC10010 & $\mathrm{J} 154445.7+460441$ & 2805 & 23 & 7 & 12.54 & \multirow[t]{2}{*}{212} & \multirow[t]{2}{*}{9.67} & \multirow[t]{2}{*}{41.5} & & \multirow[t]{2}{*}{1.01} \\
\hline & MRK0490 & $\mathrm{J} 154630.7+455954$ & 2832 & 5 & 9 & 13.09 & & & & & \\
\hline \multirow[t]{2}{*}{401} & UGC10043 & $\mathrm{J} 154841.2+215210$ & 2252 & 5 & 4 & 10.39 & \multirow[t]{2}{*}{79} & \multirow[t]{2}{*}{10.16} & \multirow[t]{2}{*}{54.5} & & \multirow[t]{2}{*}{1.14} \\
\hline & UGC10049. & J154917.1+214943 & 2343 & 63 & 8 & 14.39 & & & & & \\
\hline \multirow[t]{2}{*}{402} & NGC6015 & $\mathrm{J} 155125.2+621836$ & 1041 & 6 & 6 & 8.47 & \multirow[t]{2}{*}{257} & \multirow[t]{2}{*}{10.26} & \multirow[t]{2}{*}{50.1} & 48.1 & \multirow[t]{2}{*}{0.28} \\
\hline & UGC10031 & $\mathrm{J} 154545.7+613321$ & 1096 & 6 & 9 & 14.59 & & & & & \\
\hline \multirow[t]{2}{*}{403} & NGC6017 & $\mathrm{J} 155715.5+055954$ & 1830 & 75 & -2 & 9.79 & \multirow[t]{2}{*}{120} & \multirow[t]{2}{*}{10.20} & 6.6 & & 0.15 \\
\hline & SDSS.. & $\mathrm{J} 155614.4+060553$ & 1857 & 45 & 9 & 14.70 & & & & & \\
\hline 404 & NGC6070 & J160958.7+004234 & 2033 & 7 & 6 & 8.69 & 556 & 10.76 & 2.9 & 1.2 & 0.02 \\
\hline & UGC10290 & $\mathrm{J} 161432.9+004918$ & 2017 & 6 & 9 & 11.40 & & & & & \\
\hline 405 & UGC10288 & J161424.8-001227 & 2075 & 5 & 5 & 9.34 & 358 & 10.51 & 24.5 & 23.2 & 0.02 \\
\hline & VV370 & J161329.0-005301 & 2118 & 5 & 9 & 12.55 & & & & & \\
\hline 406 & MCG... & J164203.2-050158 & 1601 & 5 & 4 & 8.99 & 383 & 10.46 & 2.2 & 0.6 & 1.42 \\
\hline & MCG... & J163808.6-044924 & 1612 & 5 & 4 & 11.07 & & & & & \\
\hline 407 & NGC6207 & $\mathrm{J} 164303.7+364957$ & 1037 & 5 & 3 & 9.12 & 293 & 9.98 & 0.5 & & 0.76 \\
\hline & UGC10477 & $\mathrm{J} 163734.8+371710$ & 1033 & 8 & 7 & 13.25 & & & & & \\
\hline 408 & 2MASX... & J164808.3-002514 & 2396 & 74 & 6 & 12.52 & 57 & 9.62 & 1.7 & & 2.63 \\
\hline & $2 \mathrm{MASX} \ldots$ & J164759.2-001945 & 2407 & 9 & 10 & 12.62 & & & & & \\
\hline 409 & UGC10625 & J165723.1+384019 & 2252 & 6 & 8 & 13.82 & 15 & 8.97 & 0.2 & & 1.02 \\
\hline & SHOC553 & $\mathrm{J} 165730.0+384123$ & 2255 & 5 & 9 & 14.37 & & & & & \\
\hline 410 & UGC10743 & $\mathrm{J} 171130.7+075941$ & 2683 & 20 & 3 & 10.32 & 285 & 10.33 & 8.9 & 6.7 & 2.08 \\
\hline & HIPASS... & $\mathrm{J} 170956.0+074713$ & 2707 & 5 & 8 & 14.06 & & & & & \\
\hline 411 & UGC10770... & $\mathrm{J} 171310.2+591956$ & 1391 & 32 & 8 & 11.60 & 4 & 9.54 & 0.6 & & 2.52 \\
\hline & UGC10770.. & $\mathrm{J} 171307.1+591924$ & 1414 & 27 & 8 & 11.60 & & & & & \\
\hline 412 & NGC6368 & $\mathrm{J} 172711.5+113237$ & 2905 & 5 & 3 & 9.13 & 221 & 10.87 & 0.7 & 0.4 & 0.06 \\
\hline & UGC10852 & $\mathrm{J} 172617.3+111901$ & 2919 & 5 & 6 & 13.53 & & & & & \\
\hline 413 & UGC10887 & $\mathrm{J} 172607.0+774213$ & 2088 & 5 & 6 & 12.32 & 185 & 9.57 & 18.3 & & 1.84 \\
\hline & UGC10907 & $\mathrm{J} 172959.2+772347$ & 2071 & 34 & 4 & 12.46 & & & & & \\
\hline 414 & UGC10864 & $\mathrm{J} 172819.4+141008$ & 3030 & 31 & -2 & 10.12 & 158 & 10.55 & 66.5 & 60.1 & 1.20 \\
\hline & CGCG... & $\mathrm{J} 172901.7+141751$ & 3143 & 16 & 3 & 12.88 & & & & & \\
\hline 415 & NGC6384 & $\mathrm{J} 173224.3+070337$ & 1791 & 5 & 4 & 7.48 & 481 & 11.11 & 2.7 & 2.3 & 1.65 \\
\hline & UGC10862 & $\mathrm{J} 172808.9+072521$ & 1816 & 5 & 5 & 11.66 & & & & & \\
\hline 416 & NGC6470 & $\mathrm{J} 174414.9+673710$ & 1719 & 21 & 3 & 11.12 & 145 & 9.70 & 0.0 & & 2.09 \\
\hline & UGC10991 & $\mathrm{J} 174628.9+672017$ & 1718 & 5 & 10 & 12.72 & & & & & \\
\hline 417 & ESO139-049 & J180035.0-590811 & 2611 & 8 & 6 & 10.25 & 257 & 10.44 & 40.6 & 36.6 & 0.42 \\
\hline
\end{tabular}


Table I: Table. (Contd.)

\begin{tabular}{|c|c|c|c|c|c|c|c|c|c|c|c|}
\hline No. & Name & $\mathrm{J} 2000.0$ & $\begin{array}{l}V_{L G} \\
\mathrm{~km} \mathrm{~s}\end{array}$ & \begin{tabular}{|l|} 
\pm \\
-1
\end{tabular} & $\mathrm{~T}$ & $\begin{array}{r}K \\
\mathrm{mag}\end{array}$ & $\begin{array}{l}R_{\perp} \\
\mathrm{kpc}\end{array}$ & $\begin{array}{r}\log L \\
L_{\odot}\end{array}$ & $\begin{array}{r}\frac{M}{L_{K}} \\
\odot\end{array}$ & $\begin{array}{r}\frac{M^{c}}{L_{K}} \\
\odot\end{array}$ & $\log (I I)$ \\
\hline & ESO139-046 & J175916.9-593026 & 2672 & 10 & 6 & 11.55 & \multirow{3}{*}{615} & \multirow{3}{*}{11.04} & \multirow{3}{*}{15.7} & \multirow{3}{*}{10.5} & \multirow{3}{*}{0.38} \\
\hline \multirow[t]{2}{*}{418} & NGC6548 & $\mathrm{J} 180559.2+183514$ & 2369 & 27 & 1 & 8.55 & & & & & \\
\hline & NGC6555 & $\mathrm{J} 180749.2+173618$ & 2418 & 5 & 5 & 9.80 & & & & & \\
\hline \multirow[t]{2}{*}{419} & 2MASX. & J181037.5-561640 & 3459 & 36 & 5. & 10.67 & \multirow[t]{2}{*}{92} & \multirow[t]{2}{*}{10.65} & \multirow[t]{2}{*}{4.5} & & \multirow[t]{2}{*}{1.15} \\
\hline & IC4679 & J181124.5-561516 & 3502 & 16 & 6 . & 10.92 & & & & & \\
\hline \multirow[t]{2}{*}{420} & NGC6574 & $\mathrm{J} 181151.2+145854$ & 2471 & 6 & 4 & 8.35 & \multirow[t]{2}{*}{535} & \multirow[t]{2}{*}{11.13} & \multirow[t]{2}{*}{0.1} & & \multirow[t]{2}{*}{0.14} \\
\hline & NGC6 & J181107.3 & 2466 & 6 & 9 & 9.82 & & & & & \\
\hline \multirow[t]{2}{*}{421} & NGC6585 & J181221.8+393759 & 3077 & 8 & 4 . & 10.26 & \multirow[t]{2}{*}{380} & \multirow[t]{2}{*}{10.48} & \multirow[t]{2}{*}{2.7} & \multirow[t]{4}{*}{1.1} & \multirow[t]{2}{*}{0.29} \\
\hline & UGC1 & J181109.3 & 3064 & 5 & 6 . & 13.37 & & & & & \\
\hline \multirow[t]{2}{*}{422} & UGC11152 & $\mathrm{J} 181232.2+183556$ & 2928 & 5 & 8 . & 12.56 & \multirow[t]{2}{*}{28} & 9.54 & 8.6 & & 0.89 \\
\hline & UGC11 & $\mathrm{J} 181222.5$ & 2958 & 20 & 10 & 15.15 & & & & & \\
\hline 423 & IC4694 & J181526.4-581232 & 2644 & 9 & 5 & 10.29 & 265 & 10.60 & 31.6 & 9.8 & 0.88 \\
\hline & $2 \mathrm{MAs}^{\mathrm{s}}$ & J181214. & 2580 & 36 & 1 & 10.31 & & & & & \\
\hline 424 & NGC6599 & $\mathrm{J} 181543.0+245445$ & 3253 & 26 & -2 & 9.27 & 183 & 11.12 & 3.5 & & 1.03 \\
\hline & NGC6 & J181634. & 3299 & 25 & 2 & 9.77 & & & & & \\
\hline 425 & NGC6438 & J182217.5-852407 & 2349 & 75 & -2 & 8.22 & 4 & 11.29 & 0.0 & & 0.02 \\
\hline & NGC6438A & $\mathrm{J} 182235.5-85$ & 2377 & 75 & 10 & 8.55 & & & & & \\
\hline 426 & NGC6667 & J183039.8+675913 & 2856 & 6 & 2 & 9.28 & 569 & 10.82 & 16.9 & & 2.18 \\
\hline & UGC11222 & $\mathrm{J} 182156.4+68$ & 2897 & 63 & 6 & 12.32 & & & & & \\
\hline 427 & IC4721 & J183424.8-582948 & 2116 & 5 & 6 & 8.90 & 71 & 10.77 & 45.5 & 45.0 & 1.30 \\
\hline & IC4720 & J183332.5-5 & 1936 & 11 & 6 & 10.00 & & & & & \\
\hline 428 & UGC11291 & $\mathrm{J} 183604.5+305023$ & 3137 & 7 & 7 . & 13.17 & 225 & 9.50 & 24.3 & & 0.17 \\
\hline & CGCG... & $\mathrm{J} 183506.2+303730$ & 3154 & 29 & 5 & 13.85 & & & & & \\
\hline 429 & NGC6654A & $\mathrm{J} 183927.1+733442$ & 1824 & 7 & 7 . & 11.91 & 19 & 9.58 & 1.0 & & 0.23 \\
\hline & UGC11331 & $\mathrm{J} 183860.0+7 \mathrm{~s}$ & 1811 & 9 & 10 & 12.26 & & & & & \\
\hline 430 & IC4807 & J190217.7-565552 & 3397 & 36 & 5 & 10.53 & 281 & 10.47 & 19.1 & 10.3 & 0.29 \\
\hline & HIPASS... & J185949.7-570128 & 3356 & 9 & 6 . & 12.98 & & & & & \\
\hline 431 & NGC6764 & J190816.4+505560 & 2700 & 8 & 4 & 9.33 & 405 & 10.81 & 2.5 & & 1.49 \\
\hline & NGC6759 & J190656.8+502039 & 2718 & 45 & 2 & 10.95 & & & & & \\
\hline 432 & IC4837 & J191514.6-543947 & 2582 & 18 & 6 & 9.80 & 555 & 10.70 & 6.1 & & 0.30 \\
\hline & IC4821 & J190932.0-550102 & 2603 & 10 & 5 & 10.35 & & & & & \\
\hline 433 & ESO338-004 & J192758.2-413432 & 2850 & 26 & 4 & 12.60 & 76 & 9.64 & 57.2 & & 1.62 \\
\hline & ESO338-004B & J192731.6-413854 & 2903 & 75 & 5 & 13.32 & & & & & \\
\hline 434 & NGC6814 & J194240.6-101925 & 1703 & 5 & 4 & 7.59 & 617 & 11.00 & 23.3 & 21.0 & 0.74 \\
\hline & HIPASS... & J194833.7-094802 & 1647 & 9 & 10 & 13.89 & & & & & \\
\hline 435 & NGC6810 & J194334.4-583921 & 1929 & 46 & 2 & 8.66 & 86 & 10.68 & 19.6 & 17.9 & 0.19 \\
\hline & ESO142-032 & J194236.9-584806 & 1833 & 13 & 10 & 12.88 & & & & & \\
\hline 436 & NGC6835 & J195433.1-123409 & 1761 & 54 & 1 & 8.80 & 51 & 10.61 & 0.0 & & 0.79 \\
\hline
\end{tabular}


Table I: Table. (Contd.)

\begin{tabular}{|c|c|c|c|c|c|c|c|c|c|c|c|}
\hline \multirow[t]{2}{*}{ No. } & Name & J2000.0 & $\begin{array}{l}V_{L G} \\
\mathrm{~km} \mathrm{~s}\end{array}$ & \begin{tabular}{|l|} 
\pm \\
-1
\end{tabular} & $\mathrm{~T}$ & $\begin{array}{r}K \\
\mathrm{mag}\end{array}$ & $\begin{array}{l}R_{\perp} \\
\mathrm{kpc}\end{array}$ & $\begin{array}{r}\log L \\
L_{\odot}\end{array}$ & $\begin{array}{r}\frac{M}{L_{K}} \\
\odot\end{array}$ & $\begin{array}{r}\frac{M^{c}}{L_{K}} \\
\odot\end{array}$ & $\log (I I)$ \\
\hline & NGC6836 & $195440.1-124117$ & 1763 & 5 & 9 & 11.11 & \multirow{3}{*}{679} & \multirow{3}{*}{11.08} & \multirow{3}{*}{43.8} & \multirow{3}{*}{40.3} & \multirow{3}{*}{0.74} \\
\hline \multirow[t]{2}{*}{437} & NGC6869 & $200042.4+661339$ & 3024 & 42 & -2 & 8.65 & & & & & \\
\hline & kkh092 & $\mathrm{J} 201001.1+660501$ & 2943 & 11 & 10 & 14.21 & & & & & \\
\hline \multirow[t]{2}{*}{438} & HIPASS... & J201046.9-113835 & 3352 & 9 & 7 . & 12.28 & \multirow[t]{2}{*}{55} & \multirow[t]{2}{*}{9.88} & \multirow[t]{2}{*}{75.8} & & \multirow[t]{2}{*}{0.60} \\
\hline & $2 \mathrm{MASX} .$. & J201103.7-113808 & 3258 & 74 & 5. & 13.13 & & & & & \\
\hline \multirow[t]{2}{*}{439} & 2MASX. & J201411.8-114537 & 3385 & 74 & 7 . & 11.73 & \multirow[t]{2}{*}{276} & \multirow[t]{2}{*}{10.05} & \multirow[t]{2}{*}{3.0} & & \multirow[t]{2}{*}{0.60} \\
\hline & HIPASS... & J201252.5-113913 & 3395 & 9 & 5. & 13.29 & & & & & \\
\hline \multirow[t]{2}{*}{440} & IC4960 & J201523.9-703216 & 3324 & 30 & -2 & 10.42 & \multirow[t]{2}{*}{474} & \multirow[t]{2}{*}{10.51} & \multirow[t]{2}{*}{52.7} & \multirow[t]{2}{*}{13.5} & \multirow[t]{2}{*}{0.13} \\
\hline & IC4962 & J201642.5-710746 & 3269 & 5 & 4. & 12.55 & & & & & \\
\hline \multirow[t]{2}{*}{441} & NGC6887 & J201717.3-524748 & 2639 & 8 & 4 & 8.85 & 117 & 10.89 & 0.3 & & 0.63 \\
\hline & NGC6887: & J201605.2-524539 & 2626 & 30 & 9 & 14.00 & & & & & \\
\hline 442 & IC1313 & J201843.7-165645 & 3421 & 19 & 2 & 9.56 & 60 & 10.83 & 30.9 & 8.6 & 0.31 \\
\hline & $2 \mathrm{MASX} \ldots$ & J201828.6-16 & 3250 & 74 & 3 & 12.93 & & & & & \\
\hline 443 & ESO $462-025$ & $\mathrm{~J} 202908.2-275452$ & 3127 & 5 & 8 . & 13.28 & 215 & 9.42 & 203.8 & & 0.87 \\
\hline & ESO462-022 & J202806.0-280505 & 3173 & 68 & 8 & 14.24 & & & & & \\
\hline 444 & IC5020 & J203038.5-332908 & 3127 & 8 & 3 & 9.26 & 91 & 10.88 & 0.1 & & 0.18 \\
\hline & ESO400-037 & J203113.5-332841 & 3119 & 5 & 6 & 13.72 & & & & & \\
\hline 445 & ESO596-051 & J203105.0-181202 & 2413 & 74 & 7 . & 11.41 & 3 & 9.89 & 0.0 & & 2.74 \\
\hline & ESO596-051 & J203106.3-181157 & 2414 & 9 & 8 & 12.87 & & & & & \\
\hline 446 & AM2029-235... & J203236.0-234258 & 3154 & 74 & 3 & 13.61 & 35 & 9.43 & 0.3 & & 0.65 \\
\hline & AM2029-235. & $\mathrm{J} 203225.5-234135$ & 3158 & 74 & 3 & 13.67 & & & & & \\
\hline 447 & ESO234-049 & J203518.1-495156 & 2536 & 8 & 4 . & 10.59 & 372 & 10.17 & 7.3 & & 0.31 \\
\hline & $6 \mathrm{dF} \ldots$ & J203210.1-493056 & 2520 & 74 & 9 & 14.23 & & & & & \\
\hline 448 & ESO186-065 & J203557.2-541758 & 3398 & 26 & -3 & 9.93 & 332 & 10.70 & 16.3 & & 0.28 \\
\hline & IC5021 & J203334.0-543115 & 3353 & 74 & 7 . & 12.92 & & & & & \\
\hline 449 & ESO234-050 & J203557.9-501132 & 2650 & 75 & -1 & 11.19 & 125 & 9.96 & 0.1 & & 0.31 \\
\hline & ESO234-056 & $\mathrm{J} 203702.2-500538$ & 2648 & 65 & 8 & 15.91 & & & & & \\
\hline 450 & IC5039 & J204314.3-295112 & 2763 & 7 & 4 & 9.88 & 110 & 10.78 & 0.9 & 0.6 & 0.66 \\
\hline & IC5007 & J204334.4-294213 & 2783 & 5 & 7 . & 10.09 & & & & & \\
\hline 451 & NGC6920 & J204357.4-800003 & 2699 & 40 & -2 & 8.28 & 75 & 11.13 & 15.6 & 1.4 & 0.36 \\
\hline & ESO026-005 & J204601.4-800451 & 2545 & 75 & 9 & 11.40 & & & & & \\
\hline 452 & ESO285-048 & $\mathrm{J} 204440.2-455843$ & 2693 & 5 & 6 . & 10.90 & 173 & 10.13 & 7.5 & & 0.32 \\
\hline & ESO285-051 & J204600.8-455059 & 2715 & 35 & 9 & 13.28 & & & & & \\
\hline 453 & NGC6958 & J204842.6-375951 & 2740 & 25 & -4 & 8.38 & 138 & 11.11 & 1.6 & & 0.21 \\
\hline & $2 \mathrm{MASX} .$. & J204935.7-375240 & 2705 & 40 & -3 & 12.39 & & & & & \\
\hline 454 & NGC7029 & J211152.1-491701 & 2750 & 37 & -5 & 8.53 & 702 & 11.06 & 0.0 & & 0.55 \\
\hline & ESO235-073 & $\mathrm{J} 211157.5-502105$ & 2750 & 9 & 8. & 12.79 & & & & & \\
\hline 455 & NGC7070A & $\mathrm{J} 213147.3-425052$ & 2385 & 26 & 0 & 9.30 & 198 & 10.80 & 0.2 & & 0.59 \\
\hline
\end{tabular}


Table I: Table. (Contd.)

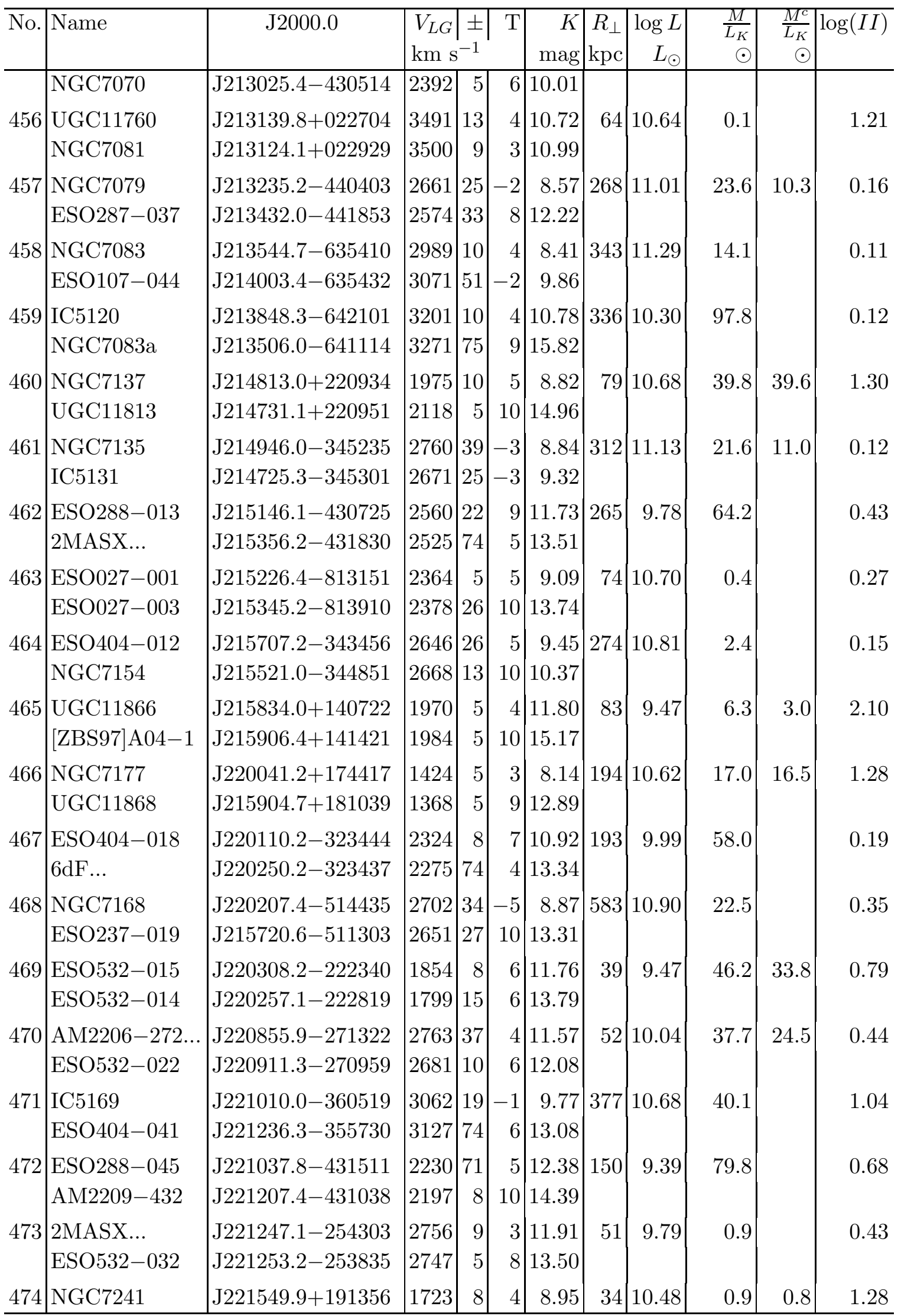


Table I: Table. (Contd.)

\begin{tabular}{|c|c|c|c|c|c|c|c|c|c|c|c|}
\hline No. & Name & J2000.0 & \begin{tabular}{|l|}
$V_{L G}$ \\
$\mathrm{~km} \mathrm{~s}$ \\
\end{tabular} & $\mid$ & $\mathrm{T}$ & $\begin{array}{r}K \\
\mathrm{mag}\end{array}$ & $\begin{array}{l}R_{\perp} \\
\mathrm{kpc}\end{array}$ & $\begin{array}{r}\log L \\
L_{\odot}\end{array}$ & $\begin{array}{r}\frac{M}{L_{K}} \\
\odot \\
\end{array}$ & $\begin{array}{r}\frac{M^{c}}{L_{K}} \\
\odot\end{array}$ & $\log (I I)$ \\
\hline & UGC11964 & J221529.1+191312 & 1697 & 5 & 7 & 12.92 & & & & & \\
\hline \multirow[t]{2}{*}{475} & 2MASX... & $\mathrm{J} 221805.4-253116$ & 2827 & 10 & 3 & 11.86 & \multirow[t]{2}{*}{113} & \multirow[t]{2}{*}{9.83} & \multirow[t]{2}{*}{0.1} & & \multirow[t]{2}{*}{1.46} \\
\hline & ESO533-010 & $\mathrm{J} 221807.0-254120$ & 2825 & 5 & 9 & 13.57 & & & & & \\
\hline \multirow[t]{2}{*}{476} & NGC7259 & $\mathrm{J} 222305.5-285717$ & 1789 & 37 & 3 & 10.75 & \multirow[t]{2}{*}{21} & \multirow[t]{2}{*}{9.83} & \multirow[t]{2}{*}{29.1} & 27.6 & \multirow[t]{2}{*}{1.31} \\
\hline & ESO467-051 & J222316.4-285848 & 1879 & 8 & 6 & 14.21 & & & & & \\
\hline \multirow[t]{2}{*}{477} & MCG. & J222741.5-094337 & 1786 & 43 & 8 & 13.10 & \multirow[t]{2}{*}{33} & \multirow[t]{2}{*}{9.08} & \multirow[t]{2}{*}{208.3} & & \multirow[t]{2}{*}{2.64} \\
\hline & $6 \mathrm{dF} \ldots$ & J222730.5-093959 & 1866 & 74 & 9 & 13.59 & & & & & \\
\hline \multirow[t]{2}{*}{478} & NGC7307 & $\mathrm{J} 223352.5-405558$ & 2088 & 5 & 6 & 10.41 & \multirow[t]{2}{*}{158} & \multirow[t]{2}{*}{10.12} & \multirow[t]{2}{*}{113.5} & & \multirow[t]{2}{*}{0.95} \\
\hline & ESO345-027 & $\mathrm{J} 223414.4-411404$ & 2178 & 75 & 8 & 12.85 & & & & & \\
\hline \multirow[t]{2}{*}{479} & NGC7314 & $\mathrm{J} 223546.2-260301$ & 1511 & 5 & 4 & 8.18 & \multirow[t]{2}{*}{99} & \multirow[t]{2}{*}{10.67} & \multirow[t]{2}{*}{1.2} & 0.9 & 1.45 \\
\hline & ESO534-001 & $\mathrm{J} 223607.0-261852$ & 1490 & 5 & 9 & 13.33 & & & & & \\
\hline 480 & ESO534-010 & $\mathrm{J} 223851.6-254233$ & 3207 & 42 & -3 & 9.94 & 371 & 10.62 & 71.3 & & 0.27 \\
\hline & ESO534-015 & $\mathrm{J} 224000.8-260728$ & 3125 & 74 & 2 & 14.18 & & & & & \\
\hline 481 & ESO534-021 & $\mathrm{J} 224434.7-225931$ & 3247 & 13 & 5 & 11.65 & 314 & 10.08 & 5.6 & & 0.36 \\
\hline & $2 \mathrm{MASX} .$. & J224419.6-232329 & 3261 & 31 & 7 & 12.82 & & & & & \\
\hline 482 & NGC7416 & J225541.7-052943 & 3031 & 10 & 3 & 9.23 & 654 & 10.86 & 12.0 & & 1.85 \\
\hline & MCG... & $\mathrm{J} 225834.5-045618$ & 2998 & 74 & 6 & 13.82 & & & & & \\
\hline 483 & IC5273 & $\mathrm{J} 225926.7-374210$ & 1310 & 9 & 6 & 8.75 & 164 & 10.31 & 21.6 & 20.1 & 0.27 \\
\hline & ESO406-040 & $\mathrm{J} 230022.2-371205$ & 1263 & 6 & 10 & 14.15 & & & & & \\
\hline 484 & NGC7448 & $\mathrm{J} 230003.6+155849$ & 2449 & 6 & 5 & 8.99 & 317 & 10.79 & 6.5 & 5.9 & 0.05 \\
\hline & UGC12321 & $\mathrm{J} 230219.0+160140$ & 2417 & 5 & 4 & 12.07 & & & & & \\
\hline 485 & NGC7457 & $\mathrm{J} 230059.9+300842$ & 1119 & 11 & -3 & 8.17 & 36 & 10.46 & 12.7 & 12.3 & 0.32 \\
\hline & UGC12311 & $\mathrm{J} 230125.1+301421$ & 1211 & 8 & 6 & 12.34 & & & & & \\
\hline 486 & NGC7456 & J230210.4-393410 & 1212 & 8 & 6 & 9.35 & 310 & 10.03 & 4.2 & & 0.35 \\
\hline & LCRS... & J230153.2-382941 & 1201 & 30 & 10 & 12.31 & & & & & \\
\hline 487 & ESO027-021 & J230419.1-792757 & 2308 & 20 & 1 & 10.56 & 46 & 10.13 & 8.4 & 6.2 & 0.74 \\
\hline & {$[$ SOM2000]... } & J230415.6-793258 & 2262 & 10 & 9 & 12.75 & & & & & \\
\hline 488 & UGC12347 & $\mathrm{J} 230512.2+185205$ & 1899 & 21 & 10 & 12.32 & 78 & 9.43 & 0.7 & & 0.29 \\
\hline & UGC12344 & $\mathrm{J} 230460.0+184212$ & 1895 & 5 & 8 & 12.80 & & & & & \\
\hline 489 & NGC2573B & J230732.8-890659 & 2311 & 24 & 10 & 12.06 & 12 & 9.70 & 0.8 & & 0.75 \\
\hline & NGC2573A & $\mathrm{J} 231227.2-890734$ & 2328 & 75 & 3 & 12.60 & & & & & \\
\hline 490 & NGC7541 & $\mathrm{J} 231443.9+043204$ & 2894 & 8 & 5 & 8.33 & 37 & 11.25 & 0.0 & & 1.11 \\
\hline & NGC7537 & $\mathrm{J} 231434.5+042954$ & 2893 & 5 & 4 & 10.19 & & & & & \\
\hline 491 & NGC7625 & $\mathrm{J} 232030.1+171332$ & 1878 & 8 & 1 & 8.86 & 177 & $10.60 \mid$ & 0.5 & & 0.50 \\
\hline & UGC12549 & $\mathrm{J} 232153$. & 1888 & 5 & 10 & 13.52 & & & & & \\
\hline 492 & ESO536-003 & $\mathrm{J} 232216.0-234204$ & 177 & 75 & 6 & 13.58 & 62 & 8.79 & 627.0 & & 1.73 \\
\hline & $2 \mathrm{MASX} \ldots$ & $\mathrm{J} 232217.9-23$ & 170 & 74 & 8 & 14. & & & & & \\
\hline 493 & NGC7667 & J232423.1-000629 & 2892 & 7 & 9 & 12.22 & 15 & 9.64 & 1.6 & 1.1 & 1.52 \\
\hline
\end{tabular}


Table I: Table. (Contd.)

\begin{tabular}{|c|c|c|c|c|c|c|c|c|c|c|c|}
\hline No. & Name & $\mathrm{J} 2000.0$ & $\mid \begin{array}{l}V_{L G} \\
\mathrm{~km} \mathrm{~s}\end{array}$ & & $\mathrm{~T}$ & $\begin{array}{r}K \\
\mathrm{mag}\end{array}$ & \begin{tabular}{|c|}
$R_{\perp}$ \\
$\mathrm{kpc}$
\end{tabular} & $\begin{array}{r}\log L \\
L_{\odot}\end{array}$ & $\begin{array}{r}\frac{M}{L_{K}} \\
\odot\end{array}$ & $\begin{array}{r}\frac{M^{c}}{L_{K}} \\
\odot\end{array}$ & $\log (I I)$ \\
\hline & UM160e & J232423.4-000514 & 2912 & \begin{tabular}{|c|c|}
6 & -10 \\
\end{tabular} & 10 & 16.15 & \multirow{3}{*}{31} & \multirow{3}{*}{10.83} & \multirow{3}{*}{33.8} & \multirow{5}{*}{32.7} & \multirow{3}{*}{1.42} \\
\hline \multirow[t]{2}{*}{494} & NGC7637 & J232627.7-815442 & 3447 & 55 & 5 & 9.60 & & & & & \\
\hline & ESO012-001.. & J232555.4-815241 & 3195 & 16 & 5 & 12.02 & & & & & \\
\hline \multirow[t]{2}{*}{495} & UGCA439 & $\mathrm{J} 232632.8+181560$ & 1834 & 60 & 9 & 12.78 & \multirow[t]{2}{*}{137} & \multirow{2}{*}{9.01} & \multirow{2}{*}{828.7} & & \multirow[t]{2}{*}{0.50} \\
\hline & EXG... & $\mathrm{J} 232554.1+183245$ & 1762 & 75 & 9 & 15.66 & & & & & \\
\hline \multirow[t]{2}{*}{496} & NGC7676 & $\mathrm{J} 232901.7-594300$ & 3250 & 35 & -3 & 9.39 & \multirow[t]{2}{*}{410} & \multirow[t]{2}{*}{10.88} & \multirow[t]{2}{*}{70.0} & & \multirow[t]{2}{*}{0.31} \\
\hline & ESO148-009 & J232456.9-594720 & 3354 & 74 & 5 & 13.31 & & & & & \\
\hline \multirow[t]{2}{*}{497} & ESO148-017 & J233006.7-592753 & 2995 & 21. & -5 & 9.48 & \multirow[t]{2}{*}{559} & \multirow[t]{2}{*}{10.78} & \multirow[t]{2}{*}{37.3} & & \multirow[t]{2}{*}{0.31} \\
\hline & ESO148-014 & J232729.2-600952 & 3053 & 74 & 3 & 12.61 & & & & & \\
\hline \multirow[t]{2}{*}{498} & NGC7694 & J233315.8-024210 & 2461 & 5 & 10 & 10.77 & \multirow[t]{2}{*}{10} & \multirow[t]{2}{*}{10.14} & \multirow[t]{2}{*}{3.3} & & \multirow[t]{2}{*}{0.01} \\
\hline & NGC7695 & $\mathrm{J} 233315.0-024313$ & 2400 & 75 & 2 & 12.35 & & & & & \\
\hline 499 & ESO291-034 & $\mathrm{J} 233522.2-425332$ & 3186 & 9 & 4 & 11.63 & 351 & 10.12 & 54.5 & & 0.58 \\
\hline & 2MASX. & J233331.0-423441 & 3144 & 74 & 1 & 12.28 & & & & & \\
\hline 500 & NGC7712 & $\mathrm{J} 233551.6+233707$ & 3319 & 5 & 2 & 10.47 & 383 & 10.53 & 4.5 & 3.2 & 1.67 \\
\hline & UGC12675 & $\mathrm{J} 233438.3+231328$ & 3301 & 5 & 4 & 12.00 & & & & & \\
\hline 501 & NGC7714 & J233614.1+020919 & 3000 & 9 & 3 & 9.74 & 24 & 10.66 & 1.0 & 0.4 & 0.95 \\
\hline & NGC7715 & J233622.1+020924 & $2960 \mid$ & 16 & 9 & 12.70 & & & & & \\
\hline 502 & ESO240-010 & J233743.6-473013 & 3143 & $45 \mid$ & -2 & 8.65 & 302 & 11.20 & 14.6 & 11.2 & 0.16 \\
\hline & ESO240-013 & J233927.0-474629 & 3223 & 10 & 3 & 10.63 & & & & & \\
\hline 503 & NGC7721 & $\mathrm{J} 233848.6-063104$ & 2173 & 5 & 5 & 8.67 & 404 & 10.81 & 8.8 & 5.0 & 0.23 \\
\hline & MCG... & $\mathrm{J} 233848.9-054448$ & 2208 & 11 & 9 & 12.60 & & & & & \\
\hline 504 & NGC7731 & $\mathrm{J} 234129.1+034424$ & 3084 & 11 & 1 & 10.62 & 19 & 10.59 & 0.2 & & 0.55 \\
\hline & NGC7732 & $\mathrm{J} 234133.9+034330$ & 3104 & 17 & 7 & 10.77 & & & & & \\
\hline 505 & NGC7741 & $\mathrm{J} 234354.4+260432$ & 1017 & 5 & 6 & 9.61 & 181 & 9.77 & 0.1 & & 0.31 \\
\hline & UGC12732 & $\mathrm{J} 234039.9+261411$ & 1016 & 5 & 8 & 13.35 & & & & & \\
\hline 506 & NGC7751 & $\mathrm{J} 234658.3+065142$ & 3456 & 9 & 0 & 9.60 & 25 & 10.80 & 18.4 & 18.3 & 1.48 \\
\hline & ESDO... & $\mathrm{J} 234650.7+\mathrm{C}$ & 3259 & 6 & 10 & 14.13 & & & & & \\
\hline 507 & NGC7755 & J234751.8-303119 & 3001 & 7 & 0 & 8.96 & 285 & 10.95 & 21.4 & 7.7 & 0.78 \\
\hline & APMUKS... & $\mathrm{J} 234650.7-301105$ & 2926 & $30 \mid$ & 9 & 15.63 & & & & & \\
\hline 508 & ESO472-006 & $\mathrm{J} 235404.5-252716$ & 3091 & 40 & 9 & 11.74 & 102 & 10.01 & 0.4 & & 0.37 \\
\hline & APMBGC.. & J235440.2-252919 & 3085 & $26 \mid$ & 5 & 12.77 & & & & & \\
\hline 509 & NGC7793 & J235749.8-323528 & 247 & 10 & 7 & 6.85 & 165 & 9.59 & 29.6 & 5.7 & 0.53 \\
\hline & ESO349-031 & J000813.4-343442 & 223 & 11 & 10 & 13.41 & & & & & \\
\hline
\end{tabular}




\section{Acknowledgments}

This work was supported by the Russian Foundation for Basic Research (grant nos. 07-02-00005, 08-02-00627, and 07-02-00792) and the joint grant (no. 06-02-04017) of Deutsche Forschungsgemeinschaft and Russian Foundation for Basic Research. We acknowledge the use of the HyperLeda database (http://leda.univ-lyon1.fr) and NASA/IPAC Extragalactic Database (NED) (http://nedwww.ipac.caltech.edu/). We thank V. E. Karachentseva for finding the magnitudes and types for a large number of dwarf galaxies.
[1] I. D. Karachentsev, V. E. Karachentseva, W. K. Huchtmeier, and D. I. Makarov, Astronom. J. 127, 2031 (2004).

[2] I. D. Karachentsev, Astronom. J. 129, 178 (2005).

[3] I. D. Karachentsev, V. E. Karachentseva, W. K. Huchtmeier, et al., in Proceedings of the Galaxies in the Local Volume, Sydney, 200\%, Ed. by B. Koribalsky and H. Jerden (2008).

[4] R. B. Tully, Astrophys. J. 321, 280 (1987).

[5] R. B. Tully, Nearby Galaxy Catalog (Cambridge Univ. Press, 1988).

[6] R. B. Tully and J. R. Fisher, Astronom. and Astrophys. 54, 661 (1977).

[7] R. B. Tully, E. J. Shaya, I. D. Karachentsev, et al., Astrophys. J. 676, 184 (2008).

[8] J. P. Huchra and M. J. Geller, Astrophys. J. 257, 423 (1982).

[9] A. C. Crook, J. P. Huchra, N. Martimbeau, et al., Astrophys. J. 655, 790 (2007).

[10] J. Vennik, Tartu Astron. Obs. Publ. 73, 1 (1984).

[11] I.D. Karachentsev, Astron. and Astrophys. Trans. 6, 1 (1994).

[12] A. Sandage, Astrophys. J. 307, 1 (1986).

[13] E. F. Bell, D. H. McIntosh, N. Katz, and M. D. Weinberg, Astrophys. J. Suppl. 149, 289 (2003).

[14] M. Fukujita, T. Ichikawa, J. Gunn, et al., Astronom. J. 111, 1748 (1996).

[15] I. D. Karachentsev and A. M. Kut'kin, Pis'ma Astronom. Zh. 31, 299 (2005).

[16] I. Broelis, Dark and Visible Matter in Spiral Galaxies, PhD Thesis (Groningem, 1992).
[17] R. A. Swaters, T. S. van Albada, J. M. van der Hulst, and R. Sancisi, Astronom. and Astrophys. 390, 829 (2002).

[18] A. Begum, J. N. Chengalur, and I. D. Karachentsev, Astronom. and Astrophys. 433, 1 (2005).

[19] J. Guzik and U. Seljak, Monthly Notices Roy. Astronom. Soc. 335, 311 (2002).

[20] G. Paturel, C. Petit, P. Prugniel, et al., Astronom. and Astrophys. 412, 45 (2003)

[21] I. D. Karachentsev, D. I. Makarov, V. E. Karachentseva, and O. V. Mel'nyk, Pis'ma Astronom. Zh. (2008) (in press).

[22] G. de Vaucouleurs, A. de Vaucouleurs, and H. Corwin, Second Reference Catalogue of Bright Galaxies (Texas, Austin, 1976).

[23] T. N. Jarrett, T. Chester, R. Cutri, et al., Astronom. J. 119, 2498 (2000).

[24] T. N. Jarrett, T. Chester, R. Cutri, et al., Astronom. J. 125, 525 (2003).

[25] A. Bizzoni, Monthly Notices Roy. Astronom. Soc. 361, 725 (2005).

[26] M. Fukujita, K. Shimasaku, and T. Ichikawa, Publ. Astronom. Soc. Pacific 107, 945 (1995).

[27] V. E. Karachentseva and I. D. Karachentsev, Astronom. and Astrophys. Suppl. Ser.127, 409 (1998).

[28] V. E. Karachentseva and I. D. Karachentsev, Astronom. and Astrophys. Suppl. Ser.146, 359 (2000).

[29] D. J. Schlegel, D. P. Finkbeiner, and M. Davis, Astrophys. J. 500, 525 (1998).

[30] J. Heisler, S. Tremaine, and J. N. Bahcall, Astrophys. J. 298, 8 (1985). 
[31] J. Binney and M. Merrifield, Galactic astronomy, (Princeton University Press, Princeton, 1998).

[32] A. P. Magtesian, Astrofizika 28, 150 (1988).

[33] E. Gourgoulhon, P. Chamaraux, and P. Fouque, Astronom. and Astrophys. Suppl. Ser. 225, 69 (1992).

[34] H. Arp, Astrophys. J. 256, 54 (1982).

[35] J. N. Chengalur, S. A. Pustilnik, J. M. Martin, and A. Yu. Kniazev, Monthly Notices Roy. Astronom. Soc. 371, 1849 (2006).
[36] B. B. Tully, L. Rizzi, A. E. Dolphin, et al., Astrophys. J. 132, 729 (2006).

[37] I. D. Karachentsev, Binary Galaxies (Nauka, Moscow, 1987) [in Russian].

[38] L. Reduzzi and R. Rampazzo, Astrophys. Lett. and Communic. 30, 1 (1995).

[39] Ekta, J. N. Chengalur, and S. A. Pustilnik, Monthly Notices Roy. Astronom. Soc. 372, 853 (2006).

[40] K. Bekki, astro-ph/0805.0662 